\title{
NMNAT2 is the major NAD+ provider for vesicular glycolysis generating on-board energy for fast axonal transport cargos
}

Subtitle: NMNAT2 meets the specialized metabolic requirements of distal axons

Sen Yang ${ }^{1-3}$, Zhen-Xian Niou ${ }^{1-3}$, Jacob LaMar ${ }^{1,2,4, \S}$, Andrea Enriquez ${ }^{1,3}$, Jui-Yen Huang ${ }^{1-2}$, Jason M. Tennessen ${ }^{5}$, Vidhya Rangaraju ${ }^{4}$, Hui-Chen $\mathrm{Lu}^{1-3^{*}}$

${ }^{1}$ The Linda and Jack Gill Center for Biomolecular Sciences, Indiana University, Bloomington, IN 47405.

2Department of Psychological and Brain Sciences, Indiana University, Bloomington, IN 47405. ${ }^{3}$ Program in Neuroscience, Indiana University, Bloomington, IN 47405.

${ }^{4}$ Max Planck Florida Institute for Neuroscience, Jupiter, FL, USA.

${ }^{5}$ Department of Biology, Indiana University, Bloomington, IN 47405, USA.

$\S$ Current address: Department of Biomedical Science, Florida Atlantic University, Jupiter, FL 33458

*Corresponding author hclu@indiana.edu. 


\section{Abstract}

Axonal transport, an ATP demanding process, plays a critical role in maintaining axonal and neuronal health. ATP in neurons is synthesized by glycolysis and mitochondrial respiration, both driven by proper $\mathrm{NAD}^{+} / \mathrm{NADH}$ redox potentials. NMNAT2 is the major neuronal $\mathrm{NAD}^{+}$ synthesizing enzyme and is a key axonal maintenance factor. Here, we show that NMNAT2 comigrates with fast vesicular cargos in axons and is required for fast axonal transport in the distal axons of cortical neurons. Using SoNar sensor imaging to detect axonal NAD+ and NADH, we show that NMNAT2 is critical in maintaining NAD+/NADH potentials in distal axons. With SynATP sensor imaging to detect synaptic vesicle ATP levels (sv-ATP), we demonstrate that glycolysis is the major provider of sv-ATP and NMNAT2 deletion significantly reduces sv-ATP levels. NAD+ supplementation of NMNAT2 KO neurons restores sv-ATP levels and fast axonal transports in a glycolysis-dependent manner. Together, these data show that NMNAT2 maintains the local NAD+/NADH redox potential and sustains "on-board" glycolysis to meet the bioenergetic demands of fast vesicular transport in distal axons. Intriguingly, mitochondrial respiration contributes significantly to sv-ATP levels in NMNAT2 KO axons. This finding suggests that NMNAT2 deletion induces metabolic plasticity by engaging mitochondria. Surprisingly, NMN, the substrate for NMNAT2 in NAD+ synthesis, supplementation restores svATP and axonal transport in NMNAT2 KO axons with an efficacy similar to NAD+. NMN's restoration of NMNAT2 KO distal axonal sv-ATP levels and vesicle transport requires mitochondrial respiration, while rescue by $\mathrm{NAD}^{+}$is independent of mitochondrial respiration. Based on these findings, we hypothesize that NMN rescues glycolysis to restore axonal transport in NMNAT2 KO axons by taking advantage of the compensatory ATP-generating metabolic pathways triggered by NMNAT2 loss.

\section{Introduction}

Neurons in the mammalian central nervous system often possess extensively arborized axonal processes that navigate over long distances to reach their targets (1). These complex axonal projections are a fundamental unit of brain circuits, where they direct information flow underlying behaviors and cognitive function. Active and well supported axonal transport of specific cargos between cell bodies and axonal terminals is critical in supporting neuronal function and survival (2) as well as retrograde adaptive signaling (3), synaptic assembly and neurotransmission (4). However, the elevated bioenergetic demands, associated with transport along these highly arborized axons, pose huge challenges and vulnerabilities in supplying the necessary energy and maintaining structural integrity of the axons (5-7). Indeed, axonopathy is often an early sign 
of neurodegenerative conditions $(8,9)$. The disruption of axonal transport has been proposed as the primary cause of axonopathy in various neurodegenerative pathologies (10).

The recent discovery that nicotinamide mononucleotide adenylyl transferase 2 (NMNAT2) is a key axonal maintenance factor is exciting as it offers the opportunity to elucidate the mechanisms of active axonal maintenance and to develop novel therapies to prevent axonopathy (11-13). Among three NMNATs (NMNAT1-3), nicotinamide adenine dinucleotide (NAD) synthesizing enzymes, NMNAT2 is the most abundant NMNAT in mammalian brain (1416). NMNAT2 is primarily expressed in neurons and its expression is dramatically decreased in Alzheimer's disease (AD) (17-19), Parkinson's disease (PD) (18, 19), Huntington's disease (HD) (19) and in the spinal cord of amyotrophic lateral sclerosis (ALS) patients (20). NMNAT2 is particularly vulnerable to external insults due to its short half-life (21) and its abundance is quickly reduced following axonal injury, mitochondrial insults or toxin exposure $(21,22)$. Importantly, the upregulation of NMNAT2 and other NMNATs is potently protective across multiple neurodegenerative disease models $(19,23)$. However, the molecular mechanism(s) underlying NMNAT2's axonal maintenance function remains unclear.

NAD is a vital molecule in all organisms, where it is critical for energy homeostasis and is involved in many pathways necessary for health and longevity $(24,25)$. NAD redox potential is constituted by the ratio between the oxidized (NAD+) and reduced (NADH) forms of NAD. Glucose metabolism is the major bioenergetic source in brain, which oxidizes glucose through glycolysis in the cytoplasm and oxidative phosphorylation (OXPHO) in mitochondria to generate ATP. The chemical reactions of glucose metabolism rely on the NAD redox potential, which is maintained by $\mathrm{NAD}^{+}$biosynthesis, consumption/degradation as well as recycling between glycolysis and $\mathrm{OXPHO}(24,25)$. Recent $\mathrm{NAD}^{+}$biosensor imaging studies reveal differential compartmentalization of $\mathrm{NAD}^{+}$in mammalian cells (26). It remains to be determined how NAD redox potential is maintained in the elaborate axons of neurons. Milde et al. showed that NMNAT2 is localized to Golgi-derived vesicles and synaptic vesicles in axons through palmitoylation $(27,28)$ and undergoes fast, bi-directional axonal transport in superior cervical ganglia neurons $(27,29)$. Furthermore, recent studies show that fast axonal transport is primarily fueled by glycolysis with the glycolytic machinery associated with fast-moving vesicles (vesicular glycolysis) instead of mitochondrial $\operatorname{OXPHO}(30,31)$. In this study, we aimed to test the hypothesis that NMNAT2 is localized to fast-moving vesicles in axons to maintain NAD redox potential by local $\mathrm{NAD}^{+}$synthesis and drives "on-board" glycolysis. We found that 
NMNAT2 in cortical glutamatergic neurons is required for fast axonal vesicular transport occurring more than $400 \mu \mathrm{m}$ away from somata. Using NAD+/NADH and Syn-ATP sensors, we showed that deleting NMNAT2 reduces the NAD redox potential in distal axons, while supplementing $\mathrm{NAD}^{+}$restores glycolysis and axonal transport. Intriguingly, we found that NMN (a NAD+ precursor) supplementation recovers the fast axonal transport in the absence of NMNAT2, potentially through adaptive metabolic pathways instituted in mitochondria after NMNAT2 loss.

\section{Results}

NMNAT2 loss results in axonal transport deficits of fast-moving vesicular cargos in distal axons of cortical neurons

We have generated cortical glutamatergic NMNAT2 knockout mice (cKO) using NEX-Cre (Fig1A; (32)) and found that these mice exhibited motor behavior deficits similar to those observed in many neurodegenerative models (33-35). Immunostaining evaluation revealed extensive, age-dependent axonopathy, which was particularly pronounced for long range cortical axons transiting the corpus callosum, internal capsule and fimbria (data not shown). Anterograde and retrograde axonal transport play critical roles in neuronal function and survival (2). Axonal transport deficits have been implicated as one of the primary causes of axonopathy (10). Amyloid Precursor Protein (APP) is rapidly and bidirectionally transported in axons (36) and plays a critical role in synaptogenesis, synaptic function/plasticity, etc. (37). Axonal APP accumulation has been found in Alzheimer's disease mouse models (38) and following traumatic brain injury (39) and is an indicator of axonal transport defects (40). To examine whether NMNAT2 deletion results in axonal transport deficits, APP immunostaining was conducted with brains prepared from postnatal day 21 (P21) cKO and littermate control mice. We found striking APP immunoreactivity in cKO brain region where cortical glutamatergic axons transited, including the corpus callosum, hippocampus and striatum, while little APP signal was detected in the corresponding regions of control brains (Fig 1B-C).

Next, we examined whether APP accumulation occurs in the axons of primary cortical neurons prepared from global NMNAT2 KO embryos (41). Interestingly, starting from 8 days-in-vitro (DIV8), APP accumulated significantly more in KO neurites than in control neurites (Fig 1 D\&F). Immunostaining showed that APP accumulates in $\beta$ III-tubulin-labeled intact axons, but not in MAP2-labeled dendrites, of KO neurons (Fig 1E). APP accumulation increases drastically in KO axons during maturation (Fig $1 \mathrm{~F}$ ), as quantified by a semi-automated method illustrated in Fig 1- 
S1C. Neurite growth measurements by immunostaining showed no obvious growth defect in DIV5-14 NMNAT2 KO neurons (Fig 1-S1A-B). To determine if NMNAT2 deletion causes axonal APP accumulation after neurite formation, we employed a Cre-loxP approach to delete NMNAT2 by adding lentiviral vector carrying Cre cDNA (LV-Cre) to neurons cultured from Nmnat2 floxed $\left(\mathrm{Nmnat}^{\mathrm{f} / \mathrm{f}}\right)$ mice at DIV9, as by this stage cultured cortical neurons have developed most of their axons (Fig 1-S2A). In these cultures, NMNAT2 expression was significantly reduced at DIV12 (Fig 1-S2B), 3 days post LV-Cre transduction, and axonal APP accumulation appeared at DIV14 2 days later (Fig 1-S2C). In addition, SNAP25, synapsin, and PSD95 abundance were also significantly less in KO than controls at DIV14 (Fig 1-S2D), the stage when synaptogenesis normally occurs. These data suggest that NMNAT2 has a direct role in maintaining axonal health and synaptogenesis.

To determine the requirement of NMNAT2 for axonal transport, we used time-lapse imaging to quantify axonal transport of EGFP-tagged vesicular cargos, APP and SNAP25, and DsRedtagged mitochondrial cargos, in axons from control and NMNAT2 KO cortical neurons at DIV4, 6, and 8 (Fig 2 and Fig 2-S1). APP is a component of Rab5 containing vesicles (42) while SNAP25 is a component of Piccolo-bassoon transporting vesicles (43). Both cargos undergo fast, bidirectional axonal transport $(44,45)$ mediated by kinesin- 1 and dynein motor proteins $(36$, 46). Distinct from vesicular cargos, axonal mitochondria are known to intermittently move in both directions at a slower velocity, mediated by the interplay between adaptor and motor proteins $(47,48)$. The axonal transport of these cargos was evaluated in both distal ( $>400 \mu \mathrm{m}$ away from the somata) and proximal axons (within $200 \mu \mathrm{m}$ of the somata) (Fig 2A, Fig 2-S1A). From DIV4 to 6, the transport of APP or SNAP25 cargos in distal axon is unaffected by NMNAT2 loss (Fig 2 C, D, F\&G). However, by DIV8, a defect in APP and SNAP25 transport emerges in KO distal axons (Fig 2B,E), reflected by the following: (i) a remarkable increase in the percentage of stationary/dynamic pause events; (ii) a decrease in the percentage of anterograde moving events (Fig 2C\&F), and (iii) a decrease in anterograde and retrograde velocity (Fig 2D\&G). The transport of APP and SNAP25 was also examined in proximal axons at DIV8, but no obvious alteration was found in KO neurons (Fig 2-S1). In contrast to vesicular transport, quantitative analysis of DsRed-tagged mitochondria revealed comparable mitochondrial distribution and morphology in distal axons of control and KO neurons at DIV8 (Fig 2-S2A-C). There was also no difference in mitochondrial motility between control and $\mathrm{KO}$ axons (Fig 2-S2D-F), despite the heterogenous mitochondrial velocities reported previously (49). Taken together, these results demonstrate that NMNAT2 is required to ensure the transport of vesicular cargos, but not 
mitochondria, and NMNAT2 loss triggers a distal axon transport defect that precedes axon disintegration.

\section{NMNAT2 is required to maintain global NAD ${ }^{+}$and NADH levels and NAD+/NADH redox potential in distal axons}

NMNAT2 is an NAD synthesizing enzyme in the Salvage Pathway (15). The ratio of oxidized $\left(\mathrm{NAD}^{+}\right)$and reduced (NADH) forms of NAD establishes the NAD redox potential (NAD+/NADH), which is crucial to drive energy metabolism $(50,51)$. To determine if NMNAT2 is required for maintaining the $\mathrm{NAD}^{+} / \mathrm{NADH}$ redox potential, we conducted a NAD+/NADH biochemical cycling assay to measure the abundance of $\mathrm{NAD}^{+}$and NADH in DIV8 cortical neurons. We chose DIV8 as the axonal transport deficit and APP accumulation become evident at this stage in NMNAT2 $\mathrm{KO}$ axons (Figs1-2). While we detected a $\sim 50 \%$ reduction in $\mathrm{NAD}^{+}$and $\mathrm{NADH}$ levels in $\mathrm{KO}$ cortical neurons, the net ratios of $\mathrm{NAD}^{+} / \mathrm{NADH}$ remained unchanged between control and $\mathrm{KO}$ neurons (Fig 3A). However, this biochemical assay provides only a global view of the $\mathrm{NAD}^{+} / \mathrm{NADH}$ redox potential across the entire neuronal soma, dendrites, and axons, and any subcellular information is lost.

As our time-lapse imaging studies reveal axonal transport deficits in distal but not proximal axons of NMNAT2 KO neurons, we next examined NAD+/NADH redox potential specifically in distal axons. To examine whether $\mathrm{NAD}^{+} / \mathrm{NADH}$ redox ratios are altered in distal axons of NMNAT2 KO neurons, we employed a genetically encoded sensor, SoNar (52), that allows for the detection of cytosolic NAD redox potential in subcellular regions by live cell imaging. We found that the NAD+/NADH redox potential was significantly reduced in distal axons but not in somata and proximal axons of DIV8 KO neurons (Fig 3B-C). Taken together, the biochemistry and imaging data suggest that NMNAT2 contributes strongly to maintaining the overall abundance of $\mathrm{NAD}^{+}$and $\mathrm{NADH}$ throughout the neuron but determines the cytosolic NAD $/ \mathrm{NADH}^{-}$ redox potential exclusively in distal axons.

\section{NMNAT2 is critical for glycolytic activity in presynaptic varicosities}

Glycolysis is driven by the NAD+/NADH redox potential and fuels fast vesicular cargo transport in axons $(30,31)$. Taking together the axonal transport deficits and reduced NAD+/NADH redox potential in distal axons of NMNAT2 KO neurons, we hypothesized that NMNAT2 in distal axons is critical to maintain the $\mathrm{NAD}^{+} / \mathrm{NADH}$ redox potential required to drive glycolysis and support axonal transport. To directly examine if the reduced $\mathrm{NAD}^{+} / \mathrm{NADH}$ potential in distal axons of 
NMNAT2 KO neurons impairs glycolysis, we employed the luminescence-based ratiometric presynaptic ATP sensor (Syn-ATP) to conduct live imaging in the distal axons of control and KO DIV8 cortical neurons (53). This sensor is composed of an optimized luciferase for ATP detection by luminescence and a mCherry fluorescent protein serving as an internal control for expression levels. Syn-ATP sensor is targeted to synaptophysin-enriched presynaptic varicosities. The luminescence to fluorescence ratio $(L / F)$ measured from Syn-ATP is proportional to ATP levels near synaptic vesicles (sv-ATP) (53) (Fig 4A). As the Syn-ATP sensor is $\mathrm{pH}$ sensitive, the $\mathrm{pH}$ influence on Syn-ATP sensor measurements was corrected with $\mathrm{pH}$ measurements as described in Fig 4-S1A-C. NMNAT2 KO neurons exhibited a modest but significant decrease in sv-ATP compared to controls (Fig 4B-C). ATP can be produced by glycolysis and mitochondrial respiration in mammalian neurons (54). To detect the sv-ATP generated exclusively by the glycolytic pathway, we applied oligomycin to block complex $\mathrm{V}$ of mitochondrial electron transport chain and hence inhibit mitochondrial ATP production. We found that oligomycin treatment did not change control sv-ATP levels in control neurons. In contrast, oligomycin addition further reduced sv-ATP levels in KO distal axons compared to control axons and $\mathrm{KO}$ basal measurements (Fig 4B-C). This finding suggest that glycolysis is the major sv-ATP synthesis pathway and supports our hypothesis that NMNAT2 loss impairs the glycolytic pathway and reduces ATP reduction nearby synaptic vesicles.

To test if NAD+ insufficiency in NMNAT2 KO neurons is the cause for the reduced sv-ATP, culture medium was supplemented with $1 \mathrm{mM} \mathrm{NAD+}$ from DIV5 to DIV8. Previous isotope labeling studies showed that primary cultured neurons can take up exogenous $\operatorname{NAD}^{+}(55,56)$. The augmentation of Sv-ATP levels by $\mathrm{NAD}^{+}$supplementation in control axons with or without oligomycin presence (Fig 4-S1D) suggests that increasing $\mathrm{NAD}^{+}$enhances glycolysis to promote ATP synthesis. Excitingly, NAD+ supplementation restored sv-ATP in KO axons to control levels with or without oligomycin (Fig 4B-C). Taken together, these data suggest that NMNAT2 synthesizes NAD+ to drive glycolysis that provides the majority of ATP for synaptic vesicles in control axons. Notably, the significant and substantial sv-ATP reduction in KO axons caused by oligomycin (Fig 4B-C) suggests the presence of compensatory metabolic plasticity in mitochondrial respiration to provide Sv-ATP after NMNAT2 loss. In other words, the glycolysis deficit in $\mathrm{KO}$ neurons results in compensatory enhancement of mitochondria function. Altogether, the above experiments directly demonstrate that NMNAT2 is critical for axonal glycolysis in presynaptic varicosities. 


\section{NMNAT2 drives a global increase in glycolytic capacity in neurons upon enhanced neurotransmission}

To determine whether NMNAT2 deletion affects global energy metabolism, we used a Seahorse analyzer to measure the dynamics of glycolytic activity and mitochondrial respiration in DIV8 control and $\mathrm{KO}$ neurons. KO neurons exhibited normal glycolytic rate and compensatory glycolytic capacity in baseline condition (Fig 5-S1A-B). NMNAT2 deletion also negligibly impacted basal mitochondrial respiration, maximal respiration capacity, respiration for ATP production and proton leak (Fig 5-S1C-D).

Neuronal activity exacerbates neurodegeneration caused by dNMNAT loss in Drosophila (57). Previously, we found that NMNAT2 KO cortical neurons are more susceptible to depolarization induced excitotoxicity (17). Enhanced glutamate transmission by NMDA boosts glucose uptake and glycolysis in a $\mathrm{Ca}^{2+}$-dependent manner (58). We found that NMNAT2 KO neurons are more susceptible to excitotoxicity triggered by massive depolarization (17). Thus, we hypothesized that NMNAT2 is required to support the additional glycolysis induced by enhanced neuronal activity. To test this hypothesis, we induced chemical LTP with $15 \mu \mathrm{M}$ NMDA and $2 \mu \mathrm{M}$ glycine, a concentration that activates synaptic NMDARs without eliciting excitotoxicity (59), to enhance neurotransmission and therefore increase energy demand. We found that the compensatory glycolytic capacity was significantly reduced in $\mathrm{KO}$ neurons upon chemical LTP induction while the basal glycolytic rates remained normal (Fig 5A-B). NAD+ supplementation of KO neurons increased the basal glycolytic rate and restored the compensatory glycolytic capacity during chemical LTP (Fig 5B). This result suggests that the NAD redox potential maintained by NMNAT2 is crucial for compensatory glycolytic capacity in response to elevated neurotransmission. Collectively, these findings reveal the crucial role of NMNAT2 in supporting glycolytic capacity during enhanced neurotransmission in cortical neurons.

\section{$\mathrm{NAD}^{+}$supplementation relies on glycolysis to restore APP transport in the absence of NMNAT2}

Our SoNar and Syn-ATP sensor studies show that NMNAT2 loss reduces NAD+/NADH redox potentials and sv-ATP levels locally in distal axons (Figs 3-4). Moreover, NAD+ supplementation of $\mathrm{KO}$ neurons restores sv-ATP levels in presynaptic varicosities (Fig 4B). If the reduced redox potential is the major cause for fast axonal transport deficit in NMNAT2 KO distal axons, we hypothesized that we should be able to restore axonal transport by NAD+ supplementation. Supporting this hypothesis, APP transport in KO distal axons was largely recovered by NAD+ 
supplementation as reflected by the decrease in the percentage of stationary/dynamic pause events, increase in the percentage of anterograde events, and increase in anterograde and retrograde velocity, compared to vehicle-treated $K O$ and $N^{\prime} D^{+}$treated controls (Fig 6D). Additionally, supplying NAD+ in culture medium from DIV8 to 14 significantly reduced APP accumulation in $\mathrm{KO}$ neurons revealed by immunostaining (Fig 6-S1). Taken together, NAD+ provided by NMNAT2 is required for APP transport in distal axons.

Recent studies show that fast axonal transport of vesicular cargos is mainly fueled by "onboard" glycolysis through vesicle-associated glycolytic enzymes that provide sufficient ATP to enable motor protein movement, instead of relying on ATP generated by oxidative phosphorylation from mitochondria $(30,31)$. Therefore, we quantitatively examined whether glycolysis mediates the rescue effect from NAD+ supplementation on axonal transport in KO distal axons. We acutely suppressed glycolysis by placing neurons in glucose free medium containing 2-deoxyglucose (2DG) to inhibit glycolysis while adding methyl-pyruvate (Methyl-pyr) as an alternative substrate to support oxidative phosphorylation in mitochondria (Fig 7A-B). On the other hand, to inhibit mitochondrial ATP synthesis, oligomycin was included in the presence of glucose as the glycolysis substrate. Following both treatments, we quantitatively compared the percentage of cargos participating in the stationary/dynamic pause, anterograde and retrograde movement events during the imaging time as well as the velocity for both anterograde and retrograde movements. We found that glycolysis inhibition strongly reduced APP transport in control axons, revealed by significantly increased stationary/dynamic pause events and reduced transport velocity (Fig 7B-E). In contrast, APP transport in control axons was minimally affected when oxidative phosphorylation was inhibited by oligomycin treatment, except for a moderate reduction in anterograde velocity and a moderate increase in retrograde event percentage. In KO axons, glycolysis inhibition blocked the NAD+ restoration of APP cargo transport, reflected by both the percentages of transport events and movement velocity. Taken together, these data suggest that $\mathrm{NAD}^{+}$addition restores APP transport in KO axons mainly via glycolysis, and not by enhancing mitochondrial respiration.

\section{Palmitoylation of NMNAT2 is required for optimal APP transport}

Using time-lapse imaging, we found that NMNAT2 comigrates with APP in the axons of cortical neurons (Fig 8-S1A), similar to what has been observed with superior cervical ganglion (SCG) neurons $(27,29)$. The relative frequency distributions of anterograde and retrograde velocity largely overlap between NMNAT2 and APP (Fig 8-S1B). 
Palmitoylation of NMNAT2 enables its membrane association with vesicular cargos $(27,28)$ and also targets NMNAT2 for MAPK-dependent proteostasis (60). Mutating the two major palmitoylation sites on NMNAT2 (C164S/C165S, PM-NMNAT2) results in its dispersion to the cytoplasm and extended half-life $(27,60)$. We therefore wanted to test if the localization of NMNAT2 on vesicular membranes is required to enable axonal transport in distal axons. We examined whether PM-NMNAT2 could restore APP transport in NMNAT2 KO neurons with a similar efficiency as WT-NMNAT2 (normally palmitoylated). Control and KO neurons were transduced with LVs containing copGFP (control vector) and WT-NMNAT2 or PM-NMNAT2 on DIV2 (Fig 8A, Fig 8-S2A) and then examined for APP accumulation by immunostaining at DIV14 (Fig 8A-B). We found a greater increase in APP accumulation in PM-NMNAT2- than WTNMNAT2-expressing KO axons (Fig 8C) despite similar LV transduction rates (Fig 8-S2B). Western blot analysis showed that the abundance of PM-NMNAT2 is significantly higher than WT-NMNAT2 in KO neurons (Fig 8-S2C), as expected from its longer half-life compared to WTNMNAT2 $(27,60)$. Given that eliminating palmitoylation extended NMNAT2's half-life without impacting $\mathrm{NAD}^{+}$synthesis (28), the reduced rescue offered by PM-NMNAT2 suggests that NMNAT2 must be in close proximity to vesicular cargos by membrane association to facilitate axonal transport. Most likely this close association is required to provide the necessary local $\mathrm{NAD}^{+}$to effectively drive glycolysis.

\section{NMN employs mitochondria to restore sv-ATP and axonal transport in NMNAT2 KO cortical neurons}

NMN is the substrate used by NMNAT2 to synthesize $\operatorname{NAD}^{+}(15)$. It has been shown that the increase in NMN/NAD+ ratio upon NMNAT2 loss or nerve injury activates Sarm1 and results in axon degeneration (61). However, in our experiments, we were surprised to find that NMN supplementation of $\mathrm{KO}$ neurons restored APP transport and mitigated APP accumulation in distal axons (Fig 6, Fig 6-S1). Furthermore, we found that sv-ATP levels were restored to control levels by NMN supplementation in KO distal axons, similar to that of NAD+ supplementation (Fig 4B-C). However, contrary to the recovery by $\mathrm{NAD}^{+}$supplementation, the recovery by NMN is dependent on mitochondrial respiration in NMNAT2 KO axons. Oligomycin treatment abolished the SV-ATP recovery following NMN supplementation in KO presynaptic varicosities. Furthermore, oligomycin also impaired NMN's rescue of APP transport in KO distal axons, reflected by an increase in the percentage of stationary/dynamic pause events and decrease in anterograde percentage and velocity (Fig 7C-E). Intriguingly, NMN supplementation 
also requires glycolysis to rescue APP transport in KO axons, reflected by the substantial increase in stationary/dynamic pause percentage and the decrease in anterograde percentage and velocity after 2DG treatment in NMN-supplemented $\mathrm{KO}$ axons. Taken together with the enhanced mitochondrial function in KO axons (Fig 4), data from our sv-ATP and axonal transport studies suggest that NMN engages mitochondria to restore glycolysis, that subsequently fuels axonal transport in $\mathrm{KO}$ distal axons.

\section{Discussion}

The discovery of NMNAT2's role in axonal health $(21,62)$ provides a critical entry point into understanding how neuronal function is actively maintained. Here, we reveal mechanistic insights into the NAD synthesis activity of NMNAT2 that enables on-board glycolysis to generate the ATP necessary to fuel fast axonal transport of vesicular cargos. First, we show that NMNAT2 is specifically co-localized with vesicular cargos undergoing fast axonal transport. NMNAT2 loss results in defective antero- and retrograde fast axonal transport of vesicular cargos such as synaptic vesicles, without impacting slow axonal transport of mitochondria. Using specific sensors and pharmacological approaches, we demonstrate that NMNAT2 is necessary for maintaining the NAD+/NADH redox potential that drives the glycolytic ATP synthesis to fuel vesicular cargo transport. NAD ${ }^{+}$supplementation of NMNAT2 KO neurons restores vesicular ATP (sv-ATP) levels and axonal transport. Blocking glycolysis abolishes $\mathrm{NAD}^{+}$-dependent recovery of NMNAT2 KO axonal phenotypes. Interestingly, the impact of NMNAT2 deletion on NAD+/NADH redox potentials, glycolysis, ATP levels and axonal transport is confined to distal axons, sparing somata and proximal axons. Furthermore, at the whole neuronal level, the impact of NMNAT2 loss on glycolysis is only evident after enhanced glutamate transmission with no impact on mitochondrial respiration. In wildtype axons, the glycolytic pathway provides the majority of ATP for synaptic vesicle transport $(30,31)$. In contrast, mitochondrial respiration is required for maintaining sv-ATP levels in NMNAT2 KO distal axons. This finding suggests that NMNAT2 deletion induces metabolic plasticity by activating mitochondria to maintain sv-ATP levels. Surprisingly, NMN supplementation can restore axonal sv-ATP levels and transport in NMNAT2 KO axons with an efficacy similar to NAD'. However, NMN's restoration of NMNAT2 KO distal axonal sv-ATP levels and vesicle transport requires mitochondrial respiration while rescue by $\mathrm{NAD}^{+}$is independent of mitochondrial function. Moreover, the recovery of the axonal transport deficit in NMNAT2 KO axons by NMN depends on both glycolysis and mitochondria respiration. Taken together, we hypothesize that NMN rescues glycolysis to restore axonal transport in NMNAT2 KO axons by 
taking advantage of the compensatory ATP-generating metabolic pathways triggered by NMNAT2 loss. NMNAT2 is significantly reduced in the brains of patients with AD, PD, and several neurodegenerative conditions (17-20). Interestingly, reduced glucose metabolism has also been observed in the brains of patients with neurodegeneration (63). Our findings suggest the importance of elucidating the metabolic plasticity induced by NMNAT2 reduction in patients to develop therapeutic approaches to maintain brain health.

\section{NMNAT2 is required for fast axonal transport to maintain local NAD+/NADH redox potential and drive glycolysis on vesicular cargos in distal axons}

By combining sensor imaging and pharmacology, our study provides several lines of evidence supporting the necessity of NMNAT2 in providing ATP by glycolysis to support fast axonal transport. NMNAT2 loss in cortical glutamatergic neurons reduced NAD+/NADH redox potential and impaired glycolysis in synaptic vesicle-enriched varicosities of distal axons. Glycolysis is normally tightly coupled to mitochondrial respiration, where NAD+ regenerates from mitochondrial OXPHO (64). However, in differentiated neurons with polarized morphologies, mitochondria occupy only $~ 5 \%$ of the axonal length (65) and have relatively slow motility (6668). Distinctively, axonal glycolytic enzymes are associated "on-board" with fast moving vesicles in axons $(30,31,67)$. Thus, in axons, the opportunity for glycolysis to be tightly coupled with mitochondrial OXPHO is greatly reduced due to significant spatiotemporal challenges. Indeed, we find that fast axonal transport of APP-containing cargos mostly depends on glycolysis. Furthermore, NAD+ restores NMNAT2 KO axonal transport deficits independent of mitochondrial respiration. This finding is consistent with previous studies showing that BDNF transport is fueled by glycolysis and is unaffected by ablating mitochondrial respiration (30).

Our developmental studies utilizing time-lapse imaging of axonal transport and immunostaining reveal that DIV8 is the critical time when NMNAT2 becomes indispensable for axonal transport. At DIV8, cortical neurons have acquired the majority of their neurites and begun synaptogenesis (69). Interestingly, DIV8 is also when NMNAT2 expression significantly increases (28), fast axonal transport velocity significantly increases (Fig 2D; (67)), and mitochondrial mobility decreases (66-68) in cortical neurons. Furthermore, we found that NMNAT2 co-localizes with fast transported cargos such as synaptic vesicles and APPcontaining vesicles (Fig 8-S1). Taken together with the reduced velocities of fast axonal transport in DIV8 NMNAT2 KO distal axons, we propose that the increase in NMNAT2 during development augments the velocity of fast axonal transport by providing ample $\mathrm{NAD}^{+}$source to generate ATP via glycolysis to fuel neighboring fast-moving vesicles. 
Glycolysis generates two ATP molecules and reduces two NAD+ to two NADH. Two major routes to regenerate NADH to NAD+ include: (1) the coordinated reduction of pyruvate to lactate by lactate dehydrogenase A (LDH-A); (2) the mitochondrial OXPHO and NADH shuttles $(64,70)$. Maximilian et al. found that LDH-A preferentially localizes to axonal vesicular cargos containing BDNF (71). LDH-A's lactate producing and NAD+ regenerating activities are required for axonal BDNF transport. NMNAT2 is associated with Golgi-derived vesicles through palmitoylation(27). We find that NMNAT2's ability to associate with vesicles is important for it to maximally support fast axonal transport (Fig 8). Specifically, PM-NMNAT2, despite its longer half-life, is less effective than WT-NMNAT2 in restoring axonal transport deficits in NMNAT2 KO axons. It has been estimated that kinesin requires $~ 187.5$ ATP molecules/s to move cargos undergoing fast axonal transport at $\sim 1.5 \mu \mathrm{m} / \mathrm{s}$ (72). Thus, assuming glycolysis as the sole energy generation pathway, 94 cycles of glycolysis per second will be required to generate the required ATP. The close proximity of NMNAT2 to the vesicular glycolytic complex is likely to be critical to sustain the efficacy of vesicular glycolysis. Taken together, we postulate that NMNAT2 and $\mathrm{LDH}-\mathrm{A}$ act in concert in maintaining the proper $\mathrm{NAD}+\mathrm{NADH}$ redox potential to boost the efficacy of on-board glycolysis and support fast axonal transport.

A recent report shows that prolonged $\mathrm{ZDHHC17}$ loss, the palmitoyl acyltransferase that palmitoylates NMNAT2, leads to distal axon degeneration in dorsal root ganglia and optic nerve, and can be rescued by NAD+ supplementation (73). The current study sheds light on the importance of palmitoylation-dependent vesicular localization of NMNAT2 in its function. Promisingly, a recent study shows that pharmacologically enhancing brain palmitoylation restores BDNF axonal transport in vitro and recovers pathological and behavioral deficit in vivo in a Huntington's disease model (74). Whether the success of this neuroprotective treatment requires palmitoylated NMNAT2 merits further investigation.

The live neuron imaging experiments delineate the selective bioenergetic vulnerability of distal axons following NMNAT2 loss. Interestingly, proximal axons (<200 $\mu \mathrm{m}$ from the soma) show normal transport of APP and SNAP25 and exhibit a normal NAD+/NADH redox potential in NMNAT2 KO neurons at DIV8, while the distal axonal deficiency has already developed. Data from the biochemical assay highlight the contribution of NMNAT2 in maintaining total NAD (NAD ${ }^{+}$and $\mathrm{NADH}$ ) abundance in cortical neurons. However, the global NAD+/NADH redox potential is not altered by NMNAT2 loss. Cambronne et al. showed that the NAD+ pool between nucleus and cytoplasm are interchangeable in mammalian cells (26). Analysis of RNA-seq transcriptomic data reveals that the NAD salvage pathway is the major NAD synthesis pathway in cortical neurons and NMNAT1 and NMNAT2 are the major (if not the only) NAD synthesizing 
enzymes (data not shown). In NMNAT2 KO cortical neurons, the axonal regions proximal to soma could potentially receive $\mathrm{NAD}^{+}$generated by nuclear NMNAT1 and thus maintain an adequate redox potential to drive axonal transport. Recent electron microscopy reconstructions of layer $2 / 3$ pyramidal neurons in the primary visual cortex reveals higher mitochondrial coverage and a larger mitochondrial volume in axonal regions within $100 \mu \mathrm{m}$ of the soma (75). Thus, it is plausible that mitochondria located in proximal axons provide sufficient ATP to enable fast axonal transport despite the absence of NMNAT2. In contrast, distal axons possess fewer, smaller and less mobile mitochondria $(66-68,75)$. Distal axons are also too far to receive the $\mathrm{NAD}^{+}$generated by NMNAT1. NMNAT3 expression is not detectable in cortical neurons (data not shown) and thus NMNAT2 is likely to be the sole NAD+ synthesizing enzyme in distal axons. In addition, the need for $\mathrm{NAD}^{+}$in aerobic glycolysis becomes more evident upon maturation or stimulation (76, 77), further increasing the vulnerability of distal axons following NMNAT2 loss. Interestingly, when challenged by enhanced neuronal activity, the defect in compensatory glycolytic capacity becomes evident in NMNAT2 KO neurons that can be alleviated by NAD+ supplementation. Overall, our findings provide genetic and bioenergetic insights into understanding the vulnerability of long-range connectivity of brain circuits.

\section{Exogenous NMN supplementation restores NMNAT2 loss-of-function by inducing mitochondrial ATP synthesis near synaptic vesicles and rescues distal axonal transport}

The rescue of vesicular ATP levels and axonal transport in NMNAT2 KO axons by NMN supplementation was unexpected. Compelling evidence show that an increase in NMN/NAD+ ratio after NMNAT2 loss activates the metabolic sensor Sarm1 to trigger axon degeneration (61). We too confirmed that deleting Sarm1 rescues the axonopathy in NEX-NMNAT2 cKO brains and eliminates the axonal APP accumulation (data not shown). However, we found that NMN supplementation did not affect axonal transport of APP cargos; while FK866 treatment, the NAMPT inhibitor that blocks NMN synthesis, resulted in APP accumulation in control neurons (data not shown).

Our biochemical assay shows that NMN supplementation does not alter global NAD+ abundance. In contrast, NAD+ supplementation increases NAD+ levels within 1 hour of treatment (data not shown). Using the Syn-ATP sensor, we found that NMN restored SV-ATP levels and this rescue depends on mitochondrial respiration. Intriguingly, NMN's rescue of APP axonal transport relies on both glycolysis and mitochondrial respiration. We postulate that NMN rescues NMNAT2 KO axonal function by enabling mitochondria to support on-board vesicular 
glycolysis. Different from control axons, inhibiting mitochondrial respiration in NMNAT2 KO distal axons greatly reduced sv-ATP levels. This suggests a remodeling of metabolic pathways to compensate for glycolysis deficits in NMNAT2 KO axons. Interestingly, RNA-seq revealed that the top pathways upregulated in NMNAT2 KO neurons is mitochondrial metabolism, including TCA cycle and OXPHO (data not shown). Our RNA-seq study also reveals the upregulation of genes in the malate-aspartate shuttle, glycerol phosphate shuttle and mitochondrial NAD+ transporter in NMNAT2 KO neurons. We also found that NMNAT2 loss does not alter global mitochondrial respiration and has no impact on mitochondrial density, morphology, or motility in distal axons. It remains to be determined whether mitochondria synthesize more ATP directly or export NAD+ to support vesicular glycolysis in NMNAT2 KO distal axons (58).

In the NMN rescued condition, whether mitochondria act upstream of glycolysis in distal axons by exporting more $\mathrm{NAD}^{+}$into the cytosolic pool also warrants further investigation. The beneficial impacts offered by NMN administration have been demonstrated across diverse aging and disease models (78). Our findings suggest that NMN's therapeutic potential may not require NMNAT2, which could be useful in advanced neurodegenerative conditions where NMNAT2 is reduced. How NMN is metabolized and utilized by mitochondria in NMNAT2 KO neurons also demands further investigation. Taken together, our study implicates the importance to elucidating metabolic plasticity under pathological conditions and identify novel therapeutic agents to sustain energy metabolism.

\section{Acknowledgements:}

This work is supported by NIH-NINDS NS086794 to H.C.L, NIH-NIGMS R35GM119557 to J.M.T. and the Indiana Clinical and Translational Sciences Institute funded by NIH UL1TR002529. V.R. is funded by the Max Planck Society. The content is solely the responsibility of the authors and does not necessarily represent the official views of $\mathrm{NIH}$. We thank the following: Dr. Yuyu Song for helpful discussions; Light Microscopy Imaging Center and Dr. Jim Powers in Indiana University Bloomington for live cell imaging (NIH1S10OD024988); Gene vector core directed by Dr. Kazu Oka at Baylor College of Medicine for lentivirus production; Drs. Jonathan Gilley and Michael Coleman for pC1-EGFP-SNAP25 plasmid, pN1-mCherry-NMNAT2 plasmid and NMNAT2 ${ }^{\mathrm{f} / \mathrm{f}}$ mice; Dr. Yi Yang for providing SoNar sensor; Drs. Feng Guo, István Katona for sharing equipment; Dr. Ken Mackie for pCAGmCherry plasmid and reading the manuscript; László Barna, John Reinhart, Scott Barton. Theo 
Knowles, Caliel D. Hines and Kelsie Godsey for technical assistance; Dr. JangDong Seo in Indiana statistical consulting center for statistical assistance.

\section{Author Contributions:}

Conceptualization: S.Y., H.C.L., V.R., J.M.T, JY.H.

Experimentation/Investigation: S.Y., ZX. N., J.L., A.E.

Data acquisition: S.Y., ZX.N., J.L., A.E.

Data curation: S.Y., V.R., ZX.N.

Methodology: V.R., JY.H.

Writing: S.Y., HC.L., V. R.

Funding acquisition: H.C.L.

Resource: H.C.L., V.R.

Supervision: H.C.L., V.R. 


\section{Figure Legends}

\section{Fig 1. Loss of NMNAT2 triggers APP accumulation in axons}

(A) Illustration of Nmnat2 conditional KO (cKO) mice generation as described previously (79). The dark and light green triangles indicate loxP sites. (B) Representative APP and DAPI staining images of the corpus callosum (CC), hippocampus (Hipp) and striatum (Str) from brains of postnatal 21 day-old control (ctrl) and cKO mice. Scale bar $=150 \mu \mathrm{m}$. (C) Bar graphs summarizing APP signals in $\mathrm{CC}(\mathrm{N}$, animal numbers; ctrl, $\mathrm{N}=6$; cKO $\mathrm{N}=4$; Mann-Whitney test), Hipp (ctrl, $\mathrm{N}=3$; cKO, $\mathrm{N}=3$; unpaired t-test) and $\mathrm{Str}$ (ctrl, $\mathrm{N}=3$; cKO, $\mathrm{N}=3$; unpaired t test). (D) Representative APP (black) immunostaining images taken in DIV5, DIV8, DIV10 and DIV14 ctrl and $\mathrm{KO}$ cortical neurons. Scale bar $=30 \mu \mathrm{m}$. Arrowheads indicate accumulated APP. (E) Images showing APP signal in TUJ1 (axonal marker) labeled axons but not in MAP2 (dendritic marker) labeled dendrite in KO DIV8 neurons. Scale bar $=5 \mu \mathrm{m}$. (F) Summaries of normalized areas of APP accumulation in neurites relative to ctrl. Images used for analysis (n): DIV5: ctrl, n=10; KO, n=9; DIV8 \& DIV10: ctrl, $n=20 ; K O, n=20 ;$ DIV14: ctrl, $n=50 ; K O, n=67$. DIV5-10 data were collected from 2 independent culture experiments, while DIV14 data came from $>3$. KO was compared to ctrl at the same DIV. Statistics were conducted with Multiple Mann-Whitney tests with Holm-Šídák post-hoc analysis. ${ }^{* *}, \mathrm{p}<0.01 .{ }^{* * *}, \mathrm{p}<0.001,{ }^{* \star * *}, \mathrm{p}<0.0001$, for this and subsequent figures. The mean, SEM, N and statistical detail are provided in Supplement Table1 for this and subsequent figures.

\section{Fig 1-S1. Gross neurite growth is not affected by NMNAT2 loss}

(A) Representative images of MAP2 and $\beta$ III-tubulin staining in ctrl and KO neuronal cultures at DIV8. Soma and dendritic regions (labeled by MAP2) were subtracted from $\beta$ III-tubulin staining images to determine axon-corresponding regions. Scale bar $=100 \mu \mathrm{m}$. (B) Summaries for somatic and dendritic area labeled by MAP2 and, axonal area labeled by $\beta$ III-tubulin (MAP2 area subtracted), in ctrl and KO neuronal cultures at DIV5, 8, 10 and 14. KOs were compared to ctrls at the same DIV. (C) Semi-automated procedure used to quantify APP accumulation by ImageJ.

\section{Fig 1-S2. NMNAT2 loss after axonal outgrowth leads to APP accumulation and synaptic} protein reduction

(A) Timeline of lentivirus administration and sample collection. (B) NMNAT2 mRNA levels relative to copGFP ctrl in DIV12 cortical neurons. (C) Area of accumulated APP relative to copGFP ctrl in DIV14 cortical neurons. (D) Representative western blots of SNAP25, Synapsin, 
PSD95 and GAPDH (loading control) in DIV14 cortical neurons and the corresponding quantification.

\section{Fig 2. NMNAT2 is required for transporting vesicular cargos in distal axons}

(A) I, Diagram indicating the regions corresponding to distal axons (> $400 \mu \mathrm{m}$ away from the soma) where axonal transport was examined with time-lapse imaging. II, Example kymograph (left) and annotations (middle) with anterograde (red lines), retrograde (yellow lines) and stationary/dynamic pause movements (white lines). Snapshots showing APP-GFP movement along an axon segment at 39, 41, 43 and $45 \mathrm{~s}$. Scale bar $=10 \mu \mathrm{m}$. (B, E) Representative kymographs for APP-EGFP and SNAP25-EGFP from DIV8 WT and KO distal axons. Scale bar $=20 \mu \mathrm{m}$. (C, F) Average percentage of APP- or SNAP25-EGFP vesicles exhibiting anterograde, retrograde and stationary/dynamic pause movements from 2 independent culture experiments. DIV, $\mathrm{n}$ (axon segment from individual neuronal condition); and statistics: (C) DIV4, $24 \mathrm{ctrl}$ and $28 \mathrm{KO}$; DIV6, $22 \mathrm{ctrl}$ and $19 \mathrm{KO}$; DIV8, $23 \mathrm{ctrl}$ and $30 \mathrm{KO}$; compared with two-way ANOVA and Tukey's multiple comparisons. (F) DIV6, 23 ctrl, 17 KO; DIV8, 23 ctrl, 19 KO; two-way ANOVA with Tukey's multiple comparisons. (D, G) Summaries for the velocity of antero/retrograde transport from 2 independent culture experiments. DIV, n (axon segments from individual neuronal conditions); and statistics: (D) Anterograde velocity analysis: DIV4, 23 ctrl and $28 \mathrm{KO}$; DIV6, $21 \mathrm{ctrl}$ and $16 \mathrm{KO}$; DIV8, 23 ctrl and $26 \mathrm{KO}$; two-way ANOVA with Tukey's multiple comparisons. Retrograde velocity analysis: DIV4: 24 ctrl, 28 KO; DIV6: 22 ctrl, 16 KO; DIV8, 23 ctrl, $28 \mathrm{KO}$; multiple Mann-Whitney test with Holm-Šídák post-hoc analysis. (G) Anterograde velocity analysis: DIV6, 21 ctrl, $17 \mathrm{KO}$; DIV8, $21 \mathrm{ctrl}, 16 \mathrm{KO}$; two-way ANOVA with Tukey's multiple comparisons. Retrograde velocity analysis: DIV6, 22 ctrl, $17 \mathrm{KO}$; DIV8, 23 ctrl, $17 \mathrm{KO}$; two-way ANOVA with Tukey's multiple comparisons test. KO was compared to ctrl of the same DIV in all the above data sets.

\section{Fig 2-S1. Vesicular cargo transport in proximal axons is less affected by NMNAT2 loss}

(A) Diagram indicating the regions corresponding to proximal axons (within $200 \mu \mathrm{m}$ from the soma) where axonal transport was examined with time-lapse imaging. (B) Representative kymographs show the mobility of EGFP-tagged APP vesicles in proximal axon (within $200 \mu \mathrm{m}$ of the soma) of DIV8 ctrl and KO neurons. (Scale bar $=20 \mu \mathrm{m}$; total recording time $=60 \mathrm{~s}$ ). (C) Percentage of APP vesicles that exhibit anterograde, retrograde, and stationary/dynamic pause movements in proximal axons of DIV8 ctrl and KO neurons. (ctrl $\mathrm{N}=23, \mathrm{KO} N=27$ ). (D) Velocity of anterogradely and retrogradely moving APP vesicles in proximal axons of DIV8 ctrl and KO 
neurons. (ctrl N=23, KO N=26). (E) Representative kymographs show the mobility of EGFPtagged SNAP25 vesicles in proximal axons of DIV8 ctrl and KO neurons. (Scale bar = $20 \mu \mathrm{m}$; total recording time $=60 \mathrm{~s})$. $(\mathbf{F})$ Average percentage of SNAP25 vesicles that exhibit anterograde, retrograde, and stationary/dynamic pause movements in proximal axons of DIV8 ctrl and $\mathrm{KO}$ neurons. (ctrl $\mathrm{N}=16, \mathrm{KO} \mathrm{N}=14$ ). (G) Velocity of anterogradely and retrogradely moving SNAP25 vesicles in proximal axons of DIV8 ctrl and $\mathrm{KO}$ neurons. (ctrl $\mathrm{N}=16, \mathrm{KO} N=14$ ). $\mathrm{N}$ represents the number of cells imaged from 2 cultures. Two-way ANOVA with Sidak's multiple comparisons test. Data represent mean \pm SEM.

\section{Fig 2-S2. Mitochondrial density, morphology and mobility are unaffected by NMNAT2 loss in distal axons}

(A) Representative images of mCherry and Mito-Venus in distal axon of DIV8 ctrl and KO neuron. (Scale bar $=10 \mu \mathrm{m}$ ). (B) Mitochondria density (\# of mitochondria per $10 \mu \mathrm{m}$ of axon) in distal axons of DIV8 ctrl and $\mathrm{KO}$ neurons (ctrl $\mathrm{N}=28, \mathrm{KO} \mathrm{N}=30$ ). Unpaired t-test was used. (C) The cumulative percentage distribution of mitochondria morphology indicated by sphericity in DIV8 ctrl and $\mathrm{KO}$ distal axons (ctrl $\mathrm{N}=28, \mathrm{KO} N=30)$. $\log (\mathrm{Y} /(1-\mathrm{Y})$ ) transformation for independent variable and linear mixed model with random intercept and slope was used, see descriptions in Material and Methods. (D) Representative kymographs show the mobility of MitoDsRed in distal axons of DIV8 ctrl and KO neurons. (Scale bar $=20 \mu \mathrm{m}$; total recording time $=15 \mathrm{~min}$ ). (E) Velocity of anterogradely and retrogradely moving MitoDsRed in distal axons of DIV8 ctrl and KO neurons (Anterograde: ctrl $\mathrm{N}=14, \mathrm{KO} \mathrm{N}=16$; Retrograde: ctrl $\mathrm{N}=14, \mathrm{KO} N=14$ ). For analyzing anterograde movement, Mann-Whitney test was used; for retrograde movement, unpaired t-test was used. (F) Percentage of MitoDsRed that exhibit anterograde, retrograde, and stationary/dynamic pause movements in distal axons of DIV8 ctrl and KO neurons. (ctrl $\mathrm{N}=19, \mathrm{KO}=21$ ). For analyzing anterograde and stationary/dynamic pause movements, unpaired t-test was used; for retrograde movements, Mann-Whitney test was used. $\mathrm{N}$ represents cell numbers from 2 cultures. Data represents mean \pm SEM.

Fig 3. Loss of NMNAT2 reduces NAD+ levels and impairs the NAD redox potential in distal axons

(A) Summaries of normalized levels of $\mathrm{NAD}^{+}$and $\mathrm{NADH}$ and their corresponding NAD+/NADH ratio in DIV8 ctrl and KO cortical neurons measured by the NAD+/NADH-Glo assay (readings from $28 \mathrm{ctrl}$ and $28 \mathrm{KO}$ culture wells from 3 independent culture experiments; unpaired t-test was used for NADH while Mann-Whitney test was used for both $\mathrm{NAD}^{+}$and NAD ${ }^{+} \mathrm{NADH}$ ratios 
(due to data distributions). (B) Representative images show signals emitted from the SoNar $\left(\mathrm{NAD}^{+} / \mathrm{NADH}\right)$ ratiometric sensor for $\mathrm{NAD}^{+}$and $\mathrm{NADH}$ in the proximal and distal axons from DIV $8 \mathrm{ctrl}$ or KO cortical neurons. Scale bar $=20 \mathrm{um}$. (C) Summaries of the NAD+/NADH ratio in soma, proximal and distal axons of DIV7 \& $8 \mathrm{ctrl}$ and $\mathrm{KO}$ cortical neurons revealed by F488/F440 ratiometric measurements of SoNar (Soma: $25 \mathrm{ctrl}$ and $24 \mathrm{KO}$; proximal axon segments: $17 \mathrm{ctrl}$ and $18 \mathrm{KO}$; distal axon segments: $23 \mathrm{ctrl}$ and $26 \mathrm{KO}$; from 2 independent culture experiments; two-way ANOVA with Tukey's multiple comparisons test).

\section{Fig 4. NMNAT2 loss impairs glycolysis in distal axon presynaptic varicosities}

(A) Schematic diagram of the Syn-ATP sensor and the Syn-ATP imaging workflow. Syn: synaptophysin, mCh: mCherry, Luc: luciferase. (B) $\mathrm{pH}$ corrected L/F ratio proportional to svATP levels measured by the Syn-ATP sensor in mCherry positive varicosities of distal axons of DIV8 ctrl, KO, KO+NAD+ and KO+NMN neurons. Synaptic varicosities from 2-3 batches of cultures imaged under basal conditions: $78 \mathrm{ctrl}, 77 \mathrm{KO}, 48$ for $\mathrm{KO}+\mathrm{NAD}^{+}, 41$ for $\mathrm{KO}+\mathrm{NMN}$. Synaptic varicosities imaged with Oligomycin: $67 \mathrm{ctrl}, 68 \mathrm{KO}, 48 \mathrm{KO}+\mathrm{NAD}^{+}, 41 \mathrm{KO}+\mathrm{NMN}$. Kruskal-Wallis test was used. (C) Representative Syn-ATP luminescence and fluorescence images of distal axon varicosities under basal and Oligo treated conditions from DIV8 ctrl, KO, $\mathrm{KO}+\mathrm{NAD}^{+}$and $\mathrm{KO}+\mathrm{NMN}$ neurons. Scale bar $=5 \mu \mathrm{m}$.

\section{Fig 4-S1. pH measurement and pH correction for Syn-ATP}

(A) Cyto-pHluorin imaging workflow and equation used for cytosolic $\mathrm{pH}$ determination in presynaptic varicosities. (B) Average $\mathrm{pH}$ measured by Cyto-pHluorin in various conditions. The mean of each group is labeled above each box and whisker plot. Basal: ctrl $\mathrm{N}=27, \mathrm{KO} N=25$, $\mathrm{KO}+\mathrm{NAD}+\mathrm{N}=27, \mathrm{KO}+\mathrm{NMN} \mathrm{N}=28$; Oligo: $\operatorname{ctrl} \mathrm{N}=18, \mathrm{KO} N=25, \mathrm{KO}+\mathrm{NAD}+\mathrm{N}=27, \mathrm{KO}+\mathrm{NMN} \mathrm{N}=28$. $\mathrm{N}$ represents number of neurons. Two-way ANOVA with Tukey's multiple comparisons test, ${ }^{*} \mathrm{p}=$ $0.0266,{ }^{* *} \mathrm{p}=0.0042,{ }^{* * * *} \mathrm{p}<0.0001$. (C) Equations used for $\mathrm{pH}$ correction of $\mathrm{L} / \mathrm{F}$ measurements from Syn-ATP. (D) L/F measurements in distal axon varicosities of DIV8 ctrl neurons with and without $\mathrm{NAD}^{+}$and $\mathrm{NMN}$ supplementation. Basal: ctrl $\mathrm{N}=78, \operatorname{ctrl}+\mathrm{NAD}+\mathrm{N}=10, \operatorname{ctrl}+\mathrm{NMN} \mathrm{N}=11$; Oligo: ctrl $\mathrm{N}=67$, ctrl $+\mathrm{NAD}+\mathrm{N}=10$, ctrl$+\mathrm{NMN} \mathrm{N}=11$. $\mathrm{N}$ represents the number of neurons analyzed. Two-way ANOVA with Dunnett's multiple comparisons test, ${ }^{* *} \mathrm{p}=0.0048$, ${ }^{* * * *} p<0.0001$. Data represents mean \pm SEM.

Fig 5. Compensatory glycolytic capacity of NMNAT2 KO neurons is significantly reduced upon chemical LTP induction. 
(A, B) Summarized basal and compensatory glycolysis measured from DIV8 ctrl and KO cortical neurons using the Seahorse XF Glycolytic Rate Assay. (A) Average change in glycolytic proton efflux rates (GlycoPER) with time. The treatment timeline for chemical LTP induction with NMDA \& glycine, mitochondrial respiration inhibition with rotenone \& antimycin and glycolysis inhibition with 2DG are indicated at the top. (B) Summaries for GlycoPER in basal, LTP and compensatory phase in ctrl, $\mathrm{KO}, \mathrm{KO}_{+} \mathrm{NAD}^{+}$and $\mathrm{KO}+\mathrm{NMN}$ conditions at DIV8. As KO neurons were present in all batches of experiments, GlycoPER raw values across all groups were normalized to $\mathrm{KO}$ as percentage. Numbers of culture wells from 2-4 independent culture experiments: $12 \mathrm{ctrl}, 24 \mathrm{KO}, 19 \mathrm{KO}+\mathrm{NAD}^{+}, 18 \mathrm{KO}+\mathrm{NMN}$. Brown-Forsythe and Welch ANOVA with Dunnett's T3 multiple comparisons test was used for basal conditions while the KruskalWallis test was used for NMDA-induced and compensatory phases.

Fig 5-S1. Glycolysis and mitochondrial respiration is unaffected at rest by NMNAT2 loss at DIV8

(A) Representative response of glycolytic proton efflux rate (GlycoPER) over time after sequential addition of Rotenone \& Antimycin and 2DG in DIV8 Ctrl and KO neuronal culture. Data represents mean \pm SEM. (B) Quantification of GlycoPER in basal and compensatory phase in DIV8 Ctrl and KO. GlycoPER raw value is normalized to Ctrl as percentage. (Ctrl $\mathrm{N}=19$, KO $\mathrm{N}=19$. $\mathrm{N}$ represents well number in 24-well Seahorse assay plate from 2 batch of culture.) Two-way ANOVA was used. (C) Representative response of oxygen consumption rate (OCR) over time after sequential addition of Oligomycin, FCCP and Rotenone \& Antimycin in DIV8 Ctrl and KO neuronal culture. Data represents mean \pm SEM. (D) Quantification of OCR in basal phase, portion accounted for ATP production, maximal respiration phase and portion accounted for proton leak in DIV8 Ctrl and KO neuron. OCR raw value is normalized to Ctrl as percentage. (Ctrl $\mathrm{N}=13, \mathrm{KO}=11, \mathrm{~N}$ represents well number in 24-well Seahorse assay plate from 3 batch of culture.) Multiple Mann-Whitney test with Holm-Šídák correction for comparisons was used.

Fig 6. Supplementing with NAD+ or NMN rescues APP transport in NMNAT2 KO neurons (A) Experimental timeline. (B) Representative kymographs from ctrl and KO cultures with vehicle, $1 \mathrm{mM} \mathrm{NAD}^{+}$or $1 \mathrm{mM}$ NMN supplementation from DIV5 till DIV8. Scale bar $=20 \mu \mathrm{m}$. (C, D) Average percentage of APP-EGFP vesicles exhibiting anterograde, retrograde and stationary movements and their velocities in distal axons of DIV8 ctrl and KO neurons in the presence of vehicle, NAD+ or NMN. N and statistics (from 2-4 independent experiments): (1) Axon segments 
for panel C: vehicle, $33 \mathrm{ctrl}$ and $32 \mathrm{KO}$; NAD': $17 \mathrm{ctrl}$ and $49 \mathrm{KO}$; NMN, 17ctrl and $45 \mathrm{KO}$; twoway ANOVA with Tukey's multiple comparisons. (2) Anterograde events for panel D: vehicle: 33 ctrl and $25 \mathrm{KO}$; NAD+, $17 \mathrm{ctrl}$ and $34 \mathrm{KO}$; NMN, $17 \mathrm{ctrl}$ and $31 \mathrm{KO}$. (3) Retrograde events for panel D: vehicle, $33 \mathrm{ctrl}$ and $29 \mathrm{KO}$; NAD+, $17 \mathrm{ctrl}$ and $34 \mathrm{KO}$; NMN, 17 ctrl and $31 \mathrm{KO}$; two-way ANOVA with Tukey's multiple comparisons.

Fig 6-S1. Supplementing with NAD+, NMN or Methyl-pyruvate rescues APP accumulation in NMNAT2 KO neurons

(A) Representative images of APP staining in DIV14 Ctrl and KO neuronal cultures supplemented with vehicle, NMN, NAD+ or Methyl-pyruvate (M-Pyruvate) from DIV8 till DIV14. (Scale bar $=30 \mu \mathrm{m})$. (B) Timeline of supplementation and sample collection. (C) Average total area of accumulated APP relative to $\mathrm{Ctrl}$ treated with vehicle, NMN, NAD+ and M-Pyruvate. (Vehicle: Ctrl N=54, KO=74; NMN: Ctrl N=30, KO N=45; NAD+: Ctrl N=48, KO N=77; Mpyruvate: $\mathrm{Ctrl} \mathrm{N}=23, \mathrm{KO} \mathrm{N}=32$. $\mathrm{N}$ represents image number from at least 2 batches of culture) Kruskal-Wallis test, ${ }^{* *} \mathrm{p}<0.01,{ }^{* * *} \mathrm{p}<0.0001$.

Fig 7. APP transport is dependent on glycolysis. Inhibiting glycolysis but not mitochondrial respiration prevents rescue of axonal transport by NAD+ or NMN supplementation in NMNAT2 KO neurons.

(A) Experimental timeline for drug treatment (grey bar), including vehicle, $1 \mu \mathrm{M}$ Oligomycin (Oligo), or $15 \mathrm{mM}$ 2-deoxyglucose with 10mM Methyl Pyruvate (2DG+Methyl-Pyr). (B) Schematic illustrating glycolysis and mitochondrial respiration affected by Oligo and 2DG+Methyl-Pyr treatments. The corresponding representative kymographs show APP transport in distal axons of DIV8 ctrl or KO neurons under the indicated treatments. Scale bar = $20 \mu \mathrm{m}$. (C) Average percentage of APP-EGFP vesicles exhibiting anterograde, retrograde and stationary movements in the distal axons of DIV8 ctrl and KO neurons in the presence of vehicle, Oligo and 2DG+Methyl-Pyr. (D,E) Summaries of APP-EGFP transport velocities. Oligo or 2DG treated groups were compared to the vehicle treated group in each data set. Axonal segments and statistics from two independent experiments: (1) Panel C: ctrl: 33 vehicle, 39 Oligomycin, and 20 2DG; KO+NAD+: 24 vehicle, 25 Oligomycin, 18 2DG; KO+NMN: 20 vehicle, 23 Oligomycin, 21 2DG; two-way ANOVA with Tukey's multiple comparisons. (2) Anterograde event numbers for panel D: ctrl: 45 vehicle, 39 Oligomycin, 10 2DG; KO+NAD+: 24 vehicle, 24 Oligomycin, 13 2DG; KO+NMN: 20 vehicle, 23 Oligomycin, 18 2DG; two-way ANOVA with Tukey's multiple comparisons. (3) Retrograde events for panel E: ctrl: 45 vehicle, 38 
Oligomycin, 10 2DG; two-way ANOVA with Tukey's multiple comparisons; KO+NAD+: 22 vehicle, 24 Oligomycin, 12 2DG; two-way ANOVA with Tukey's multiple comparisons; KO+NMN: 20 vehicle, 23 Oligomycin, 18 2DG; Kruskal-Wallis test.

Fig 8. Membrane-bound NMNAT2 reduces APP accumulation in NMNAT2 KO neurons.

(A) Experimental timeline. (B) Representative APP and HA double-staining images in DIV14 ctrl and KO neurons transduced with LV-copGFP, LV-WT-NMNAT2 and LV-PM-NMNAT2. Scale bar $=30 \mu \mathrm{m}$. (C) Summaries for normalized APP accumulation area. Number of images from 3 independent cultures used for analysis: LV-copGFP, 17 ctrl and $30 \mathrm{KO}$; LV-WT-NMNAT2, 24 ctrl and $34 \mathrm{KO}$; LV-PM-NMNAT2, 24 ctrl and $34 \mathrm{KO}$; two-way ANOVA with Tukey's multiple comparisons.

\section{Fig 8-S1. Comigration between APP and NMNAT2}

(A) Representative kymograph showing mCherry-tagged NMNAT2 comigrating with EGFPtagged APP in a DIV8 axon of Ctrl neuron. (Scale bar $=20 \mu \mathrm{m}$, total recording time $=30 \mathrm{~s}$ ). (B) Frequency distribution of anterograde and retrograde velocity of NMNAT2 and APP in DIV8 distal axons. (NMNAT2 $\mathrm{N}=445$, APP $\mathrm{N}=861, \mathrm{~N}$ represents the trajectory traced in all kymographs).

\section{Fig 8-S2. Comparison of lentiviral transduction rate}

(A) Diagram showing the composition of lentiviral vectors (B) Percentage of APP expressing neurons with detectable HA-tag expression at DIV14 since the time of addition of $3 \mathrm{MOI}$ or $8 \mathrm{MOI}$ lentivirus at DIV2. (3MOI: $\mathrm{Ctrl} \mathrm{N}=20, \mathrm{KO} \mathrm{N}=20$; $8 \mathrm{MOI}$ : $\mathrm{Ctrl} \mathrm{N}=12, \mathrm{KO} \mathrm{N}=18$. $\mathrm{N}$ represents image number.) Unpaired t test was used. (C) Western blot and quantification of WT-NMNAT2 and PM-NMNAT2 abundance normalized to actin loading control. 2MOI lentivirus was added at DIV2 and culture lysate was collected at DIV14. (LV-WT: Ctrl N=3, KO N=3; LV-PM: Ctrl N=3, $\mathrm{KO} N=3$. $\mathrm{N}$ represents biological repeats from independent wells.) One-way ANOVA, ${ }^{*} \mathrm{p}=$ 0.0251 . Data represents mean \pm SEM. 


\section{Material and Methods \\ Mice}

The generation and genotyping of NMNAT2-Blad mice have been described previously. In these mice, the NMNAT2 null mutation was generated by transposon-mediated gene-trap mutagenesis (41). The generation and genotyping of NMNAT2 ${ }^{\mathrm{f} / \mathrm{f}}$ mice have been described previously, in which a Cre recombinase dependent gene trap cassette was inserted within intron 1 of the NMNAT2 gene (79). The generation and genotyping of NEX-Cre mice have been described previously (32). NMNAT2/f/f mice (Fig. 1A) were crossed with NEX-Cre mice to knockdown NMNAT2 only in postmitotic cortical and hippocampal glutamatergic neurons from embryonic day 11.5. Mice with conditional NMNAT2 knockout show an ataxia phenotype with wobbling movements as well as a lighter body weight. $5 \mathrm{~g}$ DietGel $76 \mathrm{~A}\left(\mathrm{Clear}_{2} \mathrm{O}\right.$ ) was given daily after animals were weaned. Animal housing and use were in compliance with the NIH Guidelines for the Care and Use of Laboratory Animals and were approved by the institutional animal care and use committees at Indiana University and Max Planck Florida Institute for Neuroscience. NEX-Cre positive NMNAT2 ${ }^{\mathrm{f} /+}$ and NMNAT2 ${ }^{+/+}$and NEX-Cre negative NMNAT2/f/ littermates were used as control for NEX-Cre positive NMNAT2 ${ }^{\mathrm{f} / \mathrm{f}}$.

\section{Primary cortical neuronal cultures}

NMNAT2 KO cortical neurons and their corresponding WT or Het controls were dissected from the cortices of E15.5-16.5 embryos harvested from the NMNAT2-Blad heterozygous pregnant female mice crossed with NMNAT2-Blad heterozygous male mice. After dissection, the cortical tissue of each embryo was dissociated individually by using the Worthington Papain Dissociation Kit (Worthington Inc.) according to manufacturer's protocol. Genotyping was performed before the completion of papain dissociation. Cortices from WT and Het embryos were combined as control group for plating; the cortices from multiple $\mathrm{KO}$ embryos were combined as one experimental group for plating. Embryos of both sexes are included. For the RNA-seq study, neurons were dissected from E15.5-16.5 NMNAT2 ${ }^{\mathrm{f} / \mathrm{f}}$ embryos harvested from NMNAT2 ${ }^{\mathrm{f} / \mathrm{f}}$ female mice crossed with NMNAT2 $2^{\mathrm{f} / \mathrm{f}}$ male mice, following the same papain dissociation procedure without the need of genotyping. Embryos of both sexes are included.

Cortical neurons were maintained in Neurobasal Media (Gibco ${ }^{\mathrm{TM}}$, ThermoFisher Scientific) supplemented with 2\% B-27 (Gibco ${ }^{\mathrm{TM}}$ ), 2mM GlutaMAX ${ }^{\mathrm{TM}}$ (Gibco ${ }^{\mathrm{TM}}$ ) and $100 \mathrm{U} / \mathrm{ml}$ penicillinstreptomycin $\left(\mathrm{Gibco}^{\mathrm{TM}}\right)$. Neurons were incubated at $37^{\circ} \mathrm{C}$ with $5 \% \mathrm{CO} 2$ and appropriate humidity. One third of the media was replenished every 3 days. 


\section{Genotyping}

For mice, ear lysates were prepared by immersing the tissue derived from a small ear clip in digestion buffer $(50 \mathrm{mM} \mathrm{KCl}, 10 \mathrm{mM}$ Tris- $\mathrm{HCl}, 0.1 \%$ Triton $\mathrm{X}-100,0.1 \mathrm{mg} / \mathrm{ml}$ proteinase $\mathrm{K}, \mathrm{pH}$ 9.0), vortexing gently, and then incubating for $3 \mathrm{~h}$ at $60^{\circ} \mathrm{C}$ to lyse the cells. For embryos, the same digestion buffer with $0.2 \mathrm{mg} / \mathrm{ml}$ proteinase $\mathrm{K}$ was used, and embryonic tail lysates were incubated at $60^{\circ} \mathrm{C}$ for $15 \mathrm{~min}$ with $1500 \mathrm{rpm}$ shaking in a thermoshaker. These lysates were then heated to $95^{\circ} \mathrm{C}$ for 10 min to denature the proteinase $\mathrm{K}$ (Thermo Scientific, Rockford, IL, USA), and centrifuged at $16,100 \mathrm{~g}$ for $15 \mathrm{~min}$. The supernatants were used as DNA templates for polymerase chain reactions (PCRs, EconoTaq Plus Green 2X mater mix, Lucigen, Middleton, WI, USA). For embryonic genotyping during primary culture preparation, a QIAGEN Fast Cycling PCR Kit was used. The primers for genotyping are listed in key resource table.

\section{Immunohistochemistry and confocal imaging}

Mice were anesthetized and perfused with 4\% paraformaldehyde (PFA). Brains were harvested and post-fixed with $4 \%$ PFA overnight at $4^{\circ} \mathrm{C}$. Fixed brains were sectioned into $40-\mu \mathrm{m}$-thick sections in the coronal plane by using a vibrating microtome (VT-1000 Vibrating Microtome, or SM-2000R, Leica Microsystems). Free-floating sections were used in all subsequent steps. Sections were permeabilized with $0.3 \%$ Triton X-100 in PBS for $20 \mathrm{~min}$ at room temperature, incubated with blocking solution (3\% normal goat serum prepared in PBS with $0.3 \%$ Triton X100) for $1 \mathrm{~h}$, and then incubated with primary antibodies diluted in blocking solution overnight at $4^{\circ} \mathrm{C}$. The next day, sections were washed with $0.3 \%$ Triton PBS for 3 times and then incubated with the secondary antibodies diluted in blocking solution at $4^{\circ} \mathrm{C}$ for $2 \mathrm{~h}$. After the incubation, samples were washed with $0.3 \%$ Triton PBS for 3 times. Draq5 (1:10,000 dilution, Cell Signaling) or 4',6-diamidino-2-phenylindole (DAPI, $5 \mu \mathrm{g} / \mathrm{ml}$, Invitrogen) were added during the first wash step to visualize nuclei. Dako Mounting Medium was used to mount the brain sections. Single plane fluorescent images were taken by a Leica TCS SPE confocal microscope with a $10 x$ objective lens (0.3 N.A.). A minimum of three sections were imaged per mouse, and each anatomical region imaged was comparable across all animals. DAPI/Draq5 immunofluorescence was used to match corresponding sections and find the same anatomical landmarks to image. The total pixel value in the fluorescent channel corresponding to APP was measured by Image J.

\section{Immunocytochemistry and confocal imaging}


About $1.3^{\star} 10^{3}$ cells $/ \mathrm{mm}^{2}$ cortical neurons were plated on poly-D-lysine (PDL)-coated $12 \mathrm{~mm}$ diameter coverslips in a 24 well plate (about $2.5^{\star} 10^{5}$ cells onto $190 \mathrm{~mm}^{2}$ surface). At DIV14, cortical neurons were fixed by $4 \%$ PFA in $4 \%$ sucrose for $20 \mathrm{~min}$, then permeabilized and blocked in PBS containing 0.1\% Triton X-100 and 5\% Goat Serum for 60 min, and then incubated with primary antibody diluted in $5 \%$ Goat Serum $+0.1 \%$ Triton PBS overnight at $4{ }^{\circ} \mathrm{C}$ with rotation. Then, samples were washed with $0.1 \%$ Triton PBS 3 times and incubated with secondary antibody diluted in $5 \%$ Goat Serum $+0.1 \%$ Triton PBS for 2 hours with rotation. After the incubation, samples were washed with $0.1 \%$ Triton PBS 3 times. ProLong ${ }^{\mathrm{TM}}$ Gold Antifade Mountant with DAPI was used to mount the coverslips.

For APP staining, images were taken by a Leica TCS SPE confocal microscope using a 1.4 N.A. $63 x$ oil objective lens with $0.5 \mu \mathrm{m}$ z-step size to cover the whole depth of the cultured neurons. Five regions were randomly selected from each coverslip to image accumulated APP. Each image accounts for one data point to compare the degree of APP accumulation. Two coverslips per group were imaged for each culture. For MAP2 and $\beta$ III-tubulin staining, confocal images were taken by a Nikon A1R-HD25 laser scanning confocal microscope (Nikon Instruments) using a 1.4 N.A. Apo Lambda S $60 x$ oil objective lens with $0.5 \mu \mathrm{m} z$-step size to cover the whole depth of the neuron. "Large image stitching" with 5-10\% overlap was used to tile 4 adjacent fields of view together as one image to sample a larger area. Five regions were randomly selected from each coverslip and two coverslips per group were imaged for each culture.

\section{APP accumulation and gross neurite growth quantification}

ImageJ was used for quantification of the maximum intensity projected images. APP signal in somata and dendrites was manually excluded, then the default threshold method was applied to select the top $10 \%$ tail of total pixel, then the "analyze particles" function was applied to select particles of area larger than $0.9 \mu \mathrm{m}^{2}$ which were identified as "accumulated APP". The total area of "accumulated APP" was calculated in each image to evaluate the phenotype. MAP2 positive area was identified by means of an auto-thresholding method and measured for each image. MAP2 positive area was selected by "create selection" function after thresholding, then restored and deleted from the $\beta$ III-tubulin channel. Therefore, the remaining $\beta$ III-tubulin signal solely represented axonal regions. Similarly, mean threshold was applied to measure the $\beta$ III-tubulin positive area. Live cells were distinguished by DAPI-stained nuclei morphology, and the number of live cells was determined for each image. MAP2 and $\beta$ III-tubulin area were normalized by 
dividing by the live cell number in each image. We observed a skew in the data if the cell density was dramatically different between Ctrl and KO. Therefore, only images with a live cell number between 150 to 300 within $0.31 \mathrm{~mm}^{2}$ image area (485-970 cells $\left./ \mathrm{mm}^{2}\right)$ were used for the analysis.

\section{Plasmid DNA constructs}

pEGFP-n1-APP was a gift from Zita Balklava \& Thomas Wassmer (Addgene plasmid \# 69924; http://n2t.net/addgene:69924; RRID: Addgene_69924) (80). pEGFP-c1-SNAP25 and pmCherryN1-NMNAT2 were gifts from Michael Coleman \& Jonathan Gilley (27). pCX-EGFP was a gift from Matthew Neil Rasband (81). pLV-mitoDsRed was a gift from Pantelis Tsoulfas (Addgene plasmid \# 44386; http://n2t.net/addgene:44386; RRID: Addgene_44386) (82). pcDNA3.1-SoNar and pcDNA3.1-cpYFP were gifts from Yang Yi (52). pCMV-MitoVenus was a gift from Lulu Cambronne (26). pCAG-mCherry was a gift from Ken Mackie. pcDNA3-Syn-ATP and pcDNA3Cyto-pHluorin were from Vidhya Rangaraju (53).

\section{Neuronal transfection}

For lipofectamine transfection, about $1.66^{*} 10^{3}$ cells $/ \mathrm{mm}^{2}$ cortical neurons were plated on PDL (Poly-D-Lysine)-coated MatTek dish (P35G-1.5-20-C) (about $1.6^{\star} 10^{6}$ cells on the whole surface of the $35 \mathrm{~mm}$ diameter dish). For APP-EGFP and SNAP25-EGFP transport imaging, cortical neurons were transfected $16 \mathrm{hr}$ to $20 \mathrm{hr}$ prior to the time-lapse imaging experiments using Lipofectamine 3000 (Thermo) following manufacturer's instruction. For MitoDsRed transport imaging, cortical neurons were cotransfected with pCX-EGFP and pLV-mitoDsRed 36-48 hr before live imaging using Lipofectamine 3000. Before adding the DNA-lipofectamine mixture, half of the conditioned culture medium was removed and saved for later. Two hours after incubation with DNA-lipofectamine mixture, one third of the remaining medium was further removed and $2 \mathrm{ml}$ of culture medium (1:1 of conditional medium and fresh medium) was added back immediately. Right before imaging, all culture medium was replaced by 2-3 $\mathrm{ml}$ of Hibernate E low fluorescence buffer (BrainBits) supplemented with $2 \mathrm{mM}$ GlutaMAX ${ }^{\mathrm{TM}}$ to maintain the ambient pH environment.

For immunocytochemistry, about $1.3^{\star} 10^{3}$ cells $/ \mathrm{mm}^{2}$ cortical neurons were plated on PDL-coated $12 \mathrm{~mm}$ diameter coverslips in a 24-well-plate (about $2.5^{\star} 10^{5}$ cells on $190 \mathrm{~mm}^{2}$ surface) and 3MOI (multiplicity of infection) or 8MOI lentivirus was applied at DIV2. For RNA extraction or Western 
blotting, $1.3^{*} 10^{3}$ cells $/ \mathrm{mm}^{2}$ cortical neurons were plated on PDL-coated 12-well-plate (about $5^{\star} 10^{5}$ cells on $380 \mathrm{~mm}^{2}$ surface) and $2 \mathrm{MOI}$ lentivirus was applied.

For magnetofection, about $1.66^{\star} 10^{3}$ cells $/ \mathrm{mm}^{2}$ cortical neurons were plated on the center of a PDL-coated coverglass region of MatTek dish (P35G-1.5-14-C) (about $2.56^{\star} 10^{5}$ cells onto $153.86 \mathrm{~mm}^{2}$ surface). For Syn-ATP imaging and Cyto-pHluorin imaging, cortical neurons were transfected $24 \mathrm{hr}$ prior to imaging using Combimag (OZ biosciences) and Lipofectamine 2000 (Invitrogen) according to manufacturer's instructions. Briefly, $0.5 \mu \mathrm{g}$ DNA was incubated with 0.5 $\mu \mathrm{l}$ lipofectamine in $50 \mu$ l transfection medium (Neurobasal media supplemented with $2 \mathrm{mM}$ Glutamax without B27 or antibiotics) for $5 \mathrm{~min}$, then mixed with $0.5 \mu$ l Combimag diluted in another $50 \mu$ transfection medium for $10 \mathrm{~min}$. The DNA-lipofectamine-Combimag mixture was further diluted in $125 \mu \mathrm{l}$ of transfection medium. Then, the conditioned medium from cultured neurons was harvested and the neurons were immediately rinsed twice in warm transfection medium. Transfection medium used for rinsing was removed and $150 \mu$ of DNA-lipofectamineCombimag mixture was added to the neurons. Neurons were placed on a magnetic plate for 20 min inside a $37^{\circ} \mathrm{C}$ and $5 \% \mathrm{CO}_{2}$ incubator. After $20 \mathrm{~min}$, neurons were rinsed once in warm transfection medium and replaced with the previously harvested warm, conditioned medium.

\section{NAD ${ }^{+}$and NMN supplementation}

$\mathrm{NAD}^{+}$(Roche, NAD100-RO) or NMN (Sigma, N3501) was dissolved in 1xPBS at a stock concentration of $100 \mathrm{mM}$, aliquoted and stored at $-80^{\circ} \mathrm{C}$. Only less than 1 -week-old stocks were used. At DIV5, NAD+ or NMN was applied to the culture medium at a final concentration of 1 $\mathrm{mM}$. At DIV6 and DIV7, 1/3 of culture medium was replaced with fresh medium containing $1 \mathrm{mM}$ $\mathrm{NAD}^{+}$or NMN. If the transfection was conducted at DIV7, conditioned medium containing $1 \mathrm{mM}$ $\mathrm{NAD}^{+}$or NMN was added back after transfection. At DIV8, 1 mM NAD ${ }^{+}$or NMN was supplemented to the Hibernate $\mathrm{E}$ low fluorescence buffer used for live imaging or DMEM during seahorse assay. Unless specified otherwise, all live imaging experiments were done in Hibernate E low fluorescence buffer.

\section{Axonal transport timelapse video microscopy and movie analyses}

Live imaging of neuronal cultures was carried out using an Olympus OSR Spinning Disk Confocal microscope (CSU-W1, Yokogawa) connected to a Hammamatsu Flash 4 V2 camera. Temperature was maintained at $37^{\circ} \mathrm{C}$ by a Tokai Hit Stage Top Incubation system. For APPEGFP and SNAP25-EGFP transport recording, movies were taken in axonal segments at least 
$400 \mu \mathrm{m}$ away from the soma (defined as distal axon) and axonal segments within $200 \mu \mathrm{m}$ of the soma (defined as proximal axon) and captured at a rate of 5 frames/s for a total recording time of $60 \mathrm{~s}$ with a $63 \mathrm{x}$ oil objective (1.46 N.A.). For MitoDsRed transport imaging, neurons were cotransfected with EGFP, and the distance from axonal segment of interest to soma was determined in the GFP channel under the eyepiece. Mitochondria transport videos were taken in the distal region at a 3 second time interval for a total recording time of 15 min with a $63 \mathrm{X}$ oil objective (1.46 N.A.). A z-stack with 1 to $1.5 \mu \mathrm{m}$ z-step size was taken for each time point to cover all the mitochondria within the imaged axonal segment.

Kymographs were generated using ImageJ (http://rsb.info.nih.gov/ij) with "Velocity_Measurement_Tool” macro (http://dev.mri.cnrs.fr/projects/imagejmacros/wiki/Velocity_Measurement_Tool). Quantification of velocity was manually performed by following the trajectory of each particle with an angle larger than $0^{\circ}$ relative to the time axis. Stationary and repetitive bidirection-moving particles were excluded for velocity measurements. If an anterograde or retrograde moving particle stopped for a short while during the imaging and then continued moving, the paused portion was included in the velocity calculation. Although a portion of the axon could be out of focus, resulting in a discontinuous trajectory, the discontinuous trajectory was still traced as the same if the slope and timing matched. All the traced trajectories were saved in "ROI manager" in ImageJ. Particles that remained stationary or underwent repetitive bidirectional movements for the majority of the recording time were defined as stationary/dynamic pause events. Each trajectory of stational/dynamic pause particles that could be visually separated was included in the number of stationary/dynamic pause events. The number of anterograde or retrograde events was counted in the same way. Stationary/dynamic pause percentage was calculated as the number of stationary/dynamic events divided by the sum of the number of anterograde, retrograde, and stationary/dynamics pause events. Similarly, anterograde and retrograde percentages were calculated.

\section{2-Deoxyglucose (2DG) and oligomycin treatments}

To acutely inhibit glycolysis while leaving mitochondrial respiration intact, 15 mM 2deoxyglucose and $10 \mathrm{mM}$ methyl-pyruvate were added to the customized Hibernate $\mathrm{E}$ low fluorescence buffer containing $0 \mathrm{mM}$ glucose ( $262 \mathrm{mmol} / \mathrm{kg}$ osmolality). Culture medium was replaced by the 2DG Hibernate $E$ buffer and neurons were incubated for 30 min before imaging APP-EGFP axonal transport. For each culture dish, imaging of axonal transport was conducted within 30 min of 2DG treatment. To acutely inhibit ATP synthesized from mitochondrial 
respiration, $1 \mu \mathrm{M}$ oligomycin was added to the commercial Hibernate $\mathrm{E}$ low fluorescence buffer containing $25 \mathrm{mM}$ glucose ( 241 mmol/kg osmolality). Culture medium was replaced by Oligo Hibernate $E$ buffer and neurons were incubated for 5 min before imaging APP-EGFP axonal transport, Syn-ATP or Cyto-pHluorin. For each culture dish, live imaging was conducted within 30 min of oligomycin treatment. For APP axonal transport imaging, oligomycin (Calbiochem 495455) was dissolved in DMSO to prepare a $1 \mathrm{mM}$ stock. For Syn-ATP and Cyto-pHluorin imaging, oligomycin (Sigma-Aldrich O4876) was dissolved in 100\% Ethanol to prepare a $1 \mathrm{mM}$ stock.

\section{NAD+/NADH-Glo ${ }^{\text {TM }}$ bioluminescent assay}

Control or KO neurons were cultured in a 96-well plate at a density of $1.3^{\star} 10^{3}$ cells $/ \mathrm{mm}^{2}$ (4.235* $10^{4}$ cells on $32 \mathrm{~mm}^{2}$ surface). Following manufacturer's instruction (Promega), neuronal cultures at DIV8 were washed three times with PBS and lysed in buffer containing $25 \mu$ PBS and $25 \mu \mathrm{l} 1 \%$ DTAB (Dodecyltrimethylammonium bromide) base buffer (100 mM Sodium Carbonate, 20 mM Sodium Bicarbonate, 10 mM Nicotinamide, $0.05 \%$ TritonX-100, pH 10-11). $30 \mu \mathrm{l}$ of lysate was collected for BCA protein assay. $20 \mu \mathrm{l}$ of the remaining lysate was diluted into $105 \mu \mathrm{l} .50 \mu \mathrm{l}$ of diluted lysate was mixed with $25 \mu \mathrm{l} 0.4 \mathrm{~N} \mathrm{HCl}$ and incubated at $60{ }^{\circ} \mathrm{C}$ for 15 min to detect $\mathrm{NAD}^{+}$; while another $50 \mu \mathrm{l}$ of diluted lysate (without $0.4 \mathrm{~N} \mathrm{HCl}$ ) was incubated at $60^{\circ} \mathrm{C}$ for $15 \mathrm{~min}$ to detect $\mathrm{NADH}$. After incubation, $25 \mu \mathrm{l}$ of $0.5 \mathrm{M}$ Tris base was added to the wells used for detecting $\mathrm{NAD}^{+}$to neutralize the $\mathrm{HCl} ; 50 \mu$ of a solution containing $1: 10.4 \mathrm{~N} \mathrm{HCl}$ and $0.5 \mathrm{M}$ Tris base was added to wells used for detecting $\mathrm{NADH}$. Freshly dissolved $\mathrm{NAD}^{+}$in PBS was used to prepare the standard curve. Luminescent signal from each sample was converted into absolute $\mathrm{NAD}^{+}$or $\mathrm{NADH}$ concentration and normalized to protein concentration measured by the BCA method (Thermo Scientific ${ }^{\mathrm{TM}}$ ).

\section{NAD+NADH live imaging using the genetically encoded sensor, SoNar}

$\sim 1.66^{\star} 10^{3}$ cells $/ \mathrm{mm}^{2}$ cortical neurons were plated on PDL (Poly-D-Lysine)-coated MaTek dish (P35G-1.5-20-C). The NAD+/NADH sensor, SoNar, and its control cpYFP were gifts from Yi Yang's group. On DIV6, 2.5 ug DNA of pCDNA3.1-SoNar or pCDNA3.1-cpYFP were transfected. After $48 \mathrm{hr}$ expression of the sensor or control (DIV8), the culture medium was replaced by Hibernate $\mathrm{E}$ buffer and maintained at $37^{\circ} \mathrm{C}$ for imaging using a Leica TCS SP8 confocal laser scanning platform with HyD hybrid detector and a HC PL APO 40x (1.2 NA) water-immersion objective. The SoNar sensor/cpYFP was excited at $440 \mathrm{~nm}$ and $488 \mathrm{~nm}$ and the emission at $530+/-15 \mathrm{~nm}$ was detected to obtain the ratiometric measurement. Laser power 
intensity was maintained the same between WT/Het and $\mathrm{KO}$ in the same experiment. Axonal segment $>400 \mu \mathrm{m}$ away from the soma (defined as distal axon) and axonal segment within 200 $\mu \mathrm{m}$ to the soma (defined as proximal axon) were imaged with $1 \mu \mathrm{m} z$-step size to cover the whole axon segment within the field of view. Maximum intensity projection was generated for further analysis. Mean grey value from $488 \mathrm{~nm}$ excitation (F488) and $440 \mathrm{~nm}$ excitation (F440) was measured in ImageJ using manually drawn regions of interest (ROIs) that circle the edges of the axons of interest. Five rectangular ROls were randomly drawn in the background area and the average mean grey value from these five ROls was used to subtract background. After background subtraction, the ratio was calculated as (F488 axon $\left.-\mathrm{F} 488_{\text {background }}\right) /\left(\mathrm{F}_{440}\right.$ axon $^{-}$ F440 background). Data presented were normalized to control as percentage.

\section{Glycolytic rate assay}

Glycolytic rates of control and KO primary cortical neurons was measured in an XFe24 Extracellular Flux Analyzer (Seahorse Bioscience) maintained at $37^{\circ} \mathrm{C}$. Both control and $\mathrm{KO}$ neurons were plated at a density of $3.63^{*} 10^{3}$ cells $/ \mathrm{mm}^{2}$ in XF24 cell-culture microplates (Seahorse Bioscience) (about $10^{*} 10^{4}$ cells on $27.5 \mathrm{~mm}^{2}$ surface). This seeding density gave the optimal basal ECAR range between $20-120(\mathrm{mpH} / \mathrm{min})$ in control cortical neurons at DIV8, as recommended by the manufacturer. On DIV8, neuronal cultures were washed once with warm $1 \mathrm{ml}$ XF DMEM ( $\mathrm{pH} 7.4$, supplemented with $10 \mathrm{mM}$ glucose, $1 \mathrm{mM}$ pyruvate and $2 \mathrm{mM}$ glutamine) and then incubated in $1 \mathrm{ml} \mathrm{XF} \mathrm{DMEM} \mathrm{medium} \mathrm{at} 37^{\circ} \mathrm{C}$ in a non- $\mathrm{CO}_{2}$ incubator for $60 \mathrm{~min}$ prior to the assay. Before starting the assay, $1 \mathrm{ml} \mathrm{XF} \mathrm{DMEM} \mathrm{was} \mathrm{replaced} \mathrm{with} \mathrm{fresh}$ and warm XF DMEM in $500 \mu \mathrm{l}$ total volume. The extracellular acidification rate (ECAR) and oxygen consumption rate (OCR) were measured as baseline for $24 \mathrm{~min}$ (3 cycles, each cycle spends 3 min for mixing, 2 min for waiting and 3 min for measurement); then 15 uM NMDA (Sigma) and 2 uM Glycine (Sigma) were injected and measured for another $24 \mathrm{~min}$; then $0.5 \mu \mathrm{M}$ rotenone and antimycin were injected and measured for another $24 \mathrm{~min}$; then $50 \mathrm{mM} 2$ deoxyglucose was injected and measured for another $40 \mathrm{~min}$ (5 cycles). At the end of the assay, XF DMEM was removed, and neurons were lysed in $30 \mu \mathrm{l}$ Tris-based lysis buffer (150 $\mathrm{mM} \mathrm{NaCl}, 50 \mathrm{mM}$ Tris $\mathrm{HCl}, \mathrm{pH}$ 7.6, $0.1 \%$ Triton and protease inhibitor) and total protein quantity was measured by Bradford assay (Bio-Rad) for normalization. The assay buffer factor for the default protocol is $2.5(\mathrm{mmol} / \mathrm{L} / \mathrm{pH})$ and was used to calculate proton efflux rate (PER). PER contributed from mitochondrial respiration was estimated by OCR and deducted to represent PER solely from glycolysis (glycoPER). Unless otherwise specified, reagents were from the glycolytic rate assay kit (Seahorse Bioscience). 


\section{Mito stress assay}

Oxygen consumption rate (OCR) in control and KO primary cortical neurons was measured in an XFe24 Extracellular Flux Analyzer (Seahorse Bioscience) maintained at $37^{\circ} \mathrm{C}$. Both control and $\mathrm{KO}$ neurons were plated at a density of $1.45^{\star} 10^{3}$ cells $/ \mathrm{mm}^{2}$ in XF24 cell-culture microplates (Seahorse Bioscience) (about $4{ }^{*} 10^{4}$ cells on $27.5 \mathrm{~mm}^{2}$ surface). This seeding density gave the optimal basal OCR range between 50-400 (pmol/min) in control cortical neurons at DIV8, as recommended by the manufacturer. On DIV8, neuronal culture medium was replaced by $1 \mathrm{ml}$ XF DMEM medium ( $\mathrm{pH} 7.4$, supplemented with $10 \mathrm{mM}$ glucose, $1 \mathrm{mM}$ pyruvate and $2 \mathrm{mM}$ glutamine) and incubated at $37^{\circ} \mathrm{C}$ in a non- $\mathrm{CO}_{2}$ incubator for $60 \mathrm{~min}$ prior to the assay. Before starting the assay, $1 \mathrm{ml}$ XF DMEM was replaced with fresh and warm XF DMEM in $500 \mu$ lotal volume. Oxygen consumption rate (OCR) was measured as baseline for 16 min (2 cycles, each cycle spends 3 min for mixing, 2 min for waiting and 3 min for measurement); then $15 \mathrm{uM}$ NMDA (Sigma) and 2 uM Glycine (Sigma) were injected and measured for another 16 min; then $1 \mathrm{uM}$ oligomycin was injected and measured for another $16 \mathrm{~min}$; then 2 uM FCCP was injected and measured for another $24 \mathrm{~min}$ ( 3 cycles); then $0.5 \mu \mathrm{M}$ rotenone and antimycin were injected and measured for another $24 \mathrm{~min}$. At the end of the assay, XF DMEM was removed, and cells were lysed in $30 \mu \mathrm{l}$ Tris-based lysis buffer $(150 \mathrm{mM} \mathrm{NaCl}, 50 \mathrm{mM}$ Tris $\mathrm{HCl}, \mathrm{pH}$ 7.6, $0.1 \%$ Triton and protease inhibitor) and total protein quantity was measured by Bradford assay (Bio-Rad) for normalization. Unless otherwise specified, reagents were from Mito stress assay kit (Seahorse Bioscience).

\section{Mitochondria density and morphology analysis}

Both control and $\mathrm{KO}$ neurons were plated at a density of $1.8^{*} 10^{3}$ cells $/ \mathrm{mm}^{2}$ on PDL-coated $12 \mathrm{~mm}$ diameter coverslip in 24-well culture plate (about $3.5^{\star} 10^{5}$ cells on $190 \mathrm{~mm}^{2}$ surface). Neurons were co-transfected with pCMV-MitoVenus and pCAG-mCherry at DIV6 and fixed using 4 \%PFA with $4 \%$ Sucrose at DIV8. Immunocytochemistry with antibodies against GFP (Chicken) and RFP (Rabbit) was conducted to immunoamplify the MitoVenus and mCherry signals after fixation. ProLong ${ }^{\text {TM }}$ Gold Antifade Mountant with DAPI was used to mount the coverslips.

Images were taken by a Nikon A1 laser scanning confocal microscope using a 1.4 N.A. Apo Lambda S 60x oil objective lens with $0.171 \mu \mathrm{m}$ z-step size and 3 times zoom to cover the whole depth of the axon segment of interest. Axonal segments at least $400 \mu \mathrm{m}$ away from the soma 
were identified in the RFP channel and selected for imaging. Distal axons from at least 8-10 neurons were randomly sampled per coverslip and two coverslips were imaged per group. Images were analyzed using Imaris. RFP channel was used to generate the surface masking of the axonal region, within which the MitoVenus signal was used to create the surface representing mitochondrial morphology. To calculate mitochondria density, length of the axon segment in each image was measured. Density was calculated from mitochondria number divided by axonal length in each image. Sphericity values of mitochondrial surface from axon segments of each individual neuron were inputted in each column in the column table in Prism 9.0 (each column representing one individual neuron). Cumulative frequency distribution of relative percentage was generated through "column analysis" function in Prism 9.0. Statistical analysis of cumulative frequency distribution is described in "quantification and statistical analysis" section.

\section{Western blotting}

Control or KO cortical neurons were plated at a density around $1.3^{*} 10^{3}$ cells $/ \mathrm{mm}^{2}$ on PDL coated-12-well-plate (about $5^{\star} 10^{5}$ cells on $380 \mathrm{~mm}^{2}$ surface). $2 \mathrm{MOI}$ lentivirus expressing WTNMNAT2 or PM-NMNAT2 was applied at DIV2. At DIV14, cultured cells were lysed in 1\% TritonX-100 lysis buffer [20 mM of Tris-base ( $\mathrm{pH} 7.4$ ), $137 \mathrm{mM}$ of $\mathrm{NaCl}, 1 \%$ Triton X-100, 10\% glycerol, $2 \mathrm{mM}$ of EDTA, protease inhibitor cocktail and phosphatase inhibitor cocktail]. The supernatant solution was collected using centrifugation at $10000 \times \mathrm{g}$ for 5 min at $4{ }^{\circ} \mathrm{C}$. Protein concentration was measured using the BCA method (Thermo Scientific ${ }^{\mathrm{TM}}$ ). Proteins were separated on a $10 \%$ SDS-polyacrylamide gel and then transferred to a nitrocellulose membrane (BioRad). The membrane was incubated with the appropriate primary antibody and then incubated with a species-appropriate secondary antibody. Western blot images were acquired by LI-COR Odyssey scanner and software (LI-COR Biosciences, Lincoln, NE USA) and quantified with NIH ImageJ software.

\section{Syn-ATP live imaging}

Syn-ATP imaging was performed using a custom-built inverted spinning disk confocal microscope (3i imaging systems; model CSU-W1) with two cameras: an Andor iXon Life 888 for confocal fluorescence imaging and an Andor iXon Ultra 888 electron-multiplying charge-coupled device camera for luminescence imaging. The Andor iXon Ultra 888 camera was selected for ultralow dark noise that was further reduced by cooling to $-100^{\circ} \mathrm{C}$. The speed of the Andor iXon Ultra 888 camera used for luminescence measurements was $1 \mathrm{MHz}$ with 1.00 gain and 1000 
intensification. Image acquisition was controlled by Slidebook 6 software. Confocal imaging of mCherry fluorescence was performed by illuminating neurons with a $561 \mathrm{~nm}$ laser with $200 \mathrm{~ms}$ exposure and $2.3 \mathrm{~mW}$ laser power. For mCherry fluorescence measurements, ten frames of timelapse imaging without interval were acquired and the average of ten images was used to measure the mCherry fluorescence signal from the varicosities of interest. For luminescence measurements, luminescence photons were collected by accumulating the image for $60 \mathrm{~s}$ in the presence of $2 \mathrm{mM}$ D-luciferin (Promega), and the luminescence signal was measured from the same varicosities as the corresponding fluorescence image. All images were acquired through a Plan-Apochromat 63x/1.4 N.A. Oil objective, M27 with DIC III prism, using a CSU-W1 Dichroic for $561 \mathrm{~nm}$ excitation with Quad emitter and individual emitters for confocal fluorescence, and a $720 \mathrm{~nm}$ multiphoton short-pass emission filter was used for luminescence. During imaging, temperature was maintained at $37^{\circ} \mathrm{C}$ by means of an Okolab stage top incubator with temperature control. Distal axon at least $400 \mu \mathrm{m}$ away from the soma was selected for imaging.

Images were analyzed in ImageJ using the plugin, Time series analyzer (https://imagej.nih.gov/ij/plugins/time-series.html). Regions of Interest (ROls) of $\sim 1.2 \mu \mathrm{m}$ diameter were drawn around the varicosities of interest to obtain the mean fluorescence and luminescence values from the corresponding fluorescence and luminescence images. We observed that some presynaptic varicosities in DIV8 neurons are not mature and stable over the course of imaging, and have a heterogenous amount of synaptophysin expression, consistent with previous studies (83). Therefore, we focused on varicosities of at least $1.2 \mu \mathrm{m}$ diameter and that were stable during the 60s luminescence acquisition. Neurons with an average mCherry fluorescence value lower than 1000 A.U. in their varicosities were excluded from the analysis, as low fluorescence value indicates low expression of the sensor and therefore resulted in low luminescence signal-to-noise ratio. For background subtraction of the fluorescence and luminescence values, three rectangular ROls were drawn in the background region surrounding axons of interest. The average mean values from these background regions in the fluorescence ( $\left.F_{\text {background }}\right)$ and luminescence ( $\left.L_{\text {background }}\right)$ channels were used to subtract background for each individual varicosity from the fluorescence $\left(F_{\text {varicosity }}\right)$ and luminescence ( $\left.L_{\text {varicosity }}\right)$ channels, respectively.

$$
\frac{L}{F}=\left(L_{\text {varicosity }}-L_{\text {background }}\right) /\left(F_{\text {varicosity }}-F_{\text {background }}\right)
$$

The average L/F value across varicosities within one neuron was used to represent the relative sv-ATP level for that neuron, accounting for one data point in the statistical analysis. 


\section{pH measurement and pH correction of L/F measured from Syn-ATP}

Cytoplasmic pHluorin (Cyto-pHluorin) was transfected in cortical neurons at DIV7 and imaging was done at DIV8 using the same equipment setting as Syn-ATP measurements. Detail procedures are described in the previous publication (53). In brief, neuronal culture medium was replaced by Tyrodes buffer containing (in mM) $119 \mathrm{NaCl}, 2.5 \mathrm{KCl}, 2 \mathrm{CaCl}_{2}, 2 \mathrm{MgCl}_{2}, 30 \mathrm{HEPES}$ (buffered to $\mathrm{pH} 7.4$ at $37^{\circ} \mathrm{C}$ ) and 25 glucose. Basal fluorescence intensity of Cyto-pHluorin was monitored for $5 \mathrm{~min}$, acquired every $15 \mathrm{~s}$ (20 timepoints). ROls were drawn around the axonal segment of interest and the mean fluorescence value was measured over the 20 timepoints $\left(F_{\text {axon, frame }}\right)$. Three rectangular ROls were drawn in the background region surrounding the axon

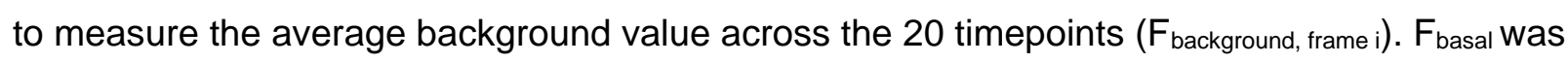
calculated by subtracting background in each timepoint and then averaging the value across the 20 timepoints.

$$
F_{\text {basal }}=\frac{\sum_{20 \text { frames }}\left(F_{\text {axon, frame } i}-F_{\text {background, frame } i}\right)}{20}
$$

Then, $1 \mu \mathrm{M}$ Oligomycin was added to the Tyrodes buffer and incubated for $5 \mathrm{~min}$. Fluorescence of Cyto-pHluorin was imaged to obtain Foligo using the above calculation.

Then, Tyrodes buffer was replaced by an $\mathrm{NH}_{4} \mathrm{Cl}$ solution containing (in $\mathrm{mM}$ ) $50 \mathrm{NH}_{4} \mathrm{Cl}, 70 \mathrm{NaCl}$, $2.5 \mathrm{KCl}, 2 \mathrm{CaCl}_{2}, 2 \mathrm{MgCl}_{2}, 30$ HEPES (buffered to $\mathrm{pH} 7.4$ at $37^{\circ} \mathrm{C}$ ) and 25 glucose. The response of Cyto-pHluorin to $\mathrm{NH}_{4} \mathrm{Cl}$ treatment was monitored for $5 \mathrm{~min}$, every $15 \mathrm{~s}$ (20 timepoints). The measured fluorescence of Cyto-pHluorin was called $F_{M a x}$, and was background subtracted as explained above.

Cytosolic $\mathrm{pH}$ of the basal and Oligomycin treatments were determined using the following modified Henderson-Hasselbalch equation:

$$
\begin{aligned}
& p H_{\text {Basal }}=\mathrm{pKa}-\log \left[\left(\frac{1+10^{\text {pKa-7.4 }}}{\frac{F_{\text {Basal }}}{F_{\text {Max }}}}\right)-1\right] \quad p H_{\text {Oligo }}=\mathrm{pKa}-\log \left[\left(\frac{1+10^{\text {pKa-7.4 }}}{\frac{F_{\text {Oligo }}}{F_{\text {Max }}}}\right)-1\right] \\
& p K a=7.1 \text { for CytopHluorin }
\end{aligned}
$$

From the known $\mathrm{pH}$ values at various conditions, Syn-ATP L/F value was corrected as follows:

$$
\begin{gathered}
L_{1} \sim \text { Luminescence under } p H_{1} \quad L_{0} \sim \text { Luminescence under } p H_{0} \\
\qquad p H_{1}=\mathrm{pKa}-\log \left[\left(\frac{1+10^{p K a-p H_{0}}}{\frac{L_{1}}{L_{0}}}\right)-1\right]
\end{gathered}
$$




$$
L_{0}=L_{1} *\left(\frac{1+10^{p K a-p H_{1}}}{1+10^{p K a-p H_{0}}}\right) \quad p K a=7.03 \text { for SynATP }
$$

Here, the average $\mathrm{pH}$ in each condition was used for correction and we did not propagate errors of $\mathrm{pH}$ measurements into the final error shown in L/F measurements. $\mathrm{pH}$ corrections of L/F values were only done for those conditions that showed a statistically significant change in $\mathrm{pH}$ compared to control (as in Fig 8-S1).

\section{Experimental Design}

No statistical methods were used to predetermine the sample size. Experiments were not randomized. During experiments, investigators were not blinded to the allocation of control versus experimental groups and treatments. During quantification of APP accumulation and axonal transport, investigators were blinded to the genotype and treatment information of each image. During quantification of $\mathrm{NAD}^{+} / \mathrm{NADH}$ sensor imaging and Syn-ATP sensor imaging, the investigator was not blinded to the genotype of each image. For NAD/NADH-Glo ${ }^{\mathrm{TM}}$ bioluminescent assay and Seahorse assays, no human assessment was involved for quantification. Inclusion and exclusion criteria were used in the analysis of Seahorse assays (glycolytic rate assay and mito stress assay) to avoid wells with technical issues featured by abnormally high oxygen level, as suggested by Agilent technical support. Inclusion and exclusion criteria were used in the analysis of Syn-ATP sensor imaging to avoid varicosities with extremely low mCherry (control construct) expression. Except gross neurite growth analysis at DIV5 \& DIV14 and Cyto-pHluorin imaging, all experiments were replicated with neurons from at least 2 independent primary culture preparations, as detailed in the figure legends.

\section{Quantification and statistical analysis}

Images were analyzed using Fiji (Image $\mathrm{J}$ with updated plug-ins) and Imaris. Seahorse data were analyzed using WAVE Software. NAD/NADH-GIo ${ }^{\mathrm{TM}}$ bioluminescent Assay were measured by CLARIOstar plate reader. GraphPad Prism 9.0 (GraphPad Software) was used for statistical analysis. The normality of residuals was checked by Kolmogorov-Smirnov test. For normally distributed residuals, one-way ANOVA, two-way ANOVA, or unpaired student's t-test was used. For data that did not pass the Kolmogorov-Smirnov test, one-way ANOVA was replaced by 
Kruskal-Wallis test; two-way ANOVA was replaced by Multiple Mann-Whitney test or simplified to use the Kruskal-Wallis test; unpaired student's t-test was replaced by the Mann Whitney test.

To analyze cumulative frequency distribution curve, the dependent variable $\mathrm{P}$ (the relative percentage within each bin) was transformed into $\log (\mathrm{P} /(100-\mathrm{P}))$ so that the relationship between bin of sphericity (covariate) and the dependent variable was linearized. Then, a linear mixed model with random slope and intercept was generated by SPSS using a restricted maximum likelihood approach to compare statistical difference between control and $\mathrm{KO}$. Criterion for statistical significance was set at $p<0.05$ for all statistical analyses.

\section{Reagents}

\begin{tabular}{|c|c|c|}
\hline Reagents & Source & Identifier \\
\hline \multicolumn{3}{|l|}{ Antibodies } \\
\hline $\begin{array}{l}\text { Rabbit monoclonal anti-APP } \\
\text { (1:1000 for IHC \& ICC) }\end{array}$ & Abcam & $\begin{array}{l}\text { Abcam Cat\# ab32136, } \\
\text { RRID:AB_2289606 }\end{array}$ \\
\hline $\begin{array}{l}\text { Mouse monoclonal Anti-beta-Tubulin III } \\
(1: 1000 \text { to } 1: 2000 \text { for ICC) }\end{array}$ & Millipore & $\begin{array}{l}\text { Millipore Cat\# 05-559, } \\
\text { RRID:AB_309804 }\end{array}$ \\
\hline $\begin{array}{l}\text { Chicken polyclonal anti-MAP2 } \\
(1: 1000 \text { to } 1: 2000 \text { for ICC) }\end{array}$ & Millipore & $\begin{array}{l}\text { Millipore Cat\# AB5543, RRID: } \\
\text { AB_571049 }\end{array}$ \\
\hline $\begin{array}{l}\text { Rabbit polyclonal anti-PSD95 } \\
(1: 1000 \text { for WB) }\end{array}$ & $\begin{array}{l}\text { Invitrogen } \\
\text { (Original Zymed) }\end{array}$ & $\begin{array}{l}\text { Thermo Fisher Scientific Cat\# } \\
\text { 51-6900, RRID:AB_2533914 }\end{array}$ \\
\hline $\begin{array}{l}\text { Rabbit polyclonal anti-SNAP25 } \\
(1: 1000 \text { for WB) }\end{array}$ & SYSY & $\begin{array}{l}\text { Synaptic Systems Cat\# } 111 \\
\text { 002, RRID:AB_887790 }\end{array}$ \\
\hline $\begin{array}{l}\text { Rabbit polyclonal anti-Synapsin } 1 / 2 \\
(1: 1000 \text { for WB) }\end{array}$ & SYSY & $\begin{array}{l}\text { Synaptic Systems Cat\# } 106 \\
\text { 002, RRID:AB_887804 }\end{array}$ \\
\hline $\begin{array}{l}\text { Mouse monoclonal anti-GAPDH } \\
(1: 2000 \text { for WB) }\end{array}$ & Millipore & $\begin{array}{l}\text { Millipore Cat\# MAB374, } \\
\text { RRID:AB_2107445 }\end{array}$ \\
\hline $\begin{array}{l}\text { Rabbit polyclonal anti-RFP } \\
(1: 1000 \text { for ICC) }\end{array}$ & Rockland & $\begin{array}{l}\text { Rockland Cat\# 600-401-379, } \\
\text { RRID:AB_2209751 }\end{array}$ \\
\hline $\begin{array}{l}\text { Chicken polyclonal anti-GFP } \\
(1: 1000 \text { for ICC) }\end{array}$ & Aves Labs & $\begin{array}{l}\text { Aves Labs Cat\# GFP-1010, } \\
\text { RRID:AB_2307313 }\end{array}$ \\
\hline $\begin{array}{l}\text { Rat monoclonal anti-HA tag } \\
(1: 500 \text { for ICC; } 1: 1000 \text { for WB) }\end{array}$ & Roche & $\begin{array}{l}\text { Roche Cat\# } 11867423001, \\
\text { RRID:AB_390918 }\end{array}$ \\
\hline $\begin{array}{l}\text { Rabbit polyclonal anti-Actin } \\
(1: 2000 \text { for WB) }\end{array}$ & Sigma-Aldrich & $\begin{array}{l}\text { Sigma-Aldrich Cat\# A2066, } \\
\text { RRID:AB_476693 }\end{array}$ \\
\hline
\end{tabular}




\begin{tabular}{|c|c|c|}
\hline $\begin{array}{l}\text { Goat anti-Rabbit IgG }(\mathrm{H}+\mathrm{L}) \text {, Alexa Fluor } 555 \\
\text { Conjugated } \\
(1: 1000 \text { for IHC \& ICC) }\end{array}$ & Molecular Probes & $\begin{array}{l}\text { Molecular Probes Cat\# A- } \\
\text { 21429, RRID:AB_2535850 }\end{array}$ \\
\hline $\begin{array}{l}\text { Goat anti-Mouse } \lg G(\mathrm{H}+\mathrm{L}) \text {, Alexa Fluor } 488 \\
\text { Conjugated } \\
(1: 1000 \text { for ICC })\end{array}$ & Molecular Probes & $\begin{array}{l}\text { Molecular Probes Cat\# A- } \\
\text { 11029, RRID:AB_138404 }\end{array}$ \\
\hline $\begin{array}{l}\text { Goat anti-Chicken IgG }(H+L) \text {, Alexa Fluor } 647 \\
\text { Conjugated } \\
(1: 1000 \text { for ICC) }\end{array}$ & $\begin{array}{l}\text { Innovative } \\
\text { Research }\end{array}$ & $\begin{array}{l}\text { Innovative Research Cat\# } \\
\text { A21449, RRID:AB_1500594 }\end{array}$ \\
\hline $\begin{array}{l}\text { Goat anti-Rat IgG }(H+L) \text {, Alexa Fluor } 647 \\
\text { Conjugated } \\
(1: 1000 \text { for ICC })\end{array}$ & Invitrogen & $\begin{array}{l}\text { Molecular Probes Cat\# A- } \\
\text { 21247, RRID:AB_141778 }\end{array}$ \\
\hline $\begin{array}{l}\text { Goat anti-Rabbit, IRDye } \AA 800 C W \text { Conjugated } \\
\text { (1:5000 for WB) }\end{array}$ & LI-COR & $\begin{array}{l}\text { LI-COR Biosciences Cat\# 926- } \\
\text { 32211, RRID:AB_621843 }\end{array}$ \\
\hline $\begin{array}{l}\text { Goat anti-Rabbit, IRDye } \AA \text { 680LT Conjugated } \\
\text { (1:5000 for WB) }\end{array}$ & LI-COR & $\begin{array}{l}\text { LI-COR Biosciences Cat\# 926- } \\
\text { 68021, RRID:AB_10706309 }\end{array}$ \\
\hline $\begin{array}{l}\text { Goat anti-Mouse, IRDye }{ }^{\circledR} 800 \mathrm{CW} \text { Conjugated } \\
\text { (1:5000 for WB) }\end{array}$ & LI-COR & $\begin{array}{l}\text { LI-COR Biosciences Cat\# 926- } \\
\text { 32210, RRID:AB_621842 }\end{array}$ \\
\hline $\begin{array}{l}\text { Goat anti-Mouse, IRDye® } 680 \text { LT Conjugated } \\
\text { (1:5000 for WB) }\end{array}$ & LI-COR & $\begin{array}{l}\text { LI-COR Biosciences Cat\# 827- } \\
\text { 11080, RRID:AB_10795014 }\end{array}$ \\
\hline $\begin{array}{l}\text { Goat anti-Rat, IRDye® 800CW Conjugated } \\
\text { (1:5000 for WB) }\end{array}$ & LI-COR & $\begin{array}{l}\text { LI-COR Biosciences Cat\# 925- } \\
\text { 32219, RRID:AB_2721932 }\end{array}$ \\
\hline Draq5 & Cell Signaling & $4084 \mathrm{~L}$ \\
\hline DAPI & Invitrogen & D3571 \\
\hline ProLong ${ }^{\mathrm{TM}}$ Gold Antifade Mountant with DAPI & Invitrogen & P36931 \\
\hline Dako Mounting Medium & $\begin{array}{l}\text { Agilent } \\
\text { Technologies }\end{array}$ & CS70330-2 \\
\hline \multicolumn{3}{|l|}{ Bacterial and virus strains } \\
\hline Stbl3 competent E. coli & ThermoFisher & C737303 \\
\hline LV-ELF1alpha-copGFP & Baylor vector core & \\
\hline LV-ELF1alpha-(WT)Nmnat2-IRES-copGFP & Baylor vector core & \\
\hline LV-ELF1alpha-(PM)Nmnat2-IRES-copGFP & Baylor vector core & \\
\hline \multicolumn{3}{|l|}{ Chemicals, peptides, and recombinant proteins } \\
\hline Oligomycin & Calbiochem & 495455 \\
\hline Oligomycin & Sigma-Aldrich & O4876 \\
\hline 2-Deoxy-D-glucose & Sigma-Aldrich & D6134 \\
\hline
\end{tabular}




\begin{tabular}{|c|c|c|}
\hline Methyl pyruvate & $\begin{array}{l}\text { ACROS } \\
\text { Organics }^{\mathrm{TM}}\end{array}$ & AC127650250 \\
\hline Sodium L-lactate & Sigma-Aldrich & 71718 \\
\hline NAD & Roche & NAD100-RO \\
\hline B-Nicotinamide Mononucleotide & Cayman & 16411 \\
\hline Nicotinamide & $\begin{array}{l}\text { ACROS } \\
\text { Organics }^{\mathrm{TM}}\end{array}$ & AC128271000 \\
\hline Hibernate E Low Fluorescence & BrainBits & HELF \\
\hline Hibernate E Low Fluorescence-Glucose free & BrainBits & Customized \\
\hline Seahorse XF DMEM medium & Agilent & $103575-100$ \\
\hline Seahorse XF Calibrant Solution & Agilent & $100840-000$ \\
\hline Seahorse XF 200 mM glutamine solution & Agilent & $103579-100$ \\
\hline Seahorse XF 100 mM pyruvate solution & Agilent & $103578-100$ \\
\hline Seahorse XF 1.0 M glucose solution & Agilent & $103577-100$ \\
\hline N-Methyl-D-aspartic acid & Sigma-Aldrich & M3262-25MG \\
\hline Glycine & MP Biomedical & 02194825-CF \\
\hline Lipofectamine $^{\mathrm{TM}} 3000$ Transfection Reagent & $\begin{array}{l}\text { Thermo Fisher } \\
\text { Scientific }\end{array}$ & L3000008 \\
\hline CombiMag & OZ Biosciences & CM20100 \\
\hline Lipofectamine $^{\mathrm{TM}} 2000$ Transfection Reagent & $\begin{array}{l}\text { Thermo Fisher } \\
\text { Scientific }\end{array}$ & 11668030 \\
\hline Papain Dissociation System & $\begin{array}{l}\text { Worthington } \\
\text { Biochem Co. }\end{array}$ & LK003150 \\
\hline Proteinase K, recombinant, PCR Grade & Roche & RPROTKSOL-RO \\
\hline EconoTaq ${ }^{\circledR}$ PLUS 2X PCR Master Mix & Lucigen & $95024-006$ \\
\hline QIAGEN Fast Cycling PCR Kit & Qiagen & 203743 \\
\hline Poly-D-lysine hydrobromide & Sigma-Aldrich & P6407 \\
\hline B27 supplement & $\begin{array}{l}\text { Thermo Fisher } \\
\text { Scientific }\end{array}$ & 17504044 \\
\hline GlutaMAX ${ }^{\mathrm{TM}}$ Supplement & $\begin{array}{l}\text { Thermo Fisher } \\
\text { Scientific }\end{array}$ & 35050061 \\
\hline Penicillin-Streptomycin $(10,000 \mathrm{U} / \mathrm{mL})$ & $\begin{array}{l}\text { Thermo Fisher } \\
\text { Scientific }\end{array}$ & 15140122 \\
\hline Neurobasal medium & $\begin{array}{l}\text { Thermo Fisher } \\
\text { Scientific }\end{array}$ & 21103049 \\
\hline $\begin{array}{l}\text { cOmplete }{ }^{\mathrm{TM}} \text {, Mini, EDTA-free Protease Inhibitor } \\
\text { Cocktail }\end{array}$ & Roche & 11836170001 \\
\hline
\end{tabular}




\begin{tabular}{|c|c|c|}
\hline Dodecyltrimethylammonium bromide & Sigma-Aldrich & D8638 \\
\hline $\begin{array}{l}\text { Bio-Rad Protein Assay Dye Reagent } \\
\text { Concentrate }\end{array}$ & Bio-Rad & \#5000006 \\
\hline Pierce BCA Protein Assay Kit & Thermo Fisher & 23225 \\
\hline QIAGEN Plasmid Plus Maxi Kit & Qiagen & 12963 \\
\hline RNeasy Mini Kit & Qiagen & 74104 \\
\hline \multicolumn{3}{|l|}{ Critical commercial assays } \\
\hline NAD/NADH-Glo ${ }^{\text {TM }}$ Assay & Promega & G9071 \\
\hline Seahorse XF Glycolysis Rate Assay Kit & Agilent & $103344-100$ \\
\hline Seahorse XF Mito Stress Test Kit & Agilent & $103015-100$ \\
\hline \multicolumn{3}{|l|}{ Experimental models: Organisms/strains } \\
\hline Transgenic mice: NMNAT2-BLAD & $(41)$ & FVB background \\
\hline Transgenic mice: NMNAT2 ${ }^{\mathrm{t} / \mathrm{f}}$ & $(79)$ & C57BL/6J background \\
\hline Transgenic mice: NEX-Cre & (32) & C57BL/6 background \\
\hline \multicolumn{3}{|l|}{ Oligonucleotides } \\
\hline $\begin{array}{l}\text { Primer for NMNAT2-BLAD genotyping R3: } \\
\text { 5' - CCACTGGGA ATG TGA TGA AAG AAA } \\
\text { TAA AAG C -3' }\end{array}$ & This paper & \\
\hline $\begin{array}{l}\text { Primer for NMNAT2-BLAD genotyping RF: } \\
\text { 5' -CTG ACG TCT ATC TAG AAG TAC AC -3' }\end{array}$ & This paper & \\
\hline $\begin{array}{l}\text { Primer for NMNAT2-BLAD genotyping UP: } \\
\text { 5'- GGA AAA GCC CAA ATCCCA GCA A -3' }\end{array}$ & This paper & \\
\hline $\begin{array}{l}\text { Primer for NMNAT2 }{ }^{\mathrm{f} / \mathrm{f}} \text { genotyping A: } \\
\text { 5'- GCT GGC CTA GGT GGT GAT TTGC -3' }\end{array}$ & This paper & \\
\hline $\begin{array}{l}\text { Primer for NMNAT2/f/ genotyping B: } \\
\text { 5'- ACT GGG ATG GCA CGA GAC CCT GC -3' }\end{array}$ & This paper & \\
\hline $\begin{array}{l}\text { Primer for NMNAT2 }{ }^{\mathrm{t} / \mathrm{f}} \text { genotyping C: } \\
\text { 5'- AGT CAT AGA CAC TAG ACA ATCG -3' }\end{array}$ & This paper & \\
\hline $\begin{array}{l}\text { Primer for NEX-Cre genotyping Cre484: } \\
\text { 5'- GCATTTCTGGGGATTGCTTA -3' }\end{array}$ & This paper & \\
\hline $\begin{array}{l}\text { Primer for NEX-Cre genotyping Cre834: } \\
\text { 5'-GTCATCCTTAGCGCCGTAAA -3' }\end{array}$ & This paper & \\
\hline \multicolumn{3}{|l|}{ Recombinant DNA } \\
\hline pEGFP-n1-APP & $(80)$ & Addgene Plasmid \#69924 \\
\hline pEGFP-c1-SNAP25 & $(27)$ & \\
\hline pmCherry-n1-NMNAT2 & $(27)$ & \\
\hline
\end{tabular}




\begin{tabular}{|c|c|c|}
\hline pLV-mitoDsRed & $(82)$ & Addgene plasmid \# 44386 \\
\hline pCX-EGFP & $(81)$ & \\
\hline pcDNA3.1- SoNar & $(52)$ & \\
\hline pcDNA3.1-cpYFP & $(52)$ & \\
\hline pCAG-mCherry & $\begin{array}{l}\text { Gift from Ken } \\
\text { Mackie }\end{array}$ & \\
\hline pCMV-MitoVenus & $(26)$ & \\
\hline pcDNA3-Syn-ATP & (53) & Addgene plasmid \#51819 \\
\hline pcDNA3-Cyto-pHluorin & (53) & \\
\hline \multicolumn{3}{|l|}{ Software and algorithms } \\
\hline Fiji (Image J with updated plug-in) & $\mathrm{NIH}$ & https://fiji.sc/ \\
\hline Imaris & OXFORD & https://imaris.oxinst.com/ \\
\hline GraphPad Prism 9.0 & GraphPad & https://www.graphpad.com/ \\
\hline WAVE & Agilent & $\begin{array}{l}\text { https://www.agilent.com/en/prod } \\
\text { ucts/cell-analysis/software- } \\
\text { download-for-wave-desktop }\end{array}$ \\
\hline SPSS & IBM & $\begin{array}{l}\text { https://www.ibm.com/analytics/s } \\
\text { pss-statistics-software }\end{array}$ \\
\hline \multicolumn{3}{|l|}{ Other } \\
\hline Round cover glass, \#1.5 thickness, $12 \mathrm{~mm}$ & Thomas Scientific & 1217 N79 \\
\hline Seahorse XFe24 FluxPak & Agilent & $102340-100$ \\
\hline $\begin{array}{l}35 \mathrm{~mm} \text { Dish | No. } 1.5 \text { Coverslip | } 20 \text { mm Glass } \\
\text { Diameter | Uncoated }\end{array}$ & MaTek & P35G-1.5-20-C \\
\hline $\begin{array}{l}35 \text { mm Dish | No. } 1.5 \text { Coverslip | } 14 \text { mm Glass } \\
\text { Diameter | Uncoated }\end{array}$ & MaTek & P35G-1.5-14-C \\
\hline
\end{tabular}




\section{Reference}

1. J. Winnubst, E. Bas, T. A. Ferreira, Z. Wu, M. N. Economo, P. Edson, B. J. Arthur, C. Bruns, K. Rokicki, D. Schauder, D. J. Olbris, S. D. Murphy, D. G. Ackerman, C. Arshadi, P. Baldwin, R. Blake, A. Elsayed, M. Hasan, D. Ramirez, B. Dos Santos, M. Weldon, A. Zafar, J. T. Dudman, C. R. Gerfen, A. W. Hantman, W. Korff, S. M. Sternson, N. Spruston, K. Svoboda, J. Chandrashekar, Reconstruction of 1,000 Projection Neurons Reveals New Cell Types and Organization of LongRange Connectivity in the Mouse Brain. Cell 179, 268-281 e213 (2019).

2. J. N. Sleigh, A. M. Rossor, A. D. Fellows, A. P. Tosolini, G. Schiavo, Axonal transport and neurological disease. Nat Rev Neurol 15, 691-703 (2019).

3. C. F. Ibanez, Message in a bottle: long-range retrograde signaling in the nervous system. Trends Cell Biol 17, 519-528 (2007).

4. P. Guedes-Dias, E. L. F. Holzbaur, Axonal transport: Driving synaptic function. Science 366, (2019).

5. C. Pacelli, N. Giguere, M. J. Bourque, M. Levesque, R. S. Slack, L. E. Trudeau, Elevated Mitochondrial Bioenergetics and Axonal Arborization Size Are Key Contributors to the Vulnerability of Dopamine Neurons. Curr Bio/ 25, 2349-2360 (2015).

6. G. Gallo, The bioenergetics of neuronal morphogenesis and regeneration: Frontiers beyond the mitochondrion. Dev Neurobiol 80, 263-276 (2020).

7. K. A. Chamberlain, Z. H. Sheng, Mechanisms for the maintenance and regulation of axonal energy supply. J Neurosci Res 97, 897-913 (2019).

8. L. R. Fischer, D. G. Culver, P. Tennant, A. A. Davis, M. Wang, A. Castellano-Sanchez, J. Khan, M. A. Polak, J. D. Glass, Amyotrophic lateral sclerosis is a distal axonopathy: evidence in mice and man. Exp Neurol 185, 232-240 (2004).

9. A. W. Xiao, J. He, Q. Wang, Y. Luo, Y. Sun, Y. P. Zhou, Y. Guan, P. J. Lucassen, J. P. Dai, The origin and development of plaques and phosphorylated tau are associated with axonopathy in Alzheimer's disease. Neurosci Bull 27, 287-299 (2011).

10. G. B. Stokin, C. Lillo, T. L. Falzone, R. G. Brusch, E. Rockenstein, S. L. Mount, R. Raman, P. Davies, E. Masliah, D. S. Williams, L. S. Goldstein, Axonopathy and transport deficits early in the pathogenesis of Alzheimer's disease. Science 307, 1282-1288 (2005).

11. M. P. Coleman, A. Hoke, Programmed axon degeneration: from mouse to mechanism to medicine. Nat Rev Neurosci 21, 183-196 (2020). 
12. J. Gerdts, D. W. Summers, J. Milbrandt, A. DiAntonio, Axon Self-Destruction: New Links among SARM1, MAPKs, and NAD+ Metabolism. Neuron 89, 449-460 (2016).

13. D. J. Simon, T. A. Watkins, Therapeutic opportunities and pitfalls in the treatment of axon degeneration. Curr Opin Neurol 31, 693-701 (2018).

14. F. Berger, C. Lau, M. Dahlmann, M. Ziegler, Subcellular compartmentation and differential catalytic properties of the three human nicotinamide mononucleotide adenylyltransferase isoforms. J Biol Chem 280, 36334-36341 (2005).

15. N. Raffaelli, L. Sorci, A. Amici, M. Emanuelli, F. Mazzola, G. Magni, Identification of a novel human nicotinamide mononucleotide adenylyltransferase. Biochem Biophys Res Commun 297, 835-840 (2002).

16. T. Yan, Y. Feng, J. Zheng, X. Ge, Y. Zhang, D. Wu, J. Zhao, Q. Zhai, Nmnat2 delays axon degeneration in superior cervical ganglia dependent on its NAD synthesis activity. Neurochem Int 56, 101-106 (2010).

17. Y. O. Ali, H. M. Allen, L. Yu, D. Li-Kroeger, D. Bakhshizadehmahmoudi, A. Hatcher, C. McCabe, J. Xu, N. Bjorklund, G. Taglialatela, D. A. Bennett, P. L. De Jager, J. M. Shulman, H. J. Bellen, H. C. Lu, NMNAT2:HSP90 Complex Mediates Proteostasis in Proteinopathies. PLoS Biol 14, e1002472 (2016).

18. J. P. Bennett, P. M. Keeney, RNA-Sequencing Reveals Similarities and Differences in Gene Expression in Vulnerable Brain Tissues of Alzheimer's and Parkinson's Diseases. J Alzheimers Dis Rep 2, 129-137 (2018).

19. Y. O. Ali, D. Li-Kroeger, H. J. Bellen, R. G. Zhai, H. C. Lu, NMNATs, evolutionarily conserved neuronal maintenance factors. Trends Neurosci 36, 632-640 (2013).

20. B. A. Harlan, K. M. Killoy, M. Pehar, L. Liu, J. Auwerx, M. R. Vargas, Evaluation of the NAD(+) biosynthetic pathway in ALS patients and effect of modulating NAD(+) levels in hSOD1-linked ALS mouse models. Exp Neurol 327, 113219 (2020).

21. J. Gilley, M. P. Coleman, Endogenous Nmnat2 is an essential survival factor for maintenance of healthy axons. PLoS Biol 8, e1000300 (2010).

22. A. Loreto, C. S. Hill, V. L. Hewitt, G. Orsomando, C. Angeletti, J. Gilley, C. Lucci, A. SanchezMartinez, A. J. Whitworth, L. Conforti, F. Dajas-Bailador, M. P. Coleman, Mitochondrial impairment activates the Wallerian pathway through depletion of NMNAT2 leading to SARM1dependent axon degeneration. Neurobiol Dis 134, 104678 (2020). 
23. J. M. Brazill, C. Li, Y. Zhu, R. G. Zhai, NMNAT: It's an NAD(+) synthase... It's a chaperone... It's a neuroprotector. Curr Opin Genet Dev 44, 156-162 (2017).

24. E. Katsyuba, M. Romani, D. Hofer, J. Auwerx, NAD(+) homeostasis in health and disease. Nat Metab 2, 9-31 (2020).

25. N. Xie, L. Zhang, W. Gao, C. Huang, P. E. Huber, X. Zhou, C. Li, G. Shen, B. Zou, NAD ${ }^{+}$metabolism: pathophysiologic mechanisms and therapeutic potential. Signal Transduct Target Ther 5, 227 (2020).

26. X. A. Cambronne, M. L. Stewart, D. Kim, A. M. Jones-Brunette, R. K. Morgan, D. L. Farrens, M. S. Cohen, R. H. Goodman, Biosensor reveals multiple sources for mitochondrial NAD(+). Science 352, 1474-1477 (2016).

27. S. Milde, J. Gilley, M. P. Coleman, Subcellular localization determines the stability and axon protective capacity of axon survival factor Nmnat2. PLoS Biol 11, e1001539 (2013).

28. P. R. Mayer, N. Huang, C. M. Dewey, D. R. Dries, H. Zhang, G. Yu, Expression, localization, and biochemical characterization of nicotinamide mononucleotide adenylyltransferase 2. J Biol Chem 285, 40387-40396 (2010).

29. C. O. Hung, M. P. Coleman, KIF1A mediates axonal transport of BACE1 and identification of independently moving cargoes in living SCG neurons. Traffic 17, 1155-1167 (2016).

30. D. Zala, M. V. Hinckelmann, H. Yu, M. M. Lyra da Cunha, G. Liot, F. P. Cordelieres, S. Marco, F. Saudou, Vesicular glycolysis provides on-board energy for fast axonal transport. Cell 152, 479491 (2013).

31. M. V. Hinckelmann, A. Virlogeux, C. Niehage, C. Poujol, D. Choquet, B. Hoflack, D. Zala, F. Saudou, Self-propelling vesicles define glycolysis as the minimal energy machinery for neuronal transport. Nat Commun 7, 13233 (2016).

32. S. Goebbels, I. Bormuth, U. Bode, O. Hermanson, M. H. Schwab, K. A. Nave, Genetic targeting of principal neurons in neocortex and hippocampus of NEX-Cre mice. Genesis 44, 611-621 (2006).

33. S. Jawhar, A. Trawicka, C. Jenneckens, T. A. Bayer, O. Wirths, Motor deficits, neuron loss, and reduced anxiety coinciding with axonal degeneration and intraneuronal Abeta aggregation in the 5XFAD mouse model of Alzheimer's disease. Neurobiol Aging 33, 196 e129-140 (2012).

34. D. De Silva, S. Hsieh, J. Caga, F. V. Leslie, M. C. Kiernan, J. R. Hodges, E. Mioshi, J. R. Burrell, Motor function and behaviour across the ALS-FTD spectrum. Acta Neurol Scand 133, 367-372 (2016). 
35. Y. Liu, A. Pattamatta, T. Zu, T. Reid, O. Bardhi, D. R. Borchelt, A. T. Yachnis, L. P. Ranum, C9orf72 BAC Mouse Model with Motor Deficits and Neurodegenerative Features of ALS/FTD. Neuron 90, 521-534 (2016).

36. M. M. Fu, E. L. Holzbaur, JIP1 regulates the directionality of APP axonal transport by coordinating kinesin and dynein motors. J Cell Biol 202, 495-508 (2013).

37. U. C. Muller, T. Deller, M. Korte, Not just amyloid: physiological functions of the amyloid precursor protein family. Nat Rev Neurosci 18, 281-298 (2017).

38. O. Wirths, J. Weis, R. Kayed, T. C. Saido, T. A. Bayer, Age-dependent axonal degeneration in an Alzheimer mouse model. Neurobiol Aging 28, 1689-1699 (2007).

39. T. Hortobagyi, S. Wise, N. Hunt, N. Cary, V. Djurovic, A. Fegan-Earl, K. Shorrock, D. Rouse, S. AlSarraj, Traumatic axonal damage in the brain can be detected using beta-APP immunohistochemistry within 35 min after head injury to human adults. Neuropathol Appl Neurobiol 33, 226-237 (2007).

40. S. Ruhling, F. Kramer, S. Schmutz, S. Amor, Z. Jiangshan, C. Schmitz, M. Kipp, T. Hochstrasser, Visualization of the Breakdown of the Axonal Transport Machinery: a Comparative Ultrastructural and Immunohistochemical Approach. Mol Neurobio/ 56, 3984-3998 (2019).

41. A. N. Hicks, D. Lorenzetti, J. Gilley, B. Lu, K. E. Andersson, C. Miligan, P. A. Overbeek, R. Oppenheim, C. E. Bishop, Nicotinamide mononucleotide adenylyltransferase 2 (Nmnat2) regulates axon integrity in the mouse embryo. PLoS One 7, e47869 (2012).

42. A. F. Ikin, W. G. Annaert, K. Takei, P. De Camilli, R. Jahn, P. Greengard, J. D. Buxbaum, Alzheimer amyloid protein precursor is localized in nerve terminal preparations to Rab5-containing vesicular organelles distinct from those implicated in the synaptic vesicle pathway. J Biol Chem 271, 31783-31786 (1996).

43. M. Shapira, R. G. Zhai, T. Dresbach, T. Bresler, V. I. Torres, E. D. Gundelfinger, N. E. Ziv, C. C. Garner, Unitary assembly of presynaptic active zones from Piccolo-Bassoon transport vesicles. Neuron 38, 237-252 (2003).

44. K. Chiba, M. Araseki, K. Nozawa, K. Furukori, Y. Araki, T. Matsushima, T. Nakaya, S. Hata, Y. Saito, S. Uchida, Y. Okada, A. C. Nairn, R. J. Davis, T. Yamamoto, M. Kinjo, H. Taru, T. Suzuki, Quantitative analysis of APP axonal transport in neurons: role of JIP1 in enhanced APP anterograde transport. Mol Biol Cell 25, 3569-3580 (2014).

45. G. Shiff, N. Morel, Rapid anterograde axonal transport of the syntaxin-SNAP 25-VAMP complex. J Neurochem 68, 1663-1667 (1997). 
46. Q. Cai, P. Y. Pan, Z. H. Sheng, Syntabulin-kinesin-1 family member 5B-mediated axonal transport contributes to activity-dependent presynaptic assembly. J Neurosci 27, 7284-7296 (2007).

47. I. R. Boldogh, L. A. Pon, Mitochondria on the move. Trends Cell Biol 17, 502-510 (2007).

48. A. Melkov, U. Abdu, Regulation of long-distance transport of mitochondria along microtubules. Cell Mol Life Sci 75, 163-176 (2018).

49. G. Plucinska, T. Misgeld, Imaging of neuronal mitochondria in situ. Curr Opin Neurobiol 39, 152163 (2016).

50. S. Lautrup, D. A. Sinclair, M. P. Mattson, E. F. Fang, NAD(+) in Brain Aging and Neurodegenerative Disorders. Cell Metab 30, 630-655 (2019).

51. Y. Sasaki, Metabolic aspects of neuronal degeneration: From a NAD(+) point of view. Neurosci Res 139, 9-20 (2019).

52. Y. Zhao, Q. Hu, F. Cheng, N. Su, A. Wang, Y. Zou, H. Hu, X. Chen, H. M. Zhou, X. Huang, K. Yang, Q. Zhu, X. Wang, J. Yi, L. Zhu, X. Qian, L. Chen, Y. Tang, J. Loscalzo, Y. Yang, SoNar, a Highly Responsive NAD+/NADH Sensor, Allows High-Throughput Metabolic Screening of Anti-tumor Agents. Cell Metab 21, 777-789 (2015).

53. V. Rangaraju, N. Calloway, T. A. Ryan, Activity-driven local ATP synthesis is required for synaptic function. Cell 156, 825-835 (2014).

54. S. C. Cunnane, E. Trushina, C. Morland, A. Prigione, G. Casadesus, Z. B. Andrews, M. F. Beal, L. H. Bergersen, R. D. Brinton, S. de la Monte, A. Eckert, J. Harvey, R. Jeggo, J. H. Jhamandas, O. Kann, C. M. la Cour, W. F. Martin, G. Mithieux, P. I. Moreira, M. P. Murphy, K. A. Nave, T. Nuriel, S. H. R. Oliet, F. Saudou, M. P. Mattson, R. H. Swerdlow, M. J. Millan, Brain energy rescue: an emerging therapeutic concept for neurodegenerative disorders of ageing. Nat Rev Drug Discov 19, 609-633 (2020).

55. C. C. Alano, P. Garnier, W. Ying, Y. Higashi, T. M. Kauppinen, R. A. Swanson, NAD+ depletion is necessary and sufficient for poly(ADP-ribose) polymerase-1-mediated neuronal death. $J$ Neurosci 30, 2967-2978 (2010).

56. S. Wang, Z. Xing, P. S. Vosler, H. Yin, W. Li, F. Zhang, A. P. Signore, R. A. Stetler, Y. Gao, J. Chen, Cellular NAD replenishment confers marked neuroprotection against ischemic cell death: role of enhanced DNA repair. Stroke 39, 2587-2595 (2008).

57. R. G. Zhai, Y. Cao, P. R. Hiesinger, Y. Zhou, S. Q. Mehta, K. L. Schulze, P. Verstreken, H. J. Bellen, Drosophila NMNAT maintains neural integrity independent of its NAD synthesis activity. PLoS Biol 4, e416 (2006). 
58. I. Perez-Liebana, I. Juaristi, P. González-Sánchez, L. González-Moreno, E. Rial, M. Podunavac, A. Zakarian, J. Molgó, B. Pardo, J. Satrustegui, A feed-forward Ca2+-dependent mechanism boosting glycolysis and OXPHOS by activating Aralar-malate-aspartate shuttle, upon neuronal stimulation. bioRxiv, (2021).

59. X. Zhou, D. Hollern, J. Liao, E. Andrechek, H. Wang, NMDA receptor-mediated excitotoxicity depends on the coactivation of synaptic and extrasynaptic receptors. Cell Death Dis 4, e560 (2013).

60. D. W. Summers, A. DiAntonio, J. Milbrandt, Mitochondrial dysfunction induces Sarm1dependent cell death in sensory neurons. J Neurosci 34, 9338-9350 (2014).

61. M. D. Figley, W. Gu, J. D. Nanson, Y. Shi, Y. Sasaki, K. Cunnea, A. K. Malde, X. Jia, Z. Luo, F. K. Saikot, T. Mosaiab, V. Masic, S. Holt, L. Hartley-Tassell, H. Y. McGuinness, M. K. Manik, T. Bosanac, M. J. Landsberg, P. S. Kerry, M. Mobli, R. O. Hughes, J. Milbrandt, B. Kobe, A. DiAntonio, T. Ve, SARM1 is a metabolic sensor activated by an increased NMN/NAD(+) ratio to trigger axon degeneration. Neuron 109, 1118-1136 e1111 (2021).

62. A. Izadifar, J. Courchet, D. M. Virga, T. Verreet, S. Hamilton, D. Ayaz, A. Misbaer, S. Vandenbogaerde, L. Monteiro, M. Petrovic, S. Sachse, B. Yan, M. L. Erfurth, D. Dascenco, Y. Kise, J. Yan, G. Edwards-Faret, T. Lewis, F. Polleux, D. Schmucker, Axon morphogenesis and maintenance require an evolutionary conserved safeguard function of Wnk kinases antagonizing Sarm and Axed. Neuron 109, 2864-2883 e2868 (2021).

63. Y. Zilberter, M. Zilberter, The vicious circle of hypometabolism in neurodegenerative diseases: Ways and mechanisms of metabolic correction. J Neurosci Res 95, 2217-2235 (2017).

64. N. S. Chandel, Glycolysis. Cold Spring Harb Perspect Biol 13, (2021).

65. T. L. Lewis, Jr., S. K. Kwon, A. Lee, R. Shaw, F. Polleux, MFF-dependent mitochondrial fission regulates presynaptic release and axon branching by limiting axonal mitochondria size. Nat Commun 9, 5008 (2018).

66. T. L. Lewis, Jr., G. F. Turi, S. K. Kwon, A. Losonczy, F. Polleux, Progressive Decrease of Mitochondrial Motility during Maturation of Cortical Axons In Vitro and In Vivo. Curr Biol 26, 2602-2608 (2016).

67. E. Moutaux, W. Christaller, C. Scaramuzzino, A. Genoux, B. Charlot, M. Cazorla, F. Saudou, Neuronal network maturation differently affects secretory vesicles and mitochondria transport in axons. Sci Rep 8, 13429 (2018). 
68. B. Zhou, P. Yu, M. Y. Lin, T. Sun, Y. Chen, Z. H. Sheng, Facilitation of axon regeneration by enhancing mitochondrial transport and rescuing energy deficits. J Cell Biol 214, 103-119 (2016).

69. A. D. de Lima, M. D. Merten, T. Voigt, Neuritic differentiation and synaptogenesis in serum-free neuronal cultures of the rat cerebral cortex. J Comp Neurol 382, 230-246 (1997).

70. G. Yellen, Fueling thought: Management of glycolysis and oxidative phosphorylation in neuronal metabolism. J Cell Biol 217, 2235-2246 (2018).

71. M. Mc cluskey, thesis, "'Study of vesicular glycolysis in health and Huntington's Disease"', thesis, Université Grenoble Alpes [2020-....] (2021).

72. M. J. Schnitzer, S. M. Block, Kinesin hydrolyses one ATP per 8-nm step. Nature 388, 386-390 (1997).

73. J. Niu, S. S. Sanders, H. K. Jeong, S. M. Holland, Y. Sun, K. M. Collura, L. M. Hernandez, H. Huang, M. R. Hayden, G. M. Smith, Y. Hu, Y. Jin, G. M. Thomas, Coupled Control of Distal Axon Integrity and Somal Responses to Axonal Damage by the Palmitoyl Acyltransferase ZDHHC17. Cell Rep 33, 108365 (2020).

74. A. Virlogeux, C. Scaramuzzino, S. Lenoir, R. Carpentier, M. Louessard, A. Genoux, P. Lino, M. V. Hinckelmann, A. L. Perrier, S. Humbert, F. Saudou, Increasing brain palmitoylation rescues behavior and neuropathology in Huntington disease mice. Sci Adv 7, (2021).

75. N. L. Turner, T. Macrina, J. A. Bae, R. Yang, A. M. Wilson, C. Schneider-Mizell, K. Lee, R. Lu, J. Wu, A. L. Bodor, A. A. Bleckert, D. Brittain, E. Froudarakis, S. Dorkenwald, F. Collman, N. Kemnitz, D. Ih, W. M. Silversmith, J. Zung, A. Zlateski, I. Tartavull, S.-c. Yu, S. Popovych, S. Mu, W. Wong, C. S. Jordan, M. Castro, J. Buchanan, D. J. Bumbarger, M. Takeno, R. Torres, G. Mahalingam, L. Elabbady, Y. Li, E. Cobos, P. Zhou, S. Suckow, L. Becker, L. Paninski, F. Polleux, J. Reimer, A. S. Tolias, R. C. Reid, N. M. da Costa, H. S. Seung, Multiscale and multimodal reconstruction of cortical structure and function. bioRxiv, 2020.2010.2014.338681 (2020).

76. C. Bas-Orth, Y. W. Tan, D. Lau, H. Bading, Synaptic Activity Drives a Genomic Program That Promotes a Neuronal Warburg Effect. J Biol Chem 292, 5183-5194 (2017).

77. C. M. Diaz-Garcia, R. Mongeon, C. Lahmann, D. Koveal, H. Zucker, G. Yellen, Neuronal Stimulation Triggers Neuronal Glycolysis and Not Lactate Uptake. Cell Metab 26, 361-374 e364 (2017).

78. J. Yoshino, J. A. Baur, S. I. Imai, NAD(+) Intermediates: The Biology and Therapeutic Potential of NMN and NR. Cell Metab 27, 513-528 (2018). 
79. J. Gilley, R. Adalbert, G. Yu, M. P. Coleman, Rescue of peripheral and CNS axon defects in mice lacking NMNAT2. J Neurosci 33, 13410-13424 (2013).

80. H. Currinn, B. Guscott, Z. Balklava, A. Rothnie, T. Wassmer, APP controls the formation of $\mathrm{PI}(3,5) \mathrm{P}(2)$ vesicles through its binding of the PIKfyve complex. Cell Mol Life Sci 73, 393-408 (2016).

81. M. R. Galiano, S. Jha, T. S. Ho, C. Zhang, Y. Ogawa, K. J. Chang, M. C. Stankewich, P. J. Mohler, M. N. Rasband, A distal axonal cytoskeleton forms an intra-axonal boundary that controls axon initial segment assembly. Cell 149, 1125-1139 (2012).

82. B. M. Kitay, R. McCormack, Y. Wang, P. Tsoulfas, R. G. Zhai, Mislocalization of neuronal mitochondria reveals regulation of Wallerian degeneration and NMNAT/WLD(S)-mediated axon protection independent of axonal mitochondria. Hum Mol Genet 22, 1601-1614 (2013).

83. T. L. Fletcher, P. Cameron, P. De Camilli, G. Banker, The distribution of synapsin I and synaptophysin in hippocampal neurons developing in culture. J Neurosci 11, 1617-1626 (1991). 
Fig. 1

A NMNAT2 ${ }^{f}$

Exon1 LTR-DWW NMNAT2 cKO $\downarrow$ Nex-Cre

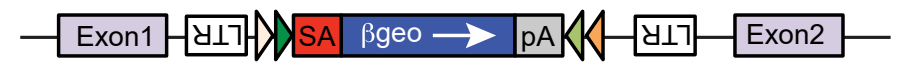

B

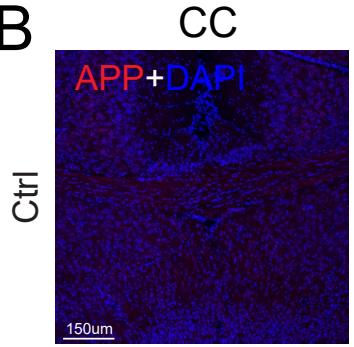

$\stackrel{\bigcirc}{\mathrm{y}}$

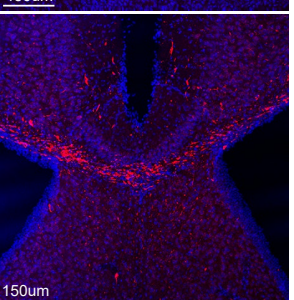

Hipp
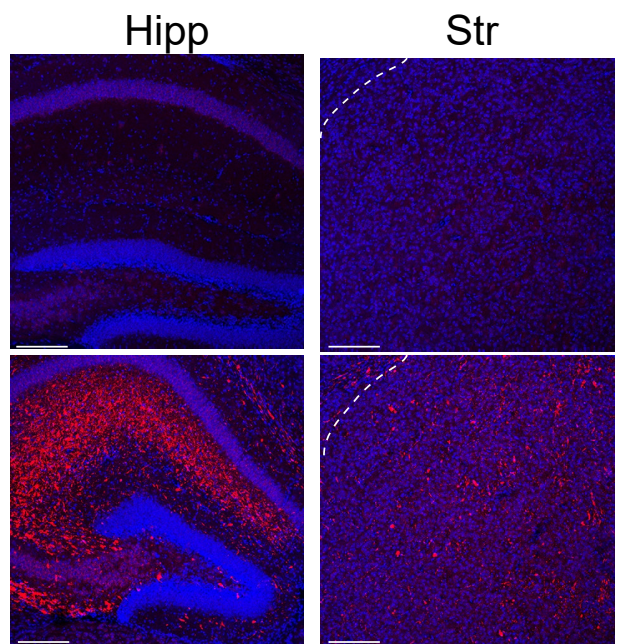

C

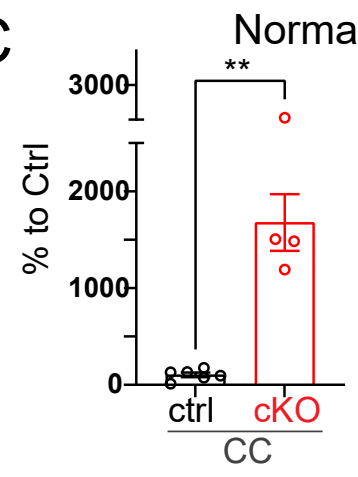

Normalized APP pixel values

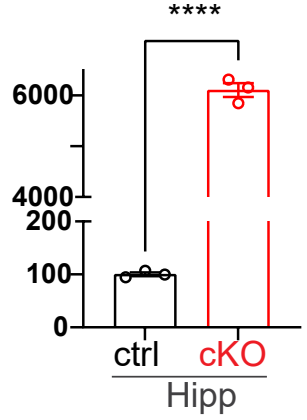

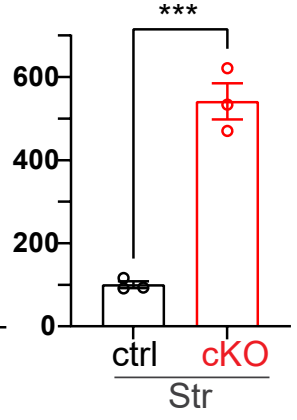

D

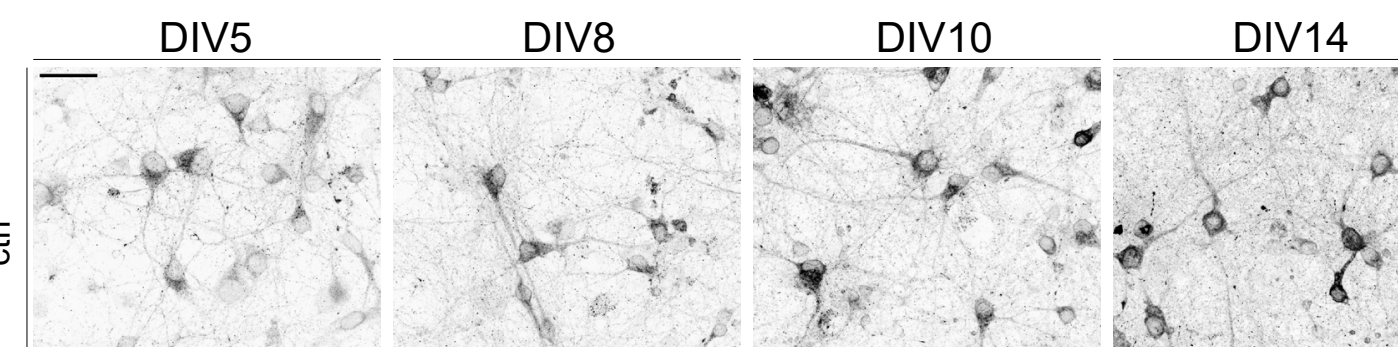

APP

$\underset{\vartheta}{\gtrless}$

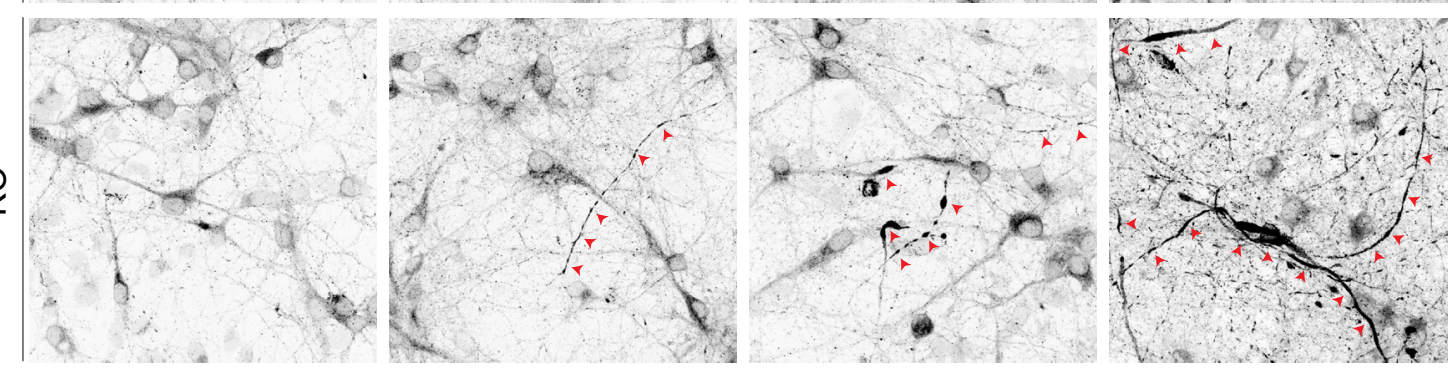

$E$
F

Normalized APP

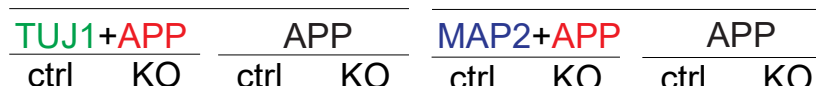

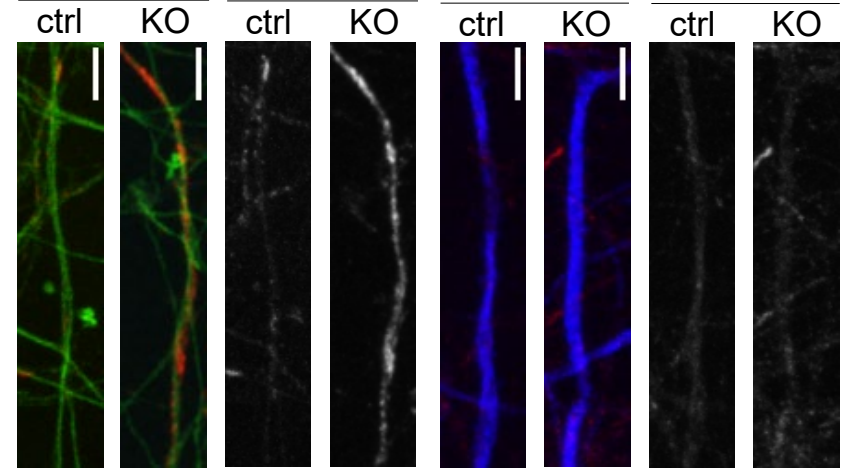

accumulation in axons

${ }^{500} \mathrm{ctrl}$

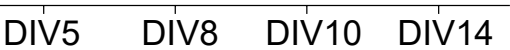

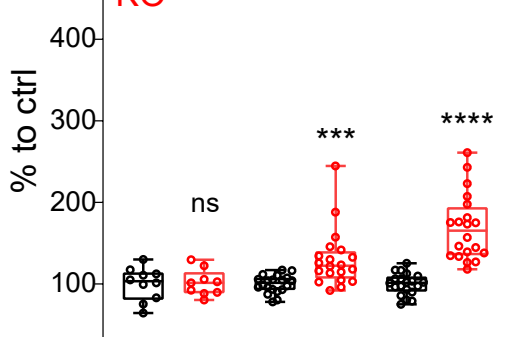


Fig. 1-S1

A

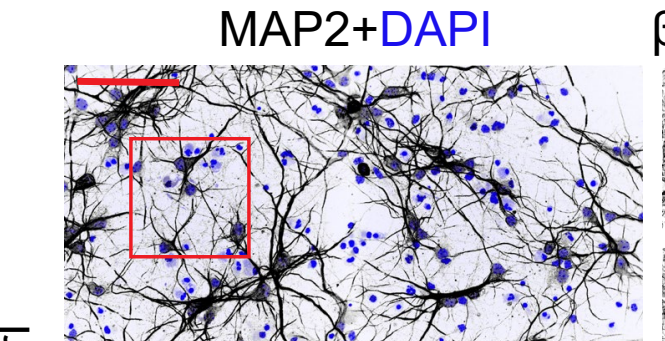

BIII-Tubulin (subtract MAP2)

MAP2+TUJ1+DAPI

志

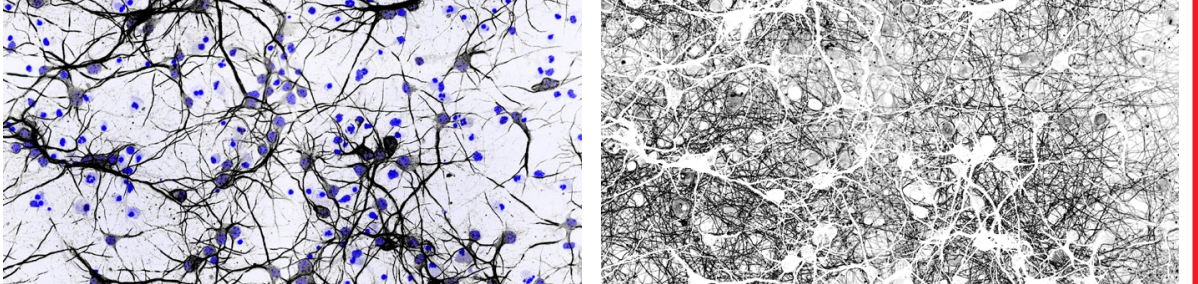

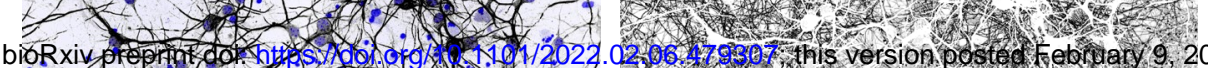

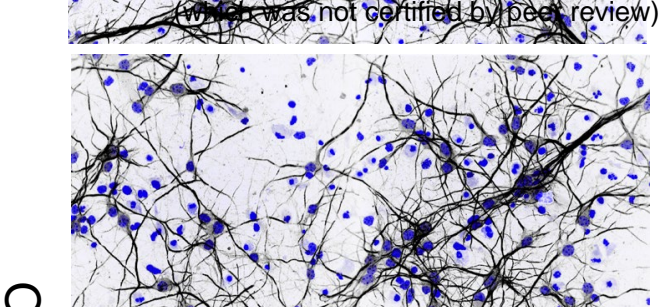

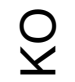
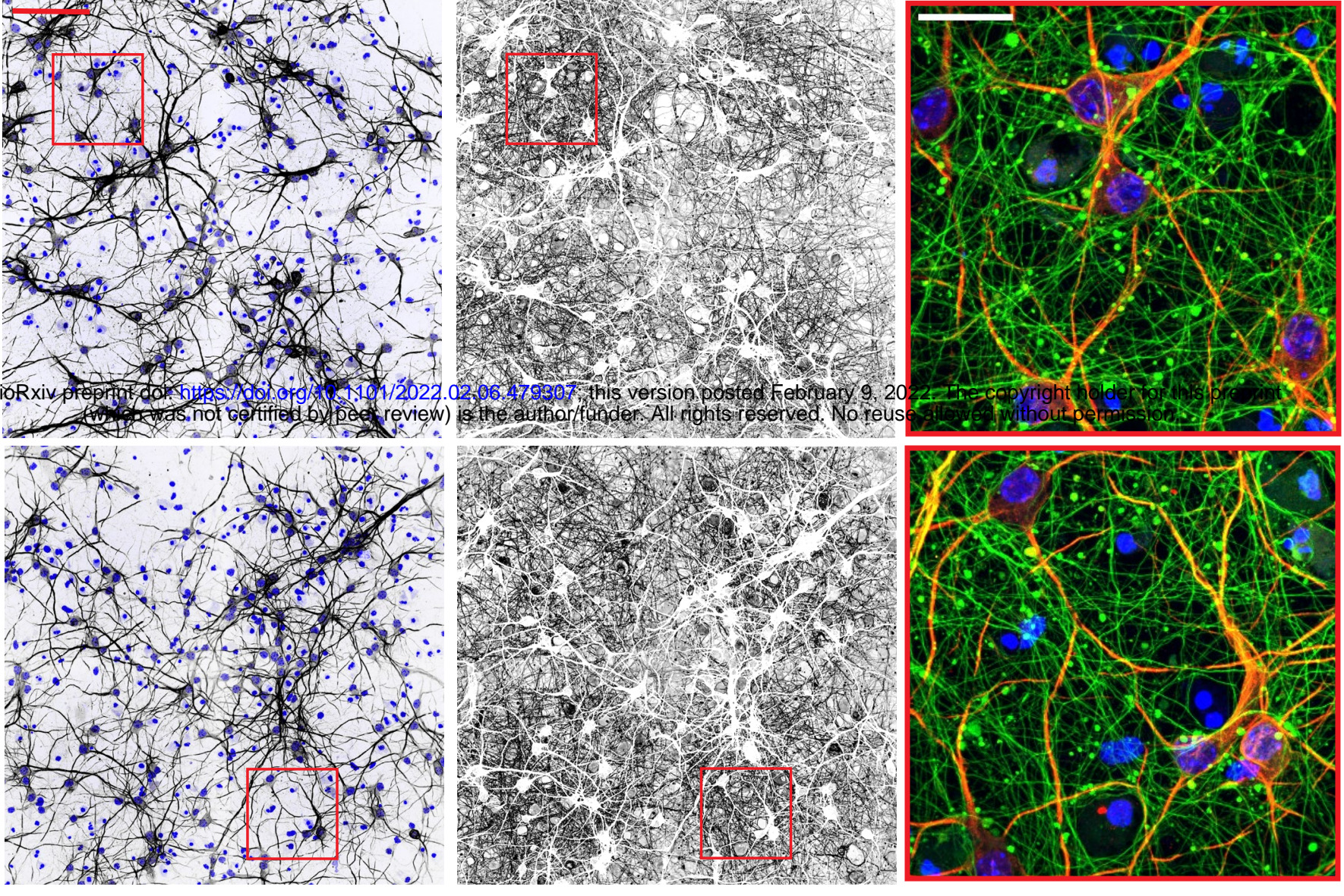

B

Normalized MAP2 area

Normalized TUJ1 area
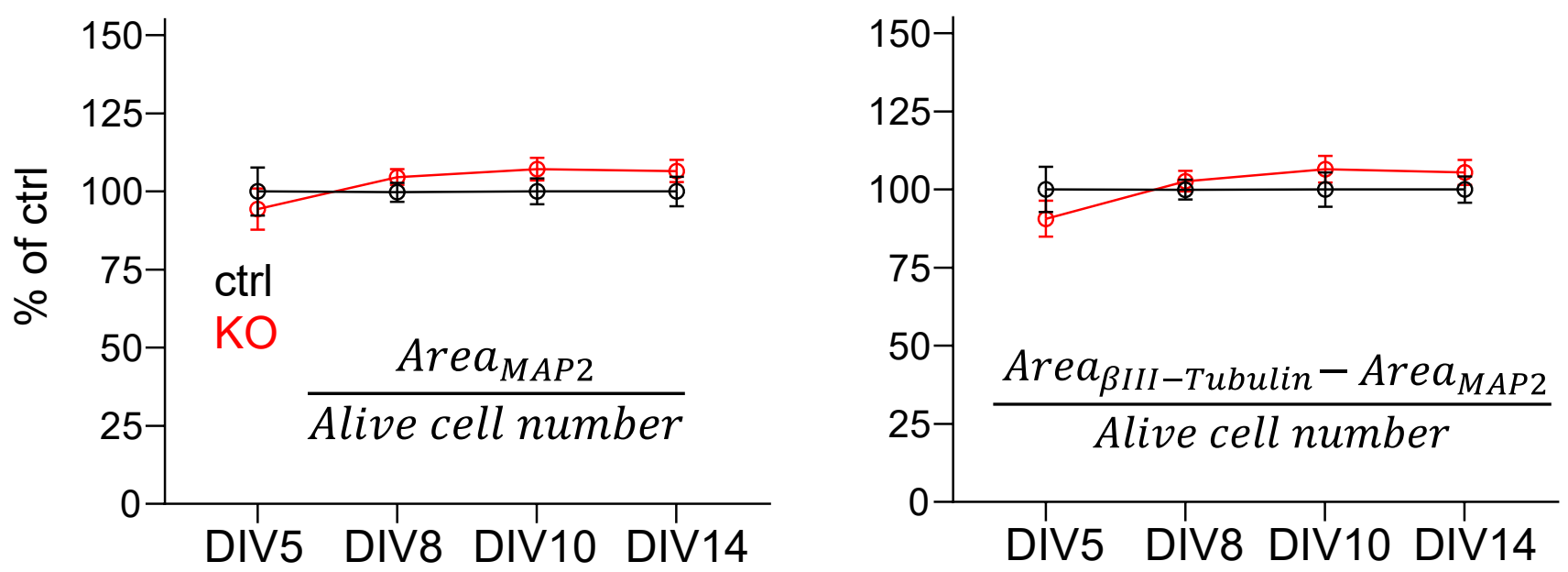

$\mathrm{C}$

quantification method for APP accumulations in neurites

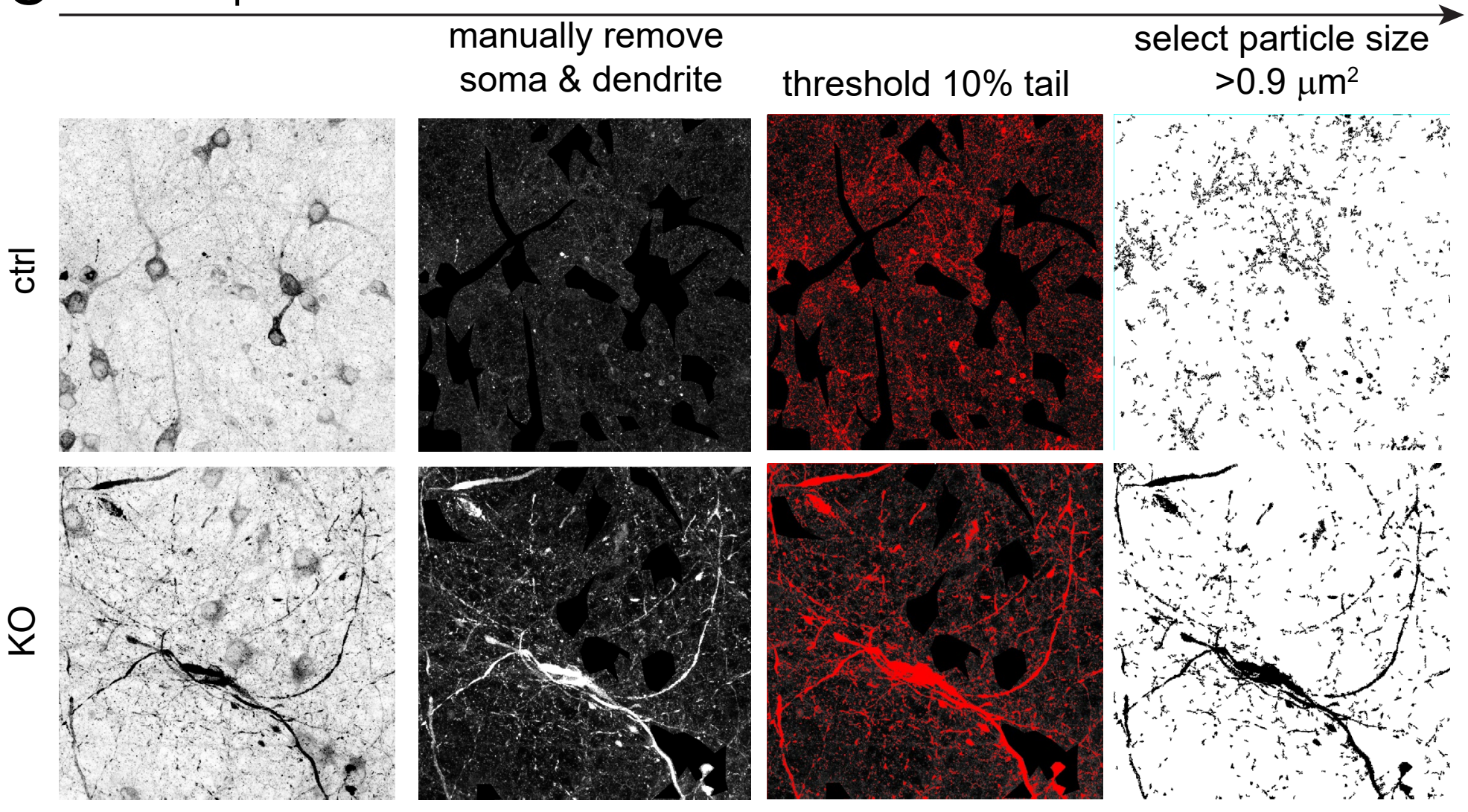


Fig. 1-S2
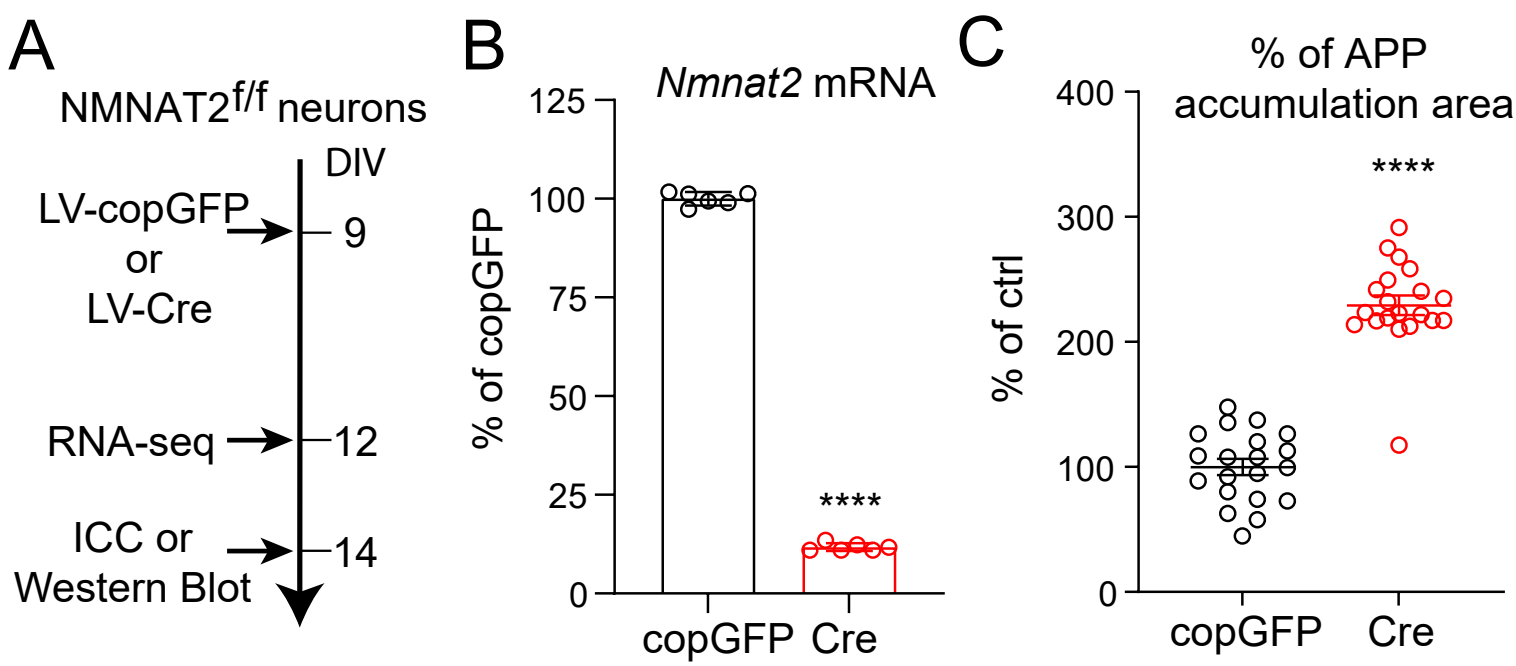

D

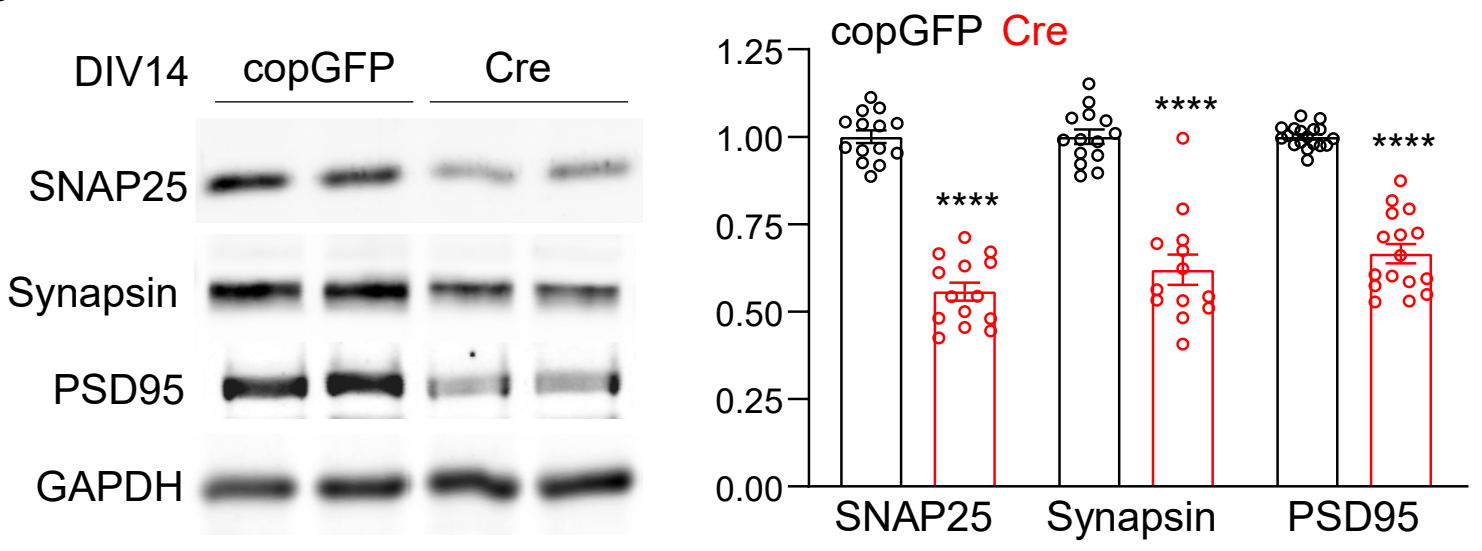

fold change to ctrl 
Fig. 2
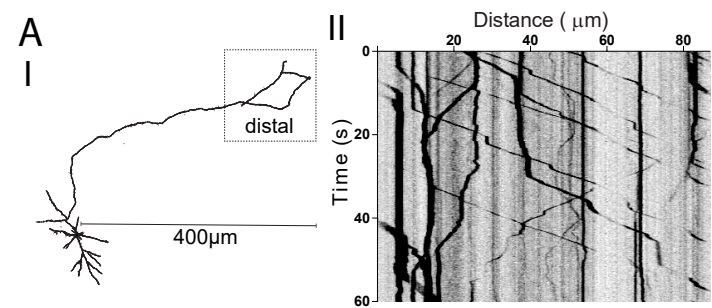

$\square$ Anterograde $\square$ Retrograde $\square$ Stationary/Dynamic Pause
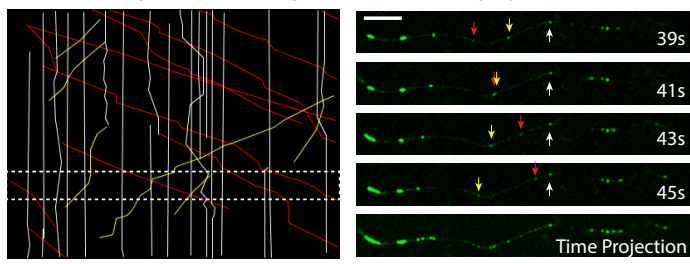

B

APP

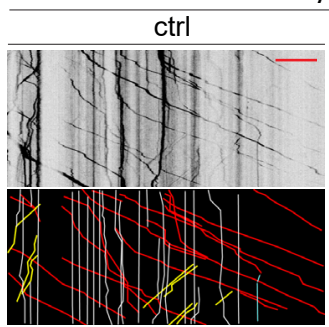

E

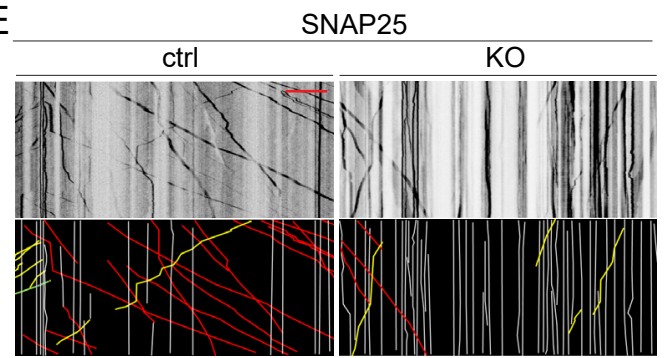

C

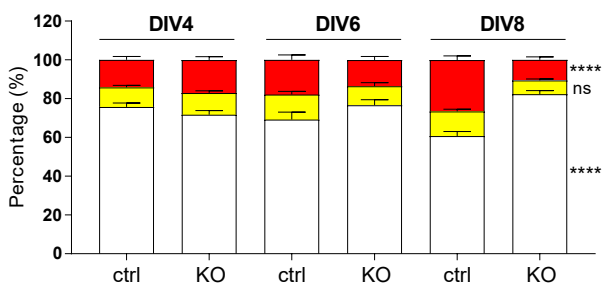

$\mathrm{F}$

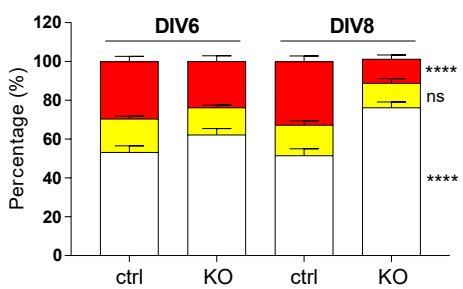

D Anterograde Velocity Retrograde Velocity
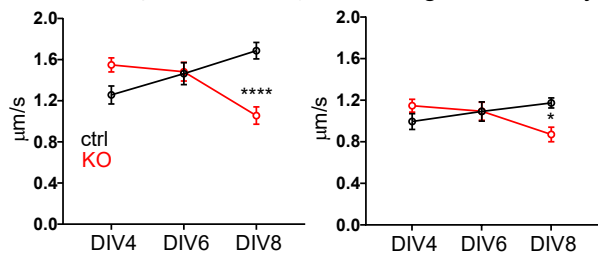

G Anterograde Velocity
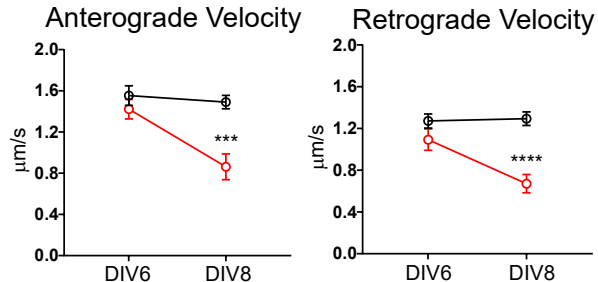
Fig. 2-S1
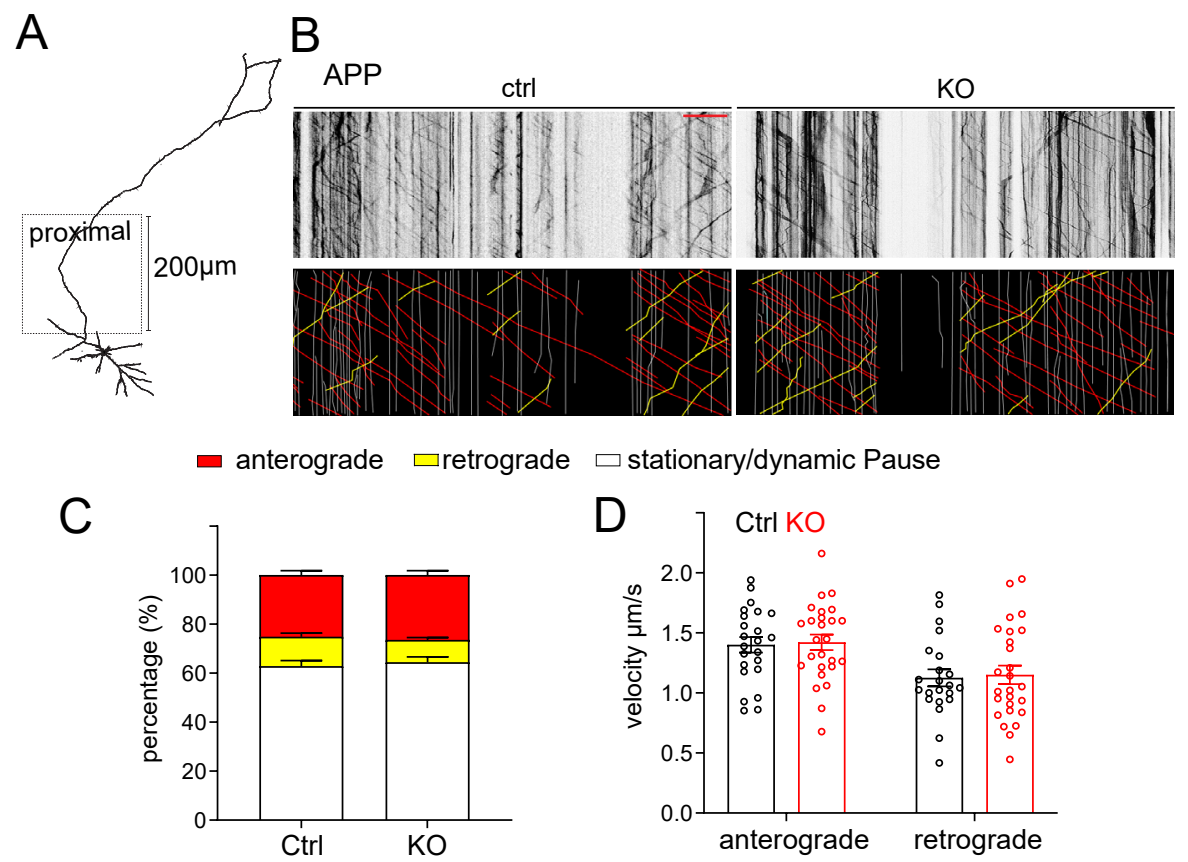

- anterograde $\square$ retrograde $\square$ stationary/dynamic Pause

E SNAP25
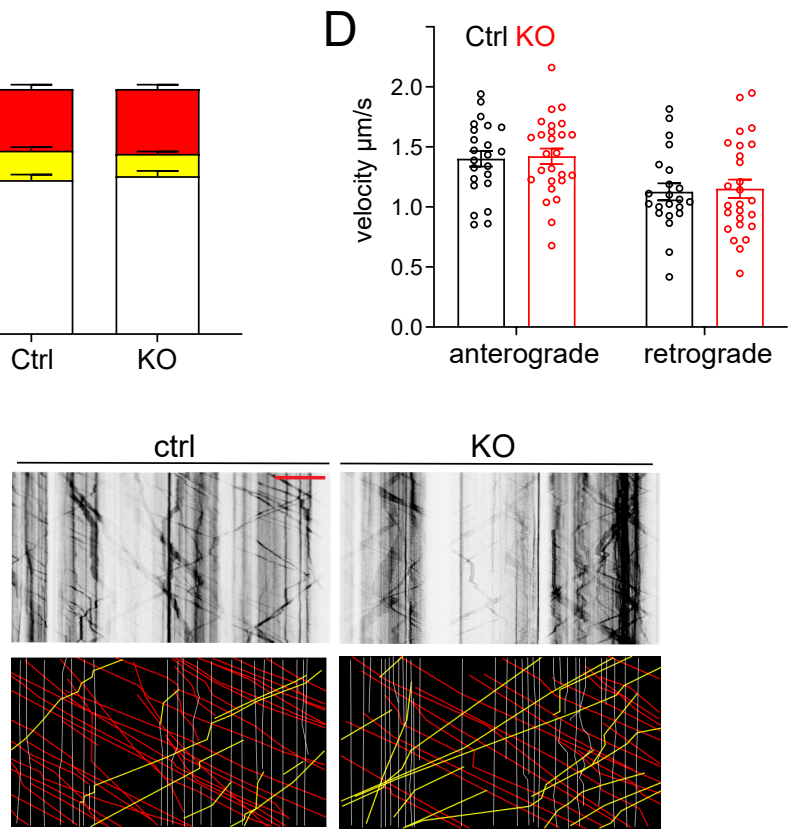

$\mathrm{F}$

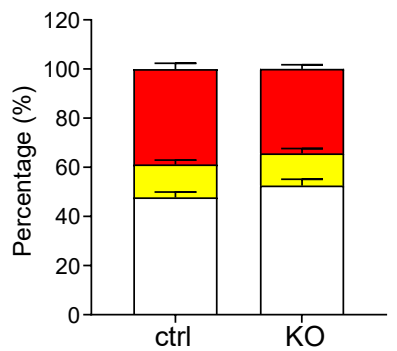

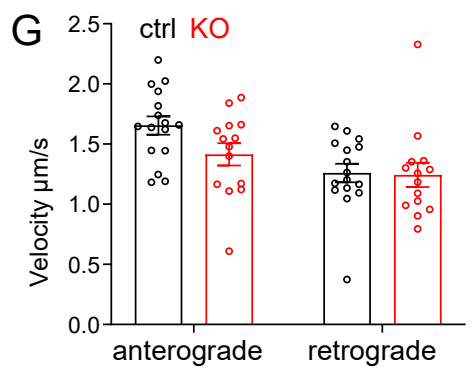




\section{Fig. 2-S2}

A

ctrl

KO

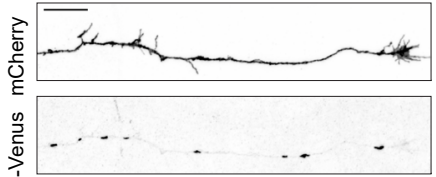

B Mitochondria Density C
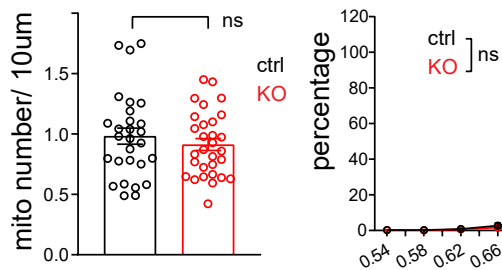

D

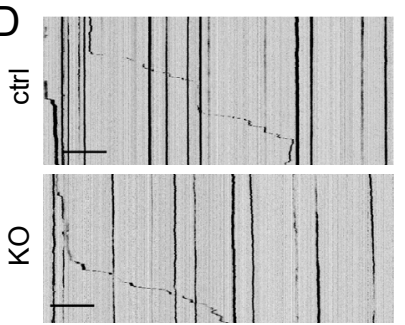

E Anterograde

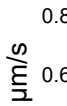

$\begin{array}{ll}\frac{\lambda}{\mathrm{O}} & 0.4- \\ \frac{\mathrm{O}}{\mathrm{J}} & 0.2\end{array}$ 0.0

Anterograde

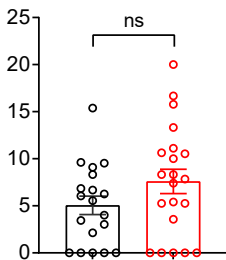

Retrograde

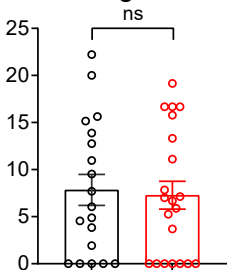

Stationary

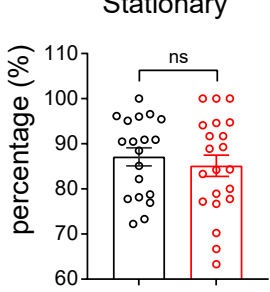

Mitochondria Morphology

Retrograde

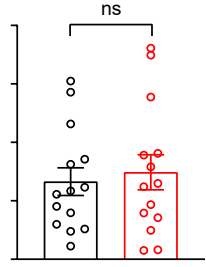

$\mathrm{F}$ 
Fig. 3
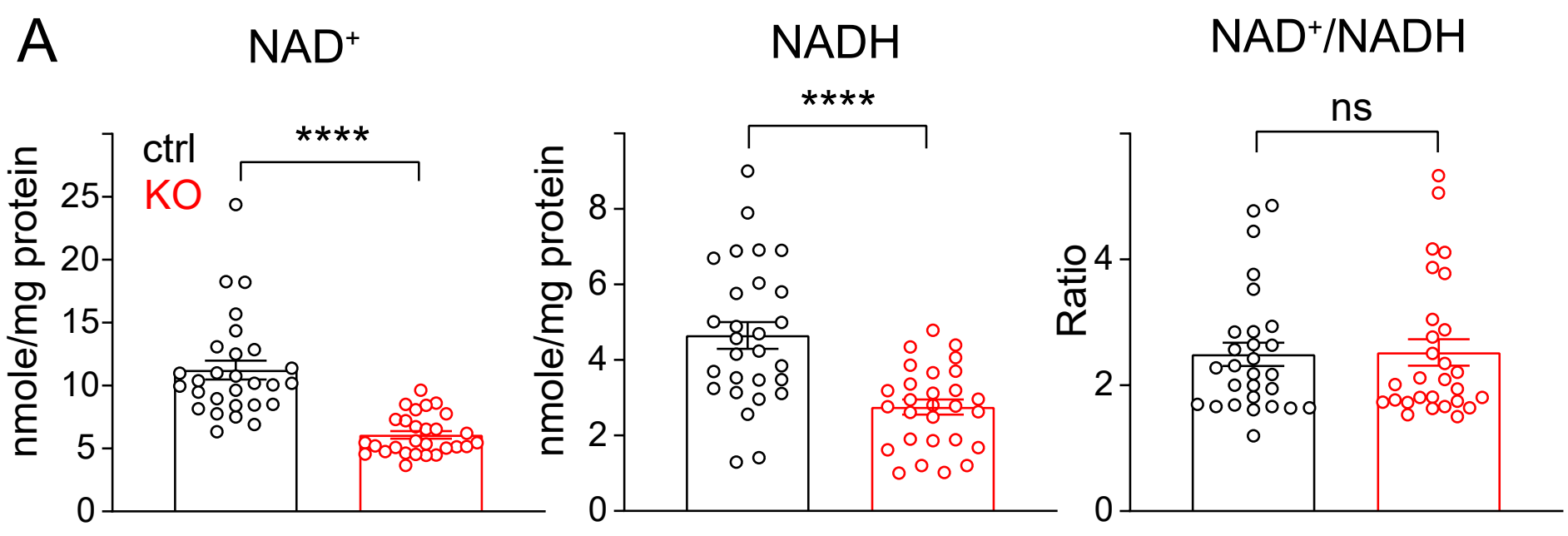

B
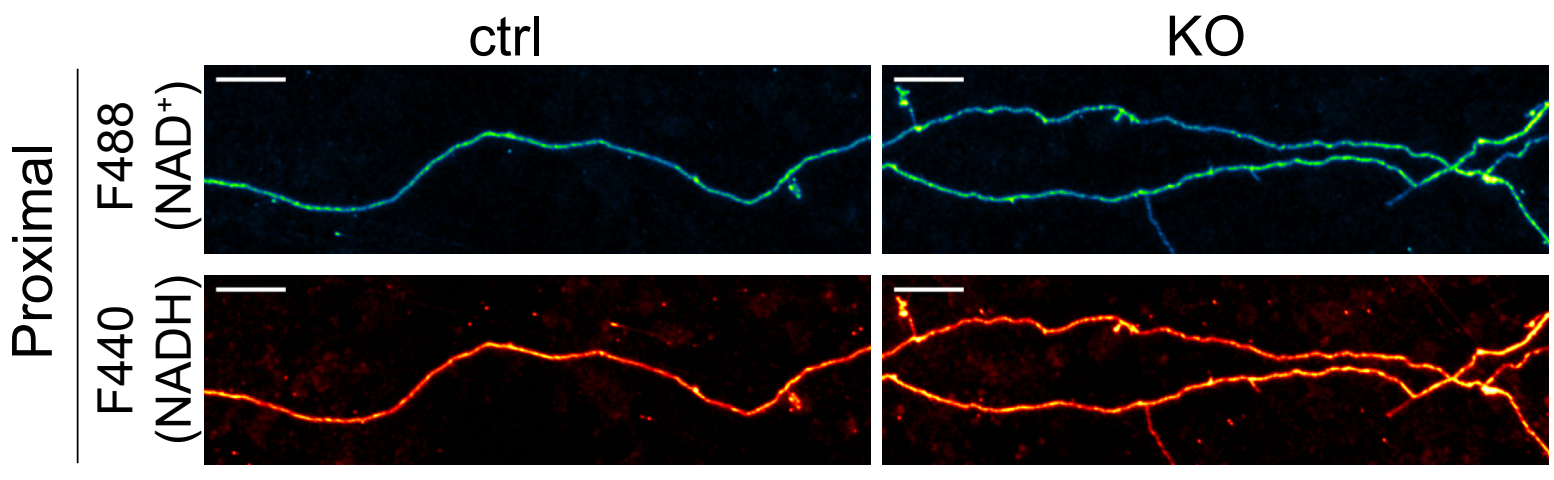

F488
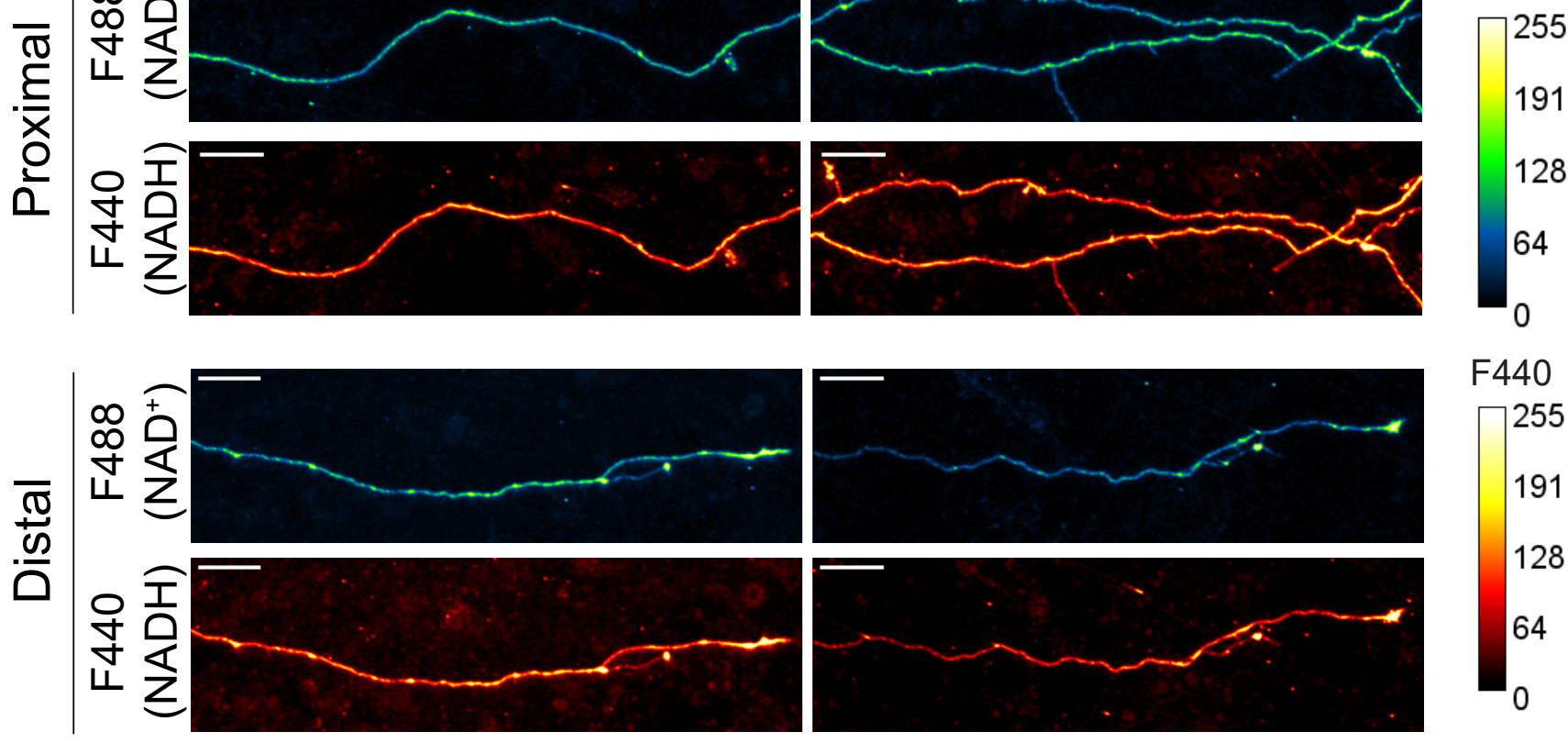

F440

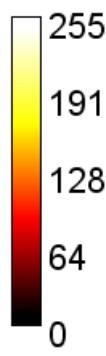

$\mathrm{NAD}^{+} / \mathrm{NADH}$ (SoNar)

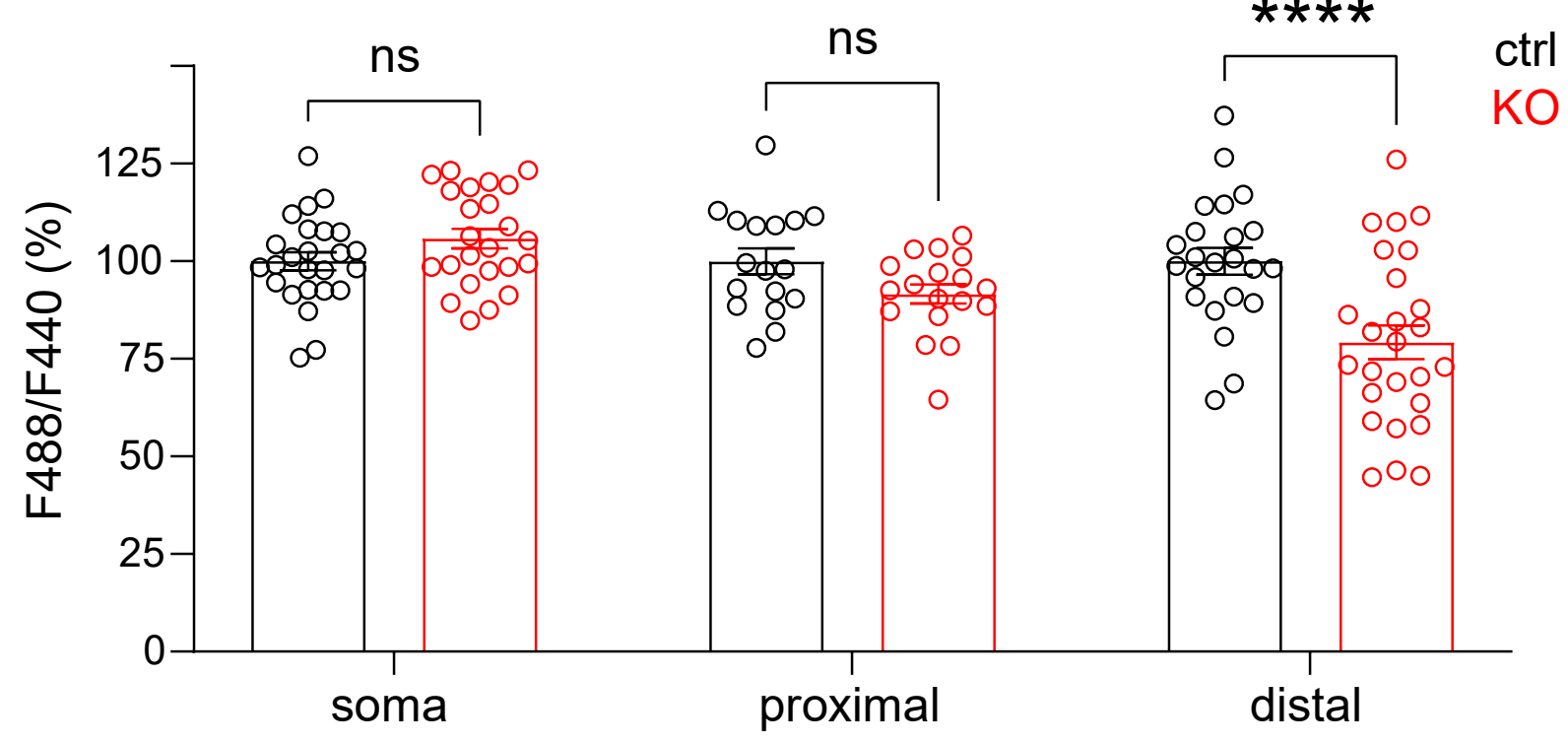


Fig. 4

A

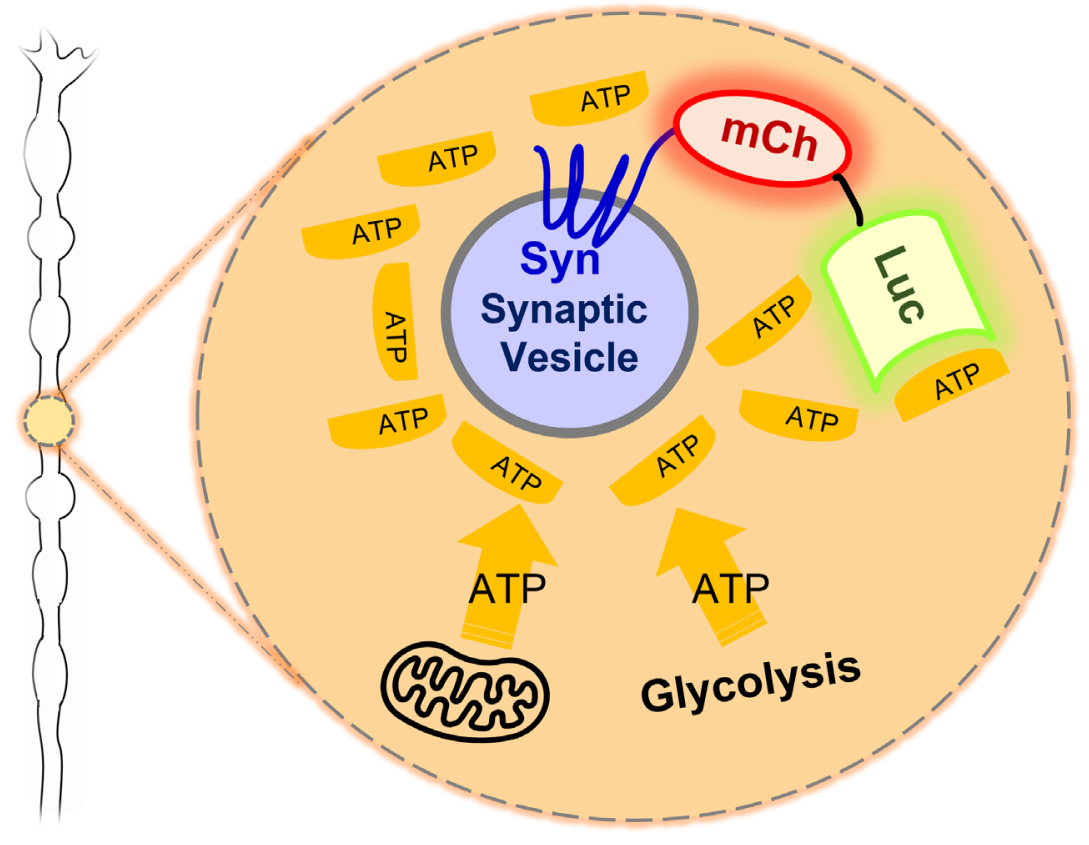

Timeline $\underset{\text { imaging }}{\stackrel{\text { basal }}{\stackrel{\text { bmin }}{+ \text { oligomycin }}<=30 \mathrm{~min}}}$

C

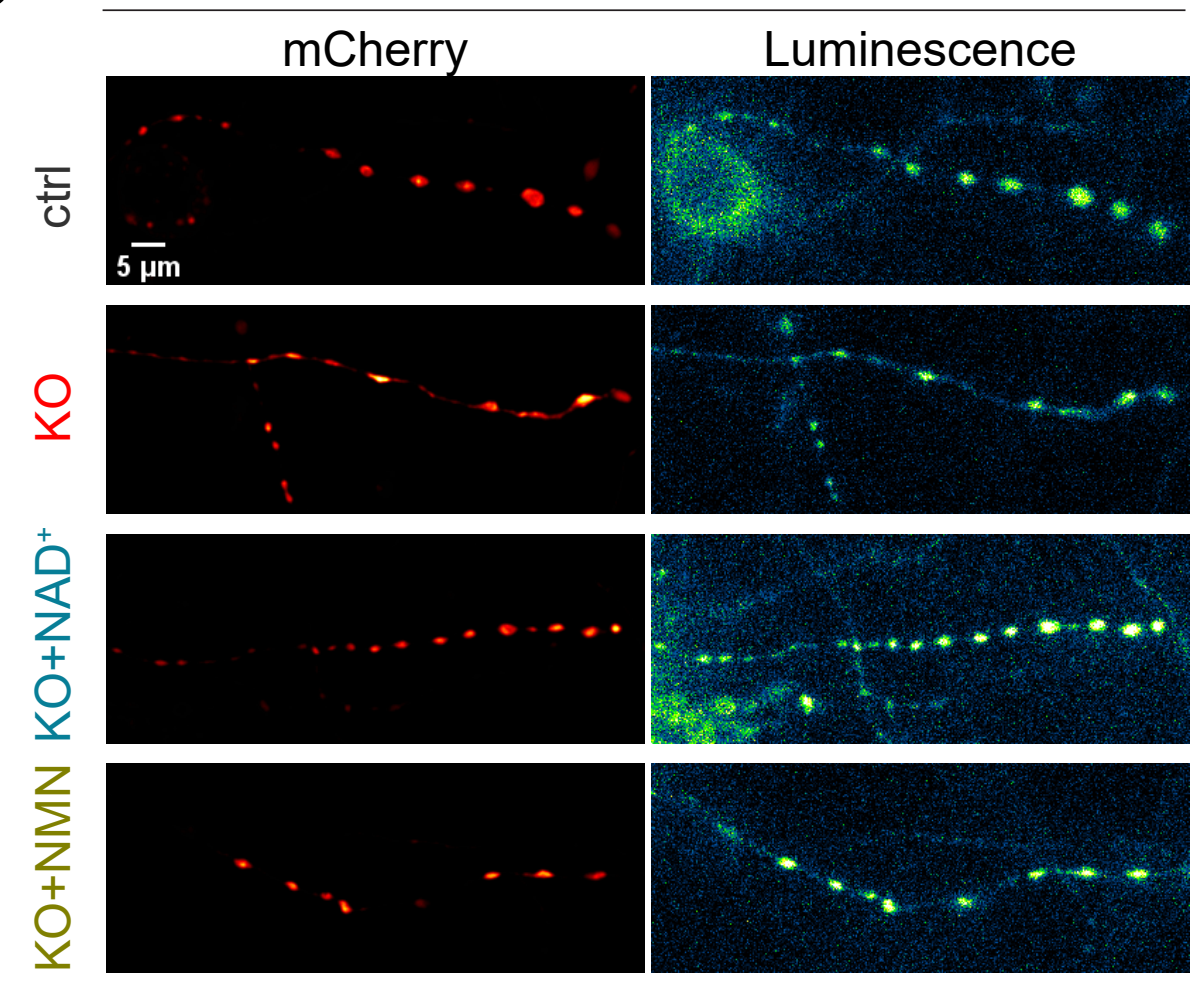

B

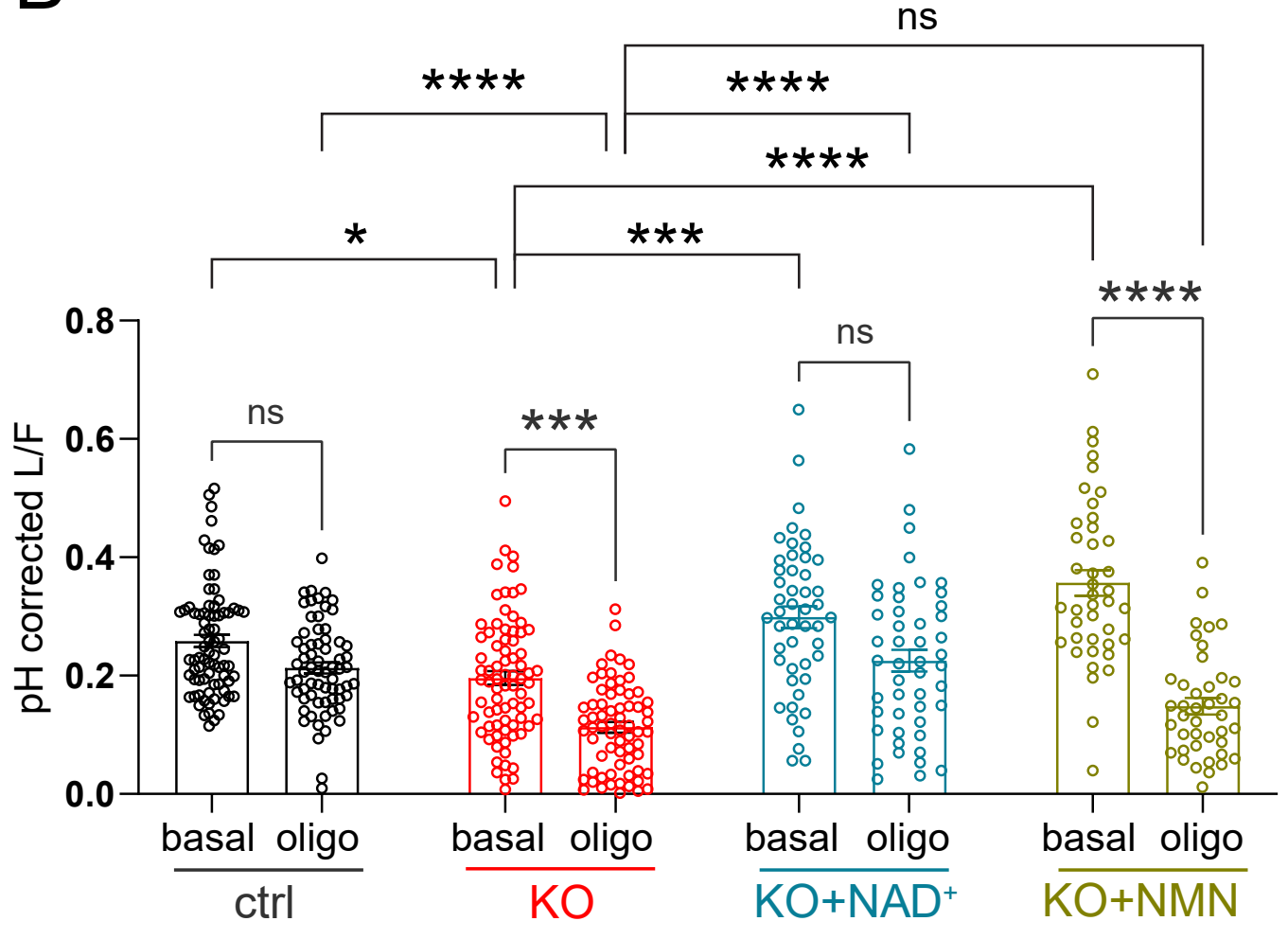

Oligomycin

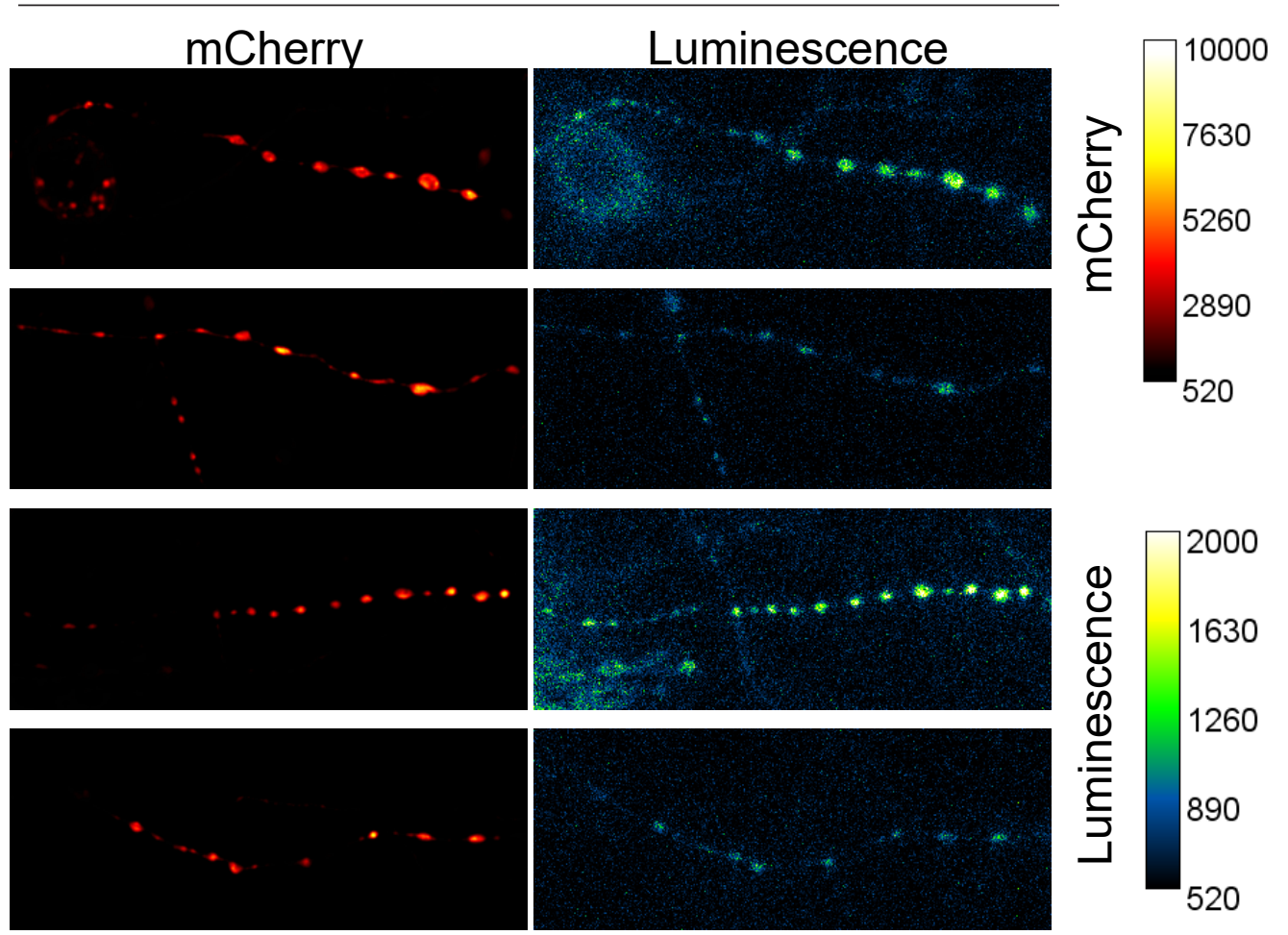


A CytopHluorin imaging timeline

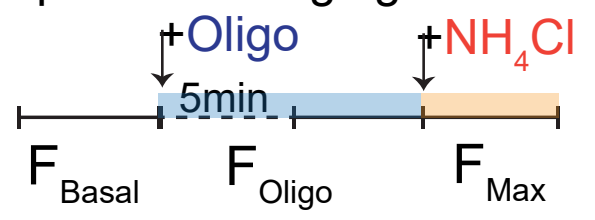

$\mathrm{pH}$ calculation
$p H_{\text {Basal }}=\mathrm{pKa}-\log \left[\left(\frac{1+10^{p K a-7.4}}{\left.\left.\frac{F_{\text {Basal }}}{F_{\text {Max }}}\right)-1\right]}\right.\right.$
$p H_{\text {Oligo }}=\mathrm{pKa}-\log \left[\left(\frac{1+10^{\text {pKa-7.4 }}}{\frac{F_{\text {Oligo }}}{F_{\text {Max }}}}\right)-1\right]$
$p K a=7.1$ for CytopHluorin

C

\begin{tabular}{|c|}
\hline $\mathrm{pH}$ correction of L/F \\
$L_{1} \sim$ Luminescence under $p H_{1}$ \\
$L_{0} \sim$ Luminescence under $p H_{0}$ \\
$p H_{1}=\mathrm{pKa}-\log \left[\left(\frac{1+10^{p K a-p H_{0}}}{\frac{L_{1}}{L_{0}}}\right)-1\right]$ \\
$L_{0}=L_{1} *\left(\frac{1+10^{p K a-p H_{1}}}{1+10^{p K a-p H_{0}}}\right)$ \\
$p K a=7.03$ for SynATP \\
eg. Convert L in KO+NMN basal group (average pH=6.863) \\
to ctrl basal condition (average pH=7.076) \\
$L_{(p H=7.076)}=L_{(p H=6.863)} *\left(\frac{1+10^{7.03-6.863}}{1+10^{7.03-7.076}}\right)$
\end{tabular}

B

$\mathrm{pH}$ measurement

ctrl $\mathrm{KO} \mathrm{KO}+\mathrm{NAD}^{+} \mathrm{KO}+\mathrm{NMN}$
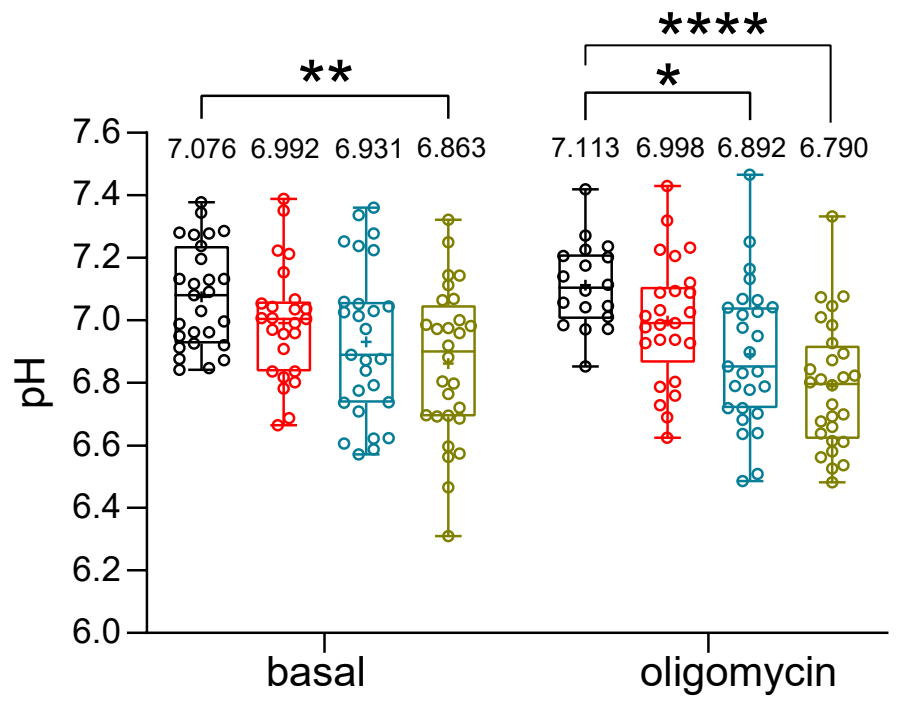

D

\section{Syn-ATP measurement in ctrl}

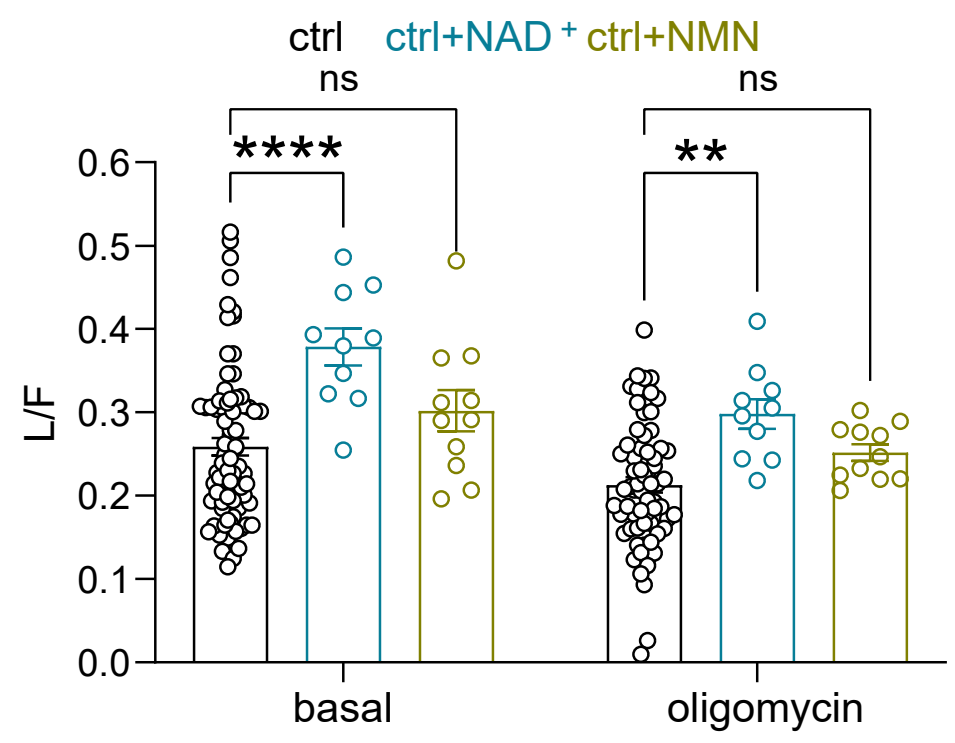


Fig. 5
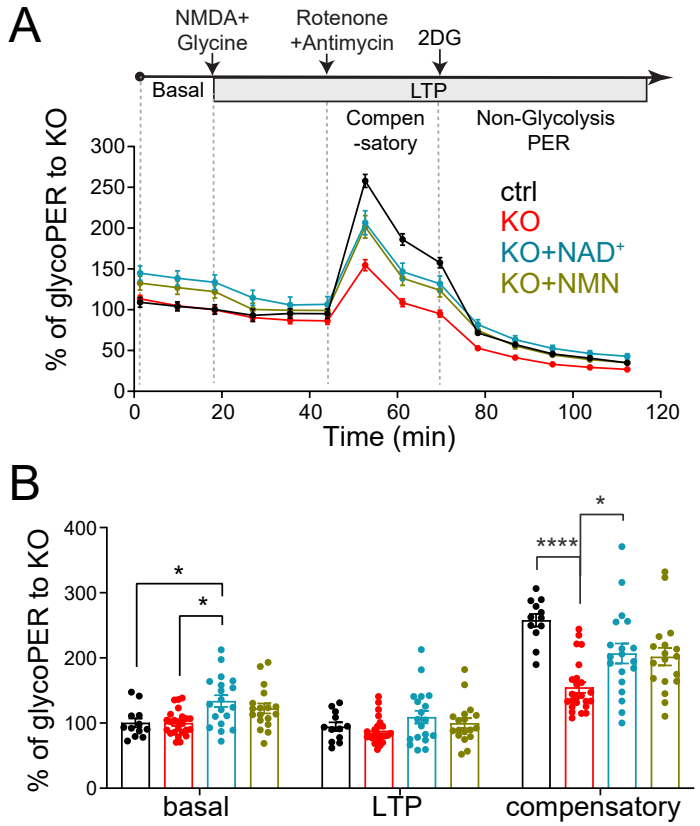
Fig. 5-S1
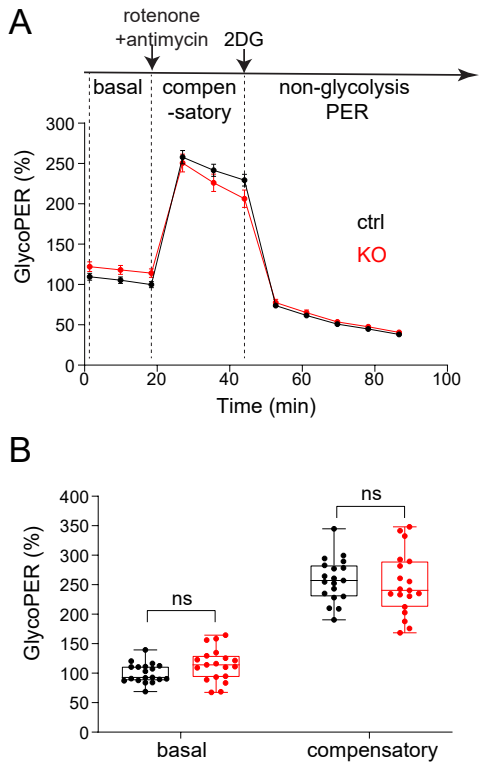
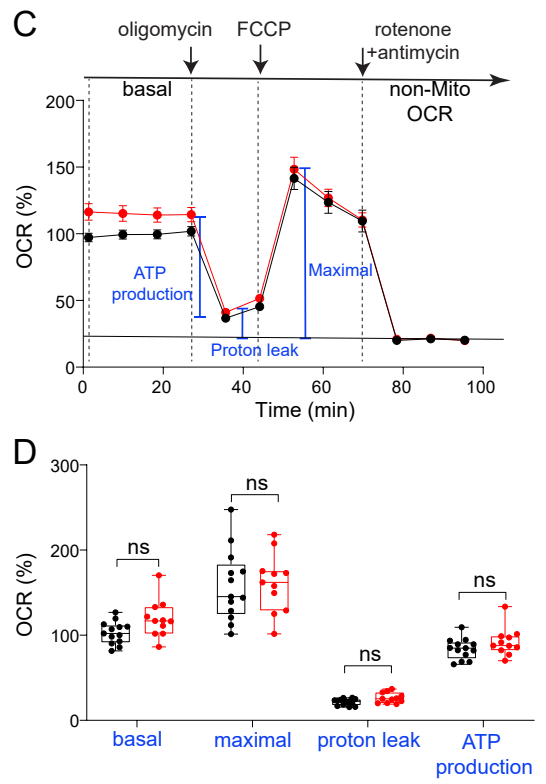
Fig. 6

A
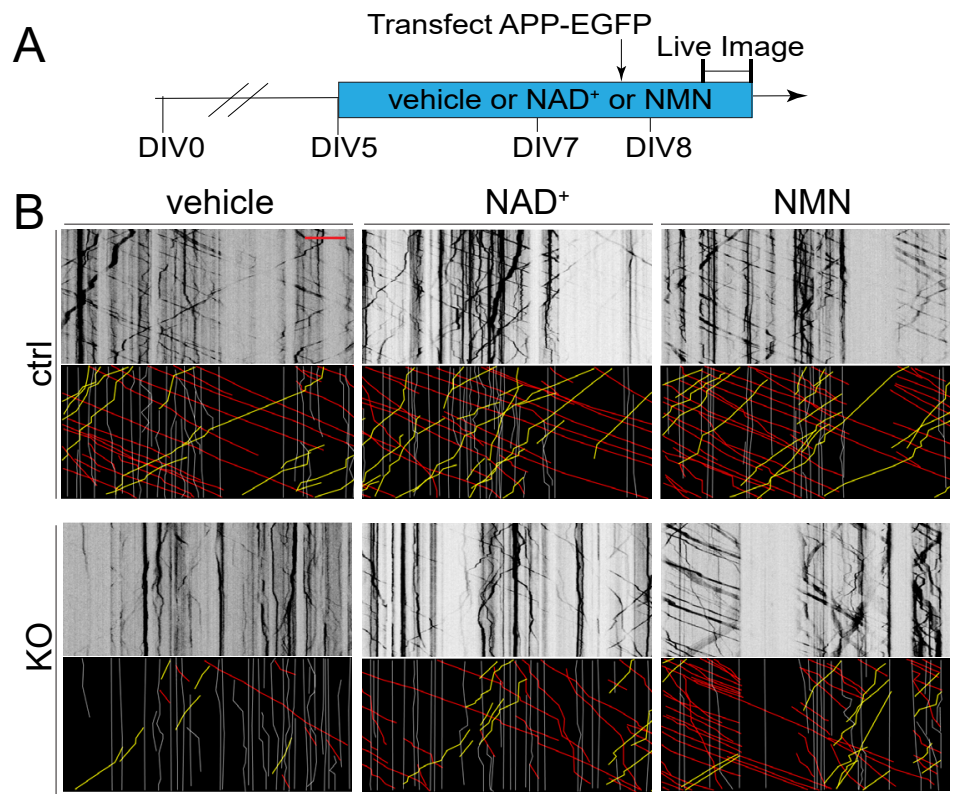

$\square$ Anterograde $\square$ Retrograde $\square$ Stationary/Dynamic Pause

C

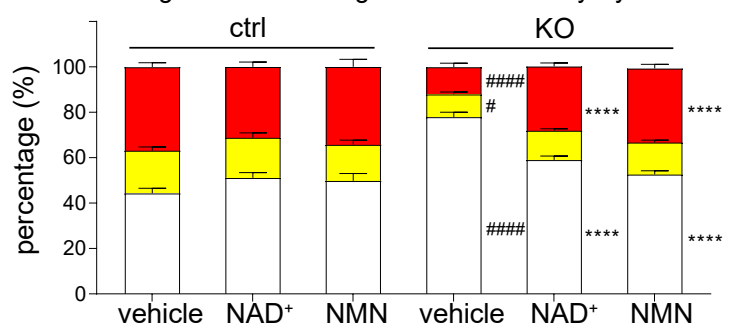

D

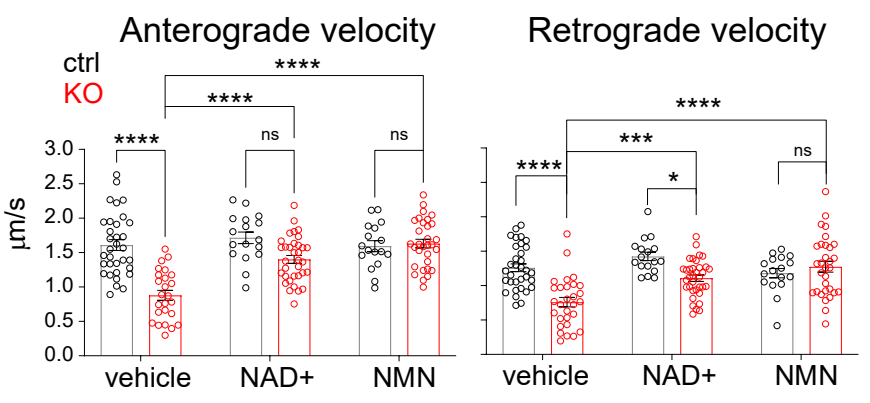


Fig. 7
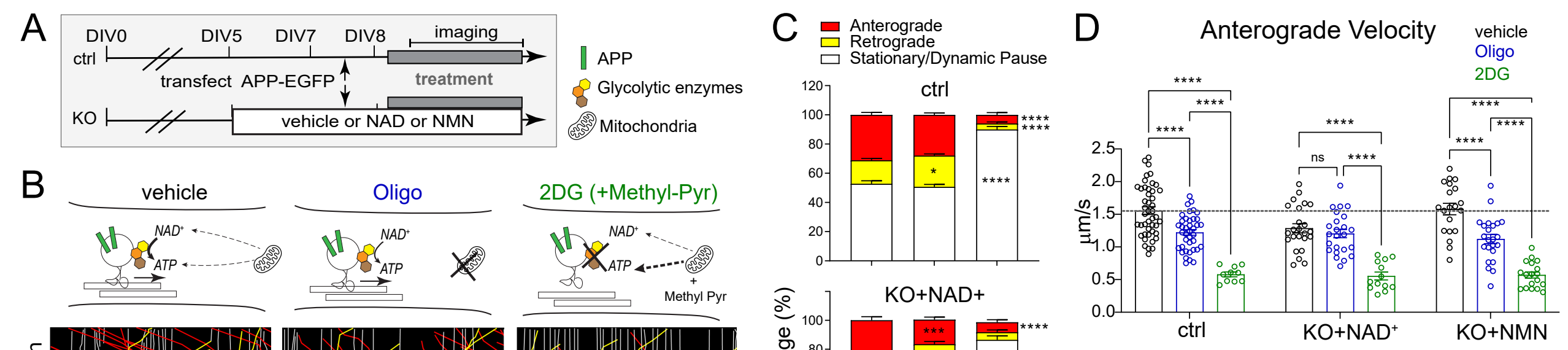

B
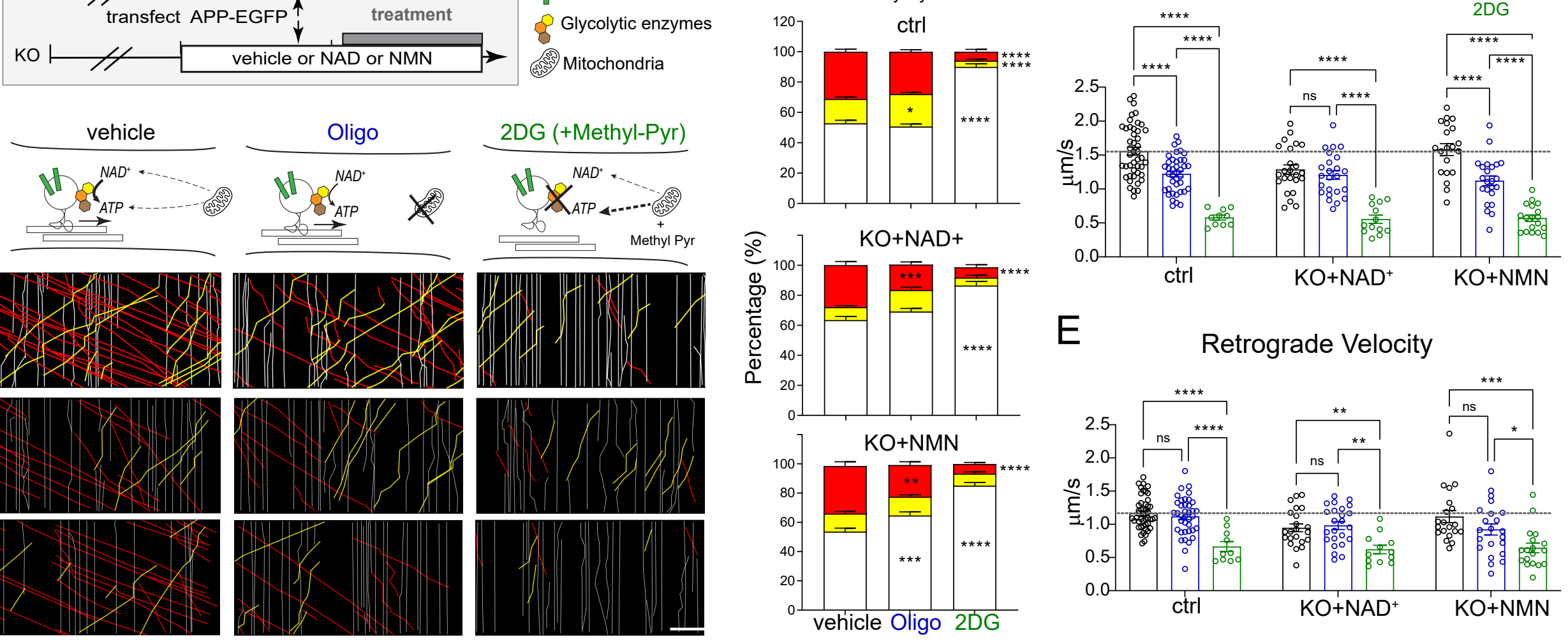

E

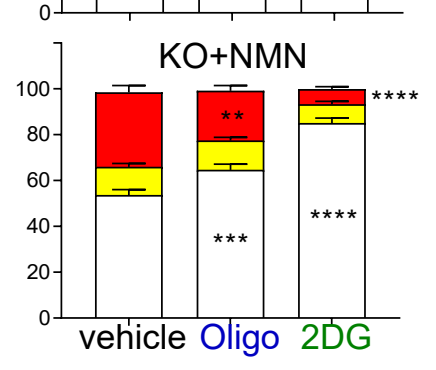

\section{Retrograde Velocity}

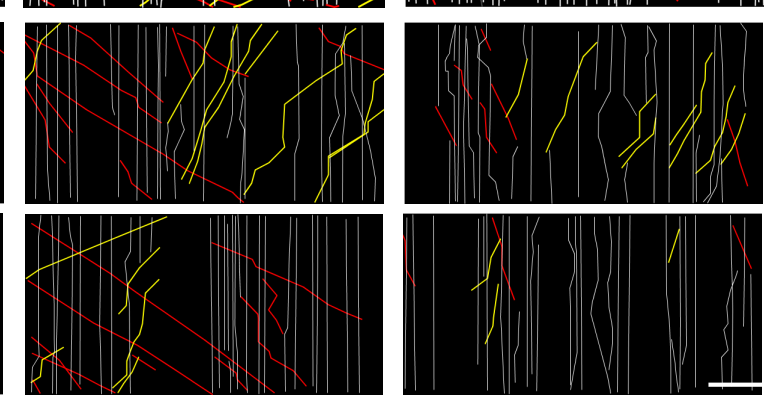


Fig. 8

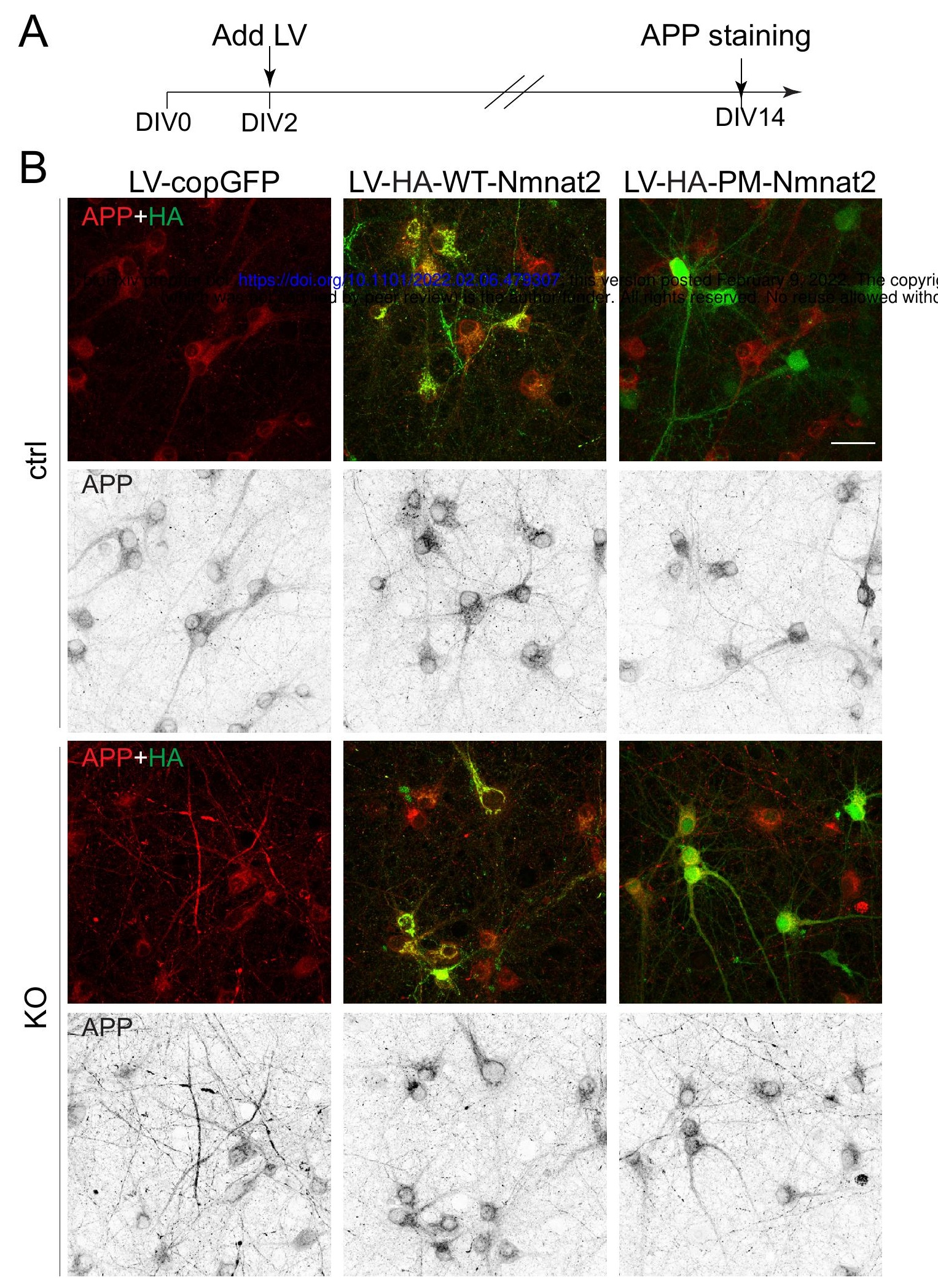

C

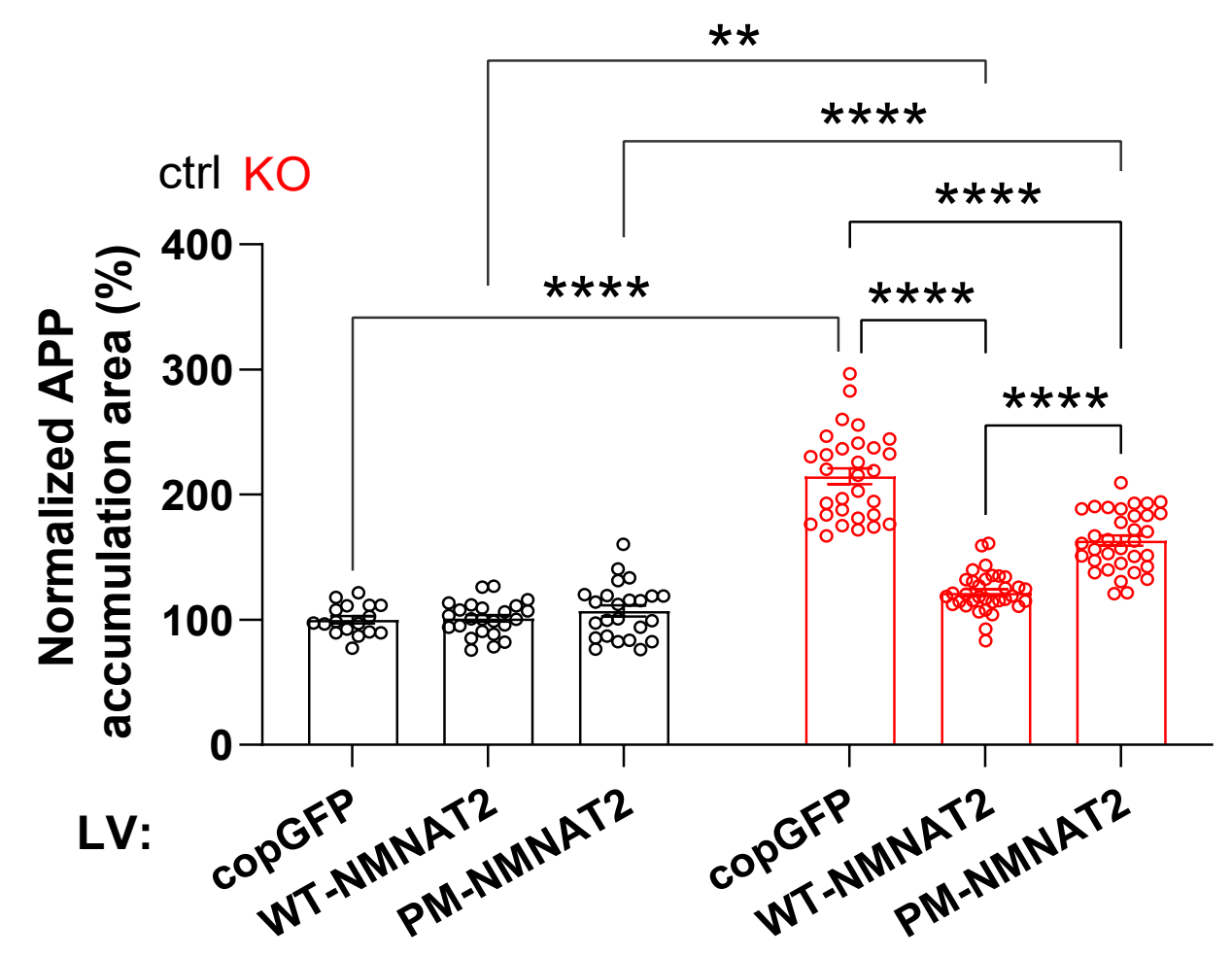


Fig. 8-S1

A

Proximal

Distal
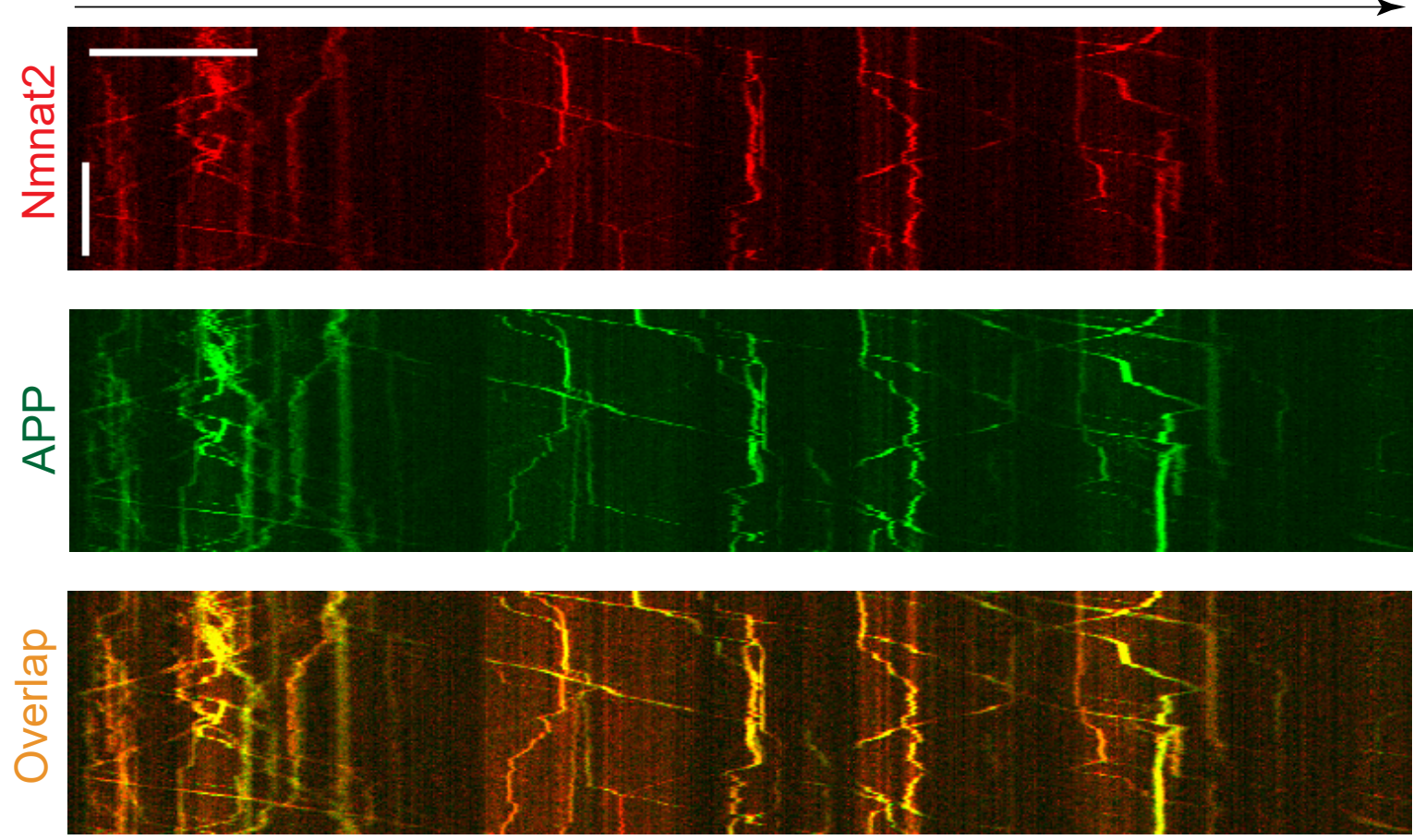

B

Anterograde Velocity

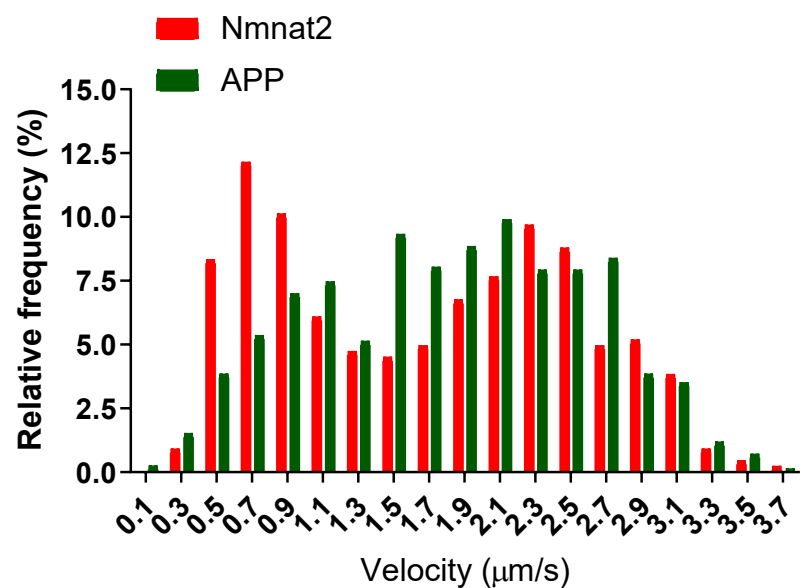

Retrograde Velocity

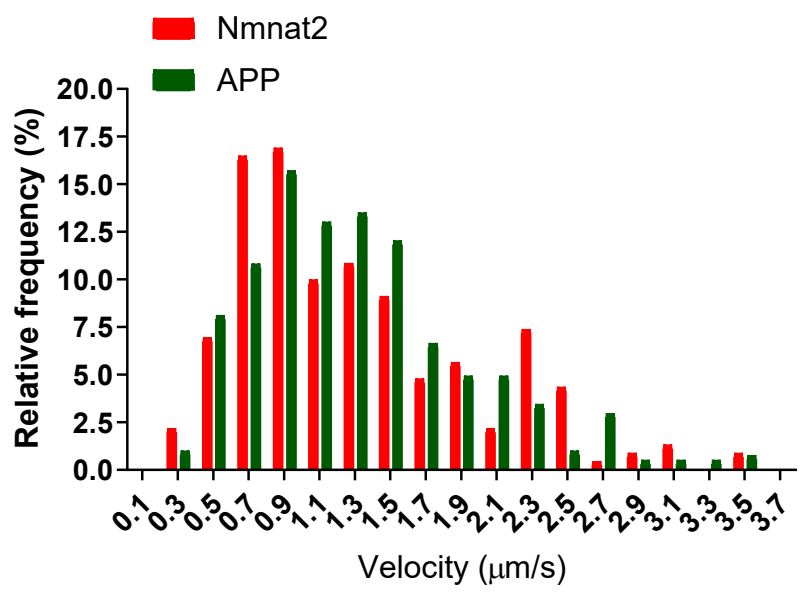


Fig. 8-S2

A LV-copGFP:

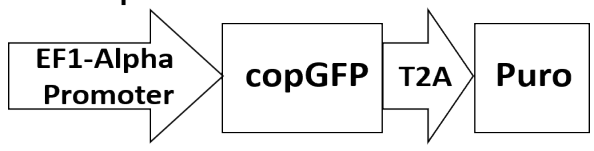

LV-HA-Nmnat2:

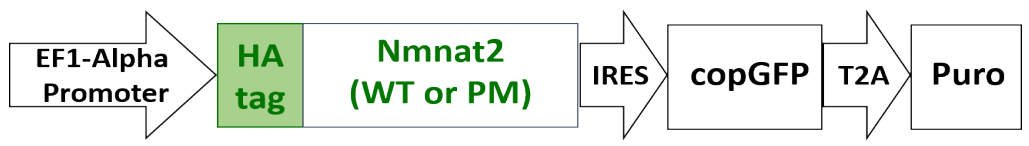

B

Lentiviral transduction rate $=\frac{\# A P P \text { positive cells with } H A \text { expression }}{\# A P P \text { positive cells }} * 100 \%$ $3 \mathrm{MOI} \mathrm{LV}$ to $\mathrm{KO}$ neurons

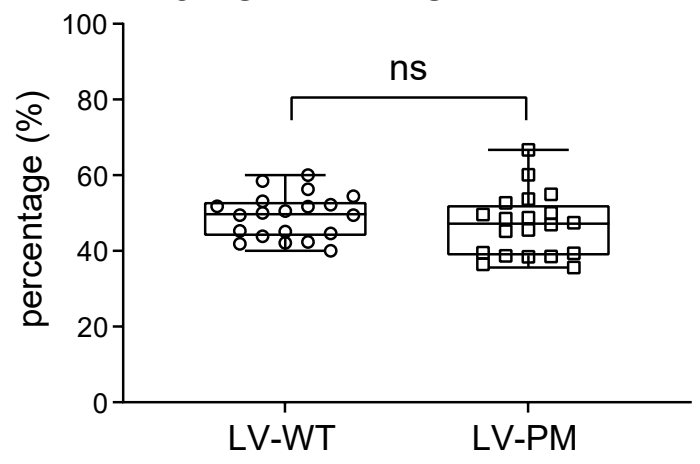

$8 \mathrm{MOI} \mathrm{LV}$ to $\mathrm{KO}$ neurons

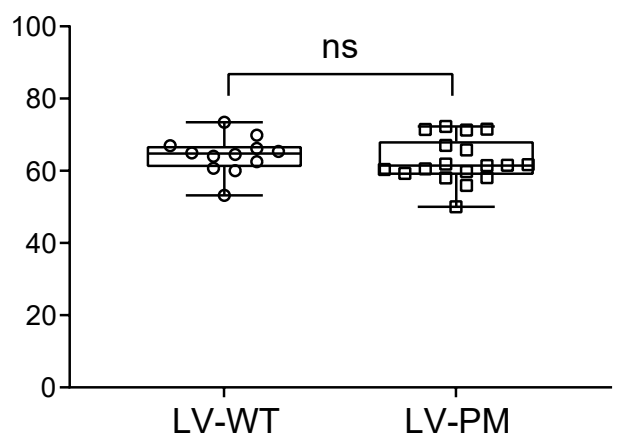

C

2MOI LV
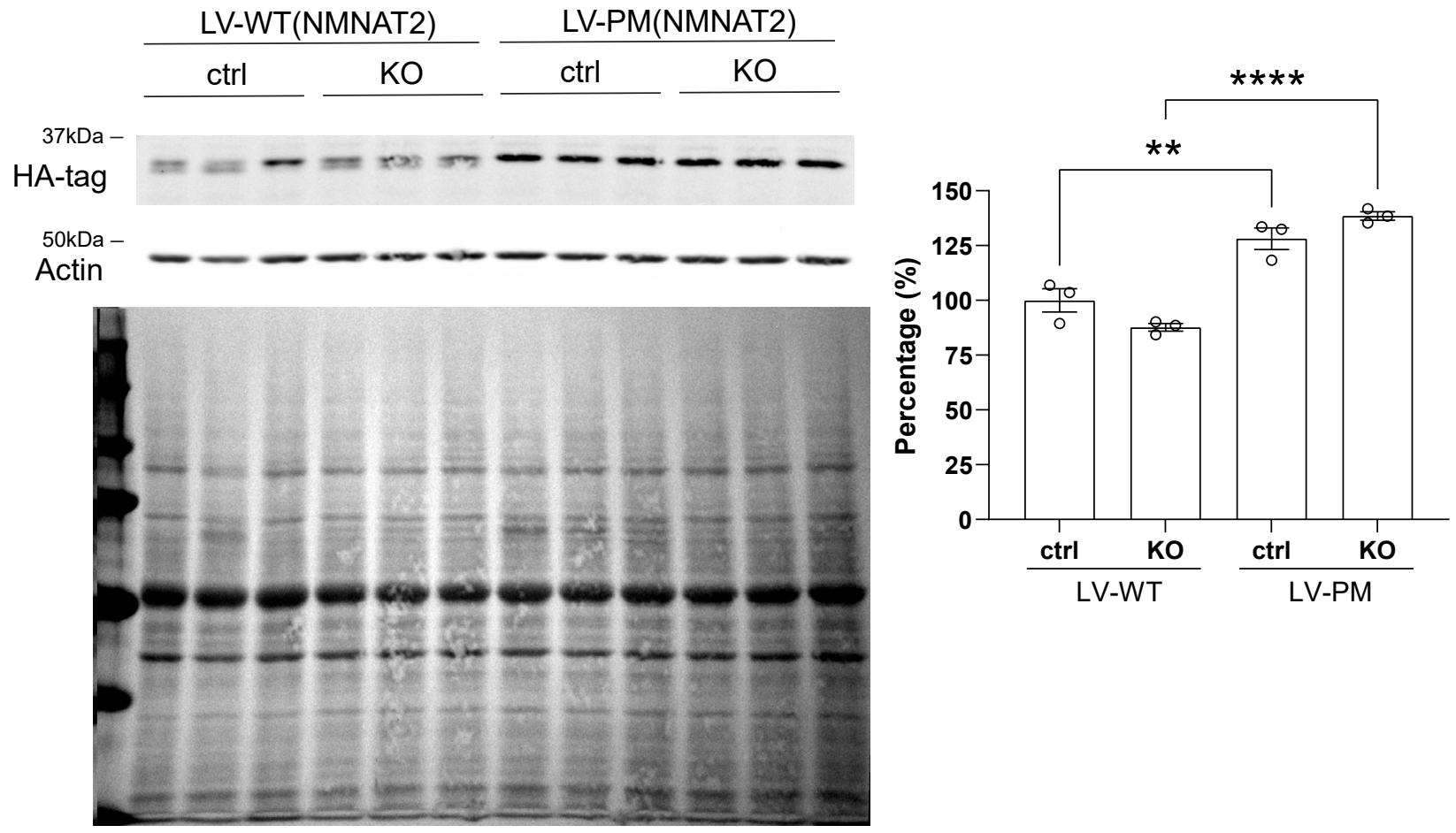

Ponceau Red total protein stain 